Nanoscale Science, ; -13 ginecting and

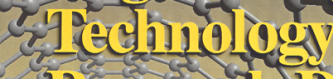

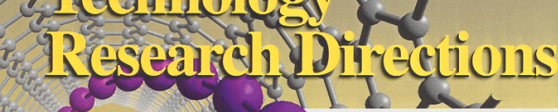

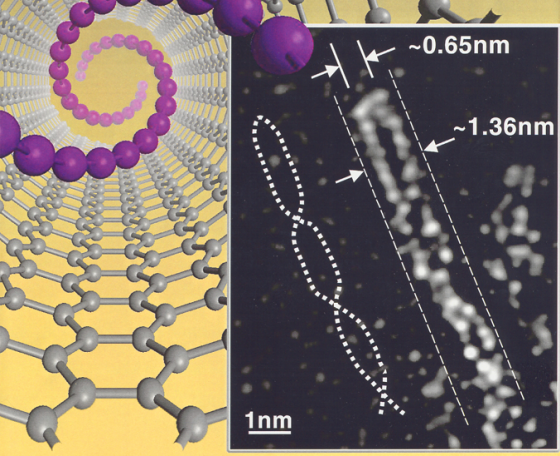




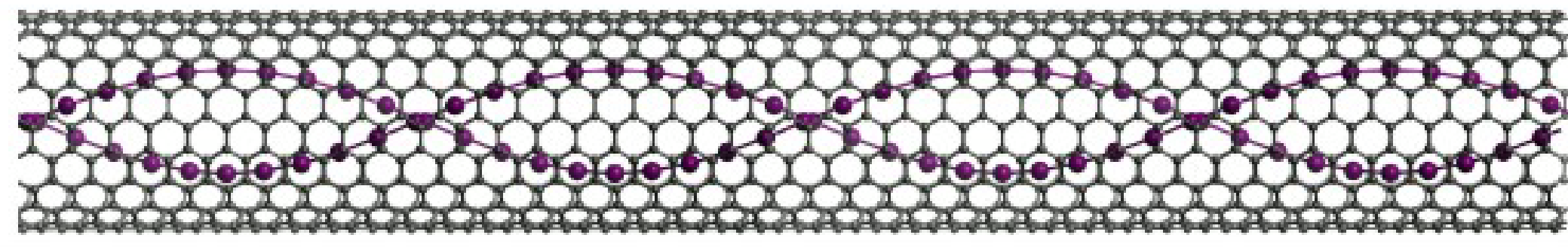

The figure on the front cover shows a Z-contrast transmission electron microscope image of iodine aloms intercalated inside a single wall carbon nanotube in the form of a double helix (X. Fan and S.J. Pennycook, Oak Ridge National Laboratory; E.C. Dickey, P. Ecklund, K. Williams and L. Grigorian, University of Kentucky; R. Buczko and S.T. Pantelides, Vanderbilt University).

This report was prepared at Oak Ridge National Laboratory (ORNL), P.O. Box 2008, Oak Ridge, Tennessee 37831, for the Office of Basic Energy Sciences, U.S. Department of Energy. ORNL is managed by Lockheed Martin Energy Research Corp. for the Department of Energy under Contract No. DE-AC05-960R22464. 


\title{
Nanoscale Science, Engineering and Technology
}

\author{
Research Directions
}

\begin{abstract}
This report describes important future research directions in nanoscale science, engineering and technology. It was prepared in connection with an anticipated national research initiative on nanotechnology for the twenty-first century. The research directions described are not expected to be inclusive but illustrate the wide range of research opportunities and challenges that could be undertaken through the national laboratories and their major national scientific user facilities with the support of universities and industry.
\end{abstract}




\section{TABLE OF CONTENTS}

Basic Energy Sciences Nanoscience/Nanotechnology Group .................................................iii

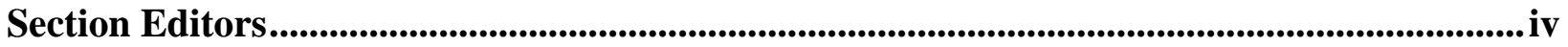

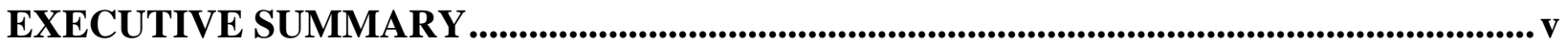

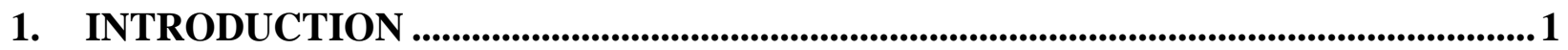

2. EMERGENCE OF PROPERTIES

AT MULTIPLE LENGTH AND TIME SCALES .......................................................... 4

3. MANIPULATION AND COUPLING OF PROPERTIES

AT THE NANOSCALE ........................................................................................................ 10

4. CONTROLLED SYNTHESIS AND PROCESSING AT THE NANOSCALE (PRIMARILY ORGANIC) ...........................................................................................23

5. CONTROLLED SYNTHESIS AND PROCESSING AT THE NANOSCALE (PRIMARILY INORGANIC) ...............................................................................................26

6. NANOSCALE PRECURSORS AND ASSEMBLY

FOR MACROSTRUCTURES AND DEVICES ........................................................ 35

7. UNDERSTANDING AND MIMICKING BIOLOGICAL FUNCTIONS:

“DRAWING INSPIRATION FROM NATURE" ...........................................................4 46

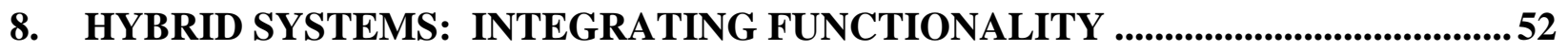

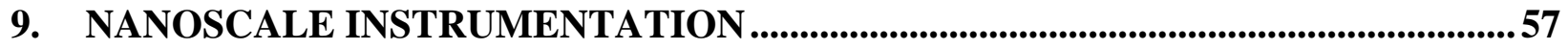

10. INFRASTRUCTURE AND FACILITIES

FOR NANOSCALE SCIENCE AND TECHNOLOGY ...........................................6 67

APPENDIX: NANOMATERIALS RESEARCH CENTERS................................................. 70 


\section{Basic Energy Sciences Nanoscience/Nanotechnology Group}

Chair: Douglas H. Lowndes (ORNL)

A. Paul Alivisatos, Lawrence Berkeley National Laboratory (LBNL)

Mark Alper, Lawrence Berkeley National Laboratory (LBNL)

Robert S. Averback, University of Illinois-Urbana Champaign (UI-UC)

Jacob Barhen, Oak Ridge National Laboratory (ORNL)

Jeffrey A. Eastman, Argonne National Laboratory (ANL)

Dan Imre, Brookhaven National Laboratory (BNL)

Douglas H. Lowndes, Oak Ridge National Laboratory (ORNL)

Ian McNulty, Argonne National Laboratory (ANL)

Terry A. Michalske, Sandia National Laboratories (SNL)

Kai-Ming Ho, Ames Laboratory (AL)

Arthur J. Nozik, National Renewable Energy Laboratory (NREL)

Thomas P. Russell, University of Massachusetts (UM)

Richard A. Valentin, Argonne National Laboratory (ANL)

David O. Welch, Brookhaven National Laboratory (BNL)

NOTE: Information also was provided by university professors and national laboratory research staff. Contributors are listed on the inside back cover page. 


\title{
General Editor
}

\author{
D. H. Lowndes (ORNL)
}

\section{Section Editors}

1. Introduction: D. H. Lowndes (ORNL)

2. Emergence of Properties: D. O. Welch (BNL)

3. Manipulation and Coupling of Properties: I. McNulty (ANL)

4. Controlled Synthesis and Processing (Organic): A. P. Alivisatos (LBNL)

5. Controlled Synthesis and Processing (Inorganic): R. S. Averback (UI-UC)

6. Nanoscale Precursors and Assembly: A. J. Nozik (NREL)

7. Understanding and Mimicking Biological Functions: M. Alper (LBNL)

8. Hybrid Systems: T. A. Michalske (SNL)

9. Nanoscale Instrumentation: J. A. Eastman (ANL)

10. Infrastructure and Facilities: T. P. Russell (UM)

Appendix. Nanomaterials Centers: D. H. Lowndes (ORNL)

NOTE: Jacob Barhen (ORNL) edited contributions from the Engineering Research Program. 


\section{EXECUTIVE SUMMARY}

The principal missions of the Department of Energy (DOE) in Energy, Defense, and Environment will benefit greatly from future developments in nanoscale science, engineering and technology. For example, nanoscale synthesis and assembly methods will result in significant improvements in solar energy conversion; more energy-efficient lighting; stronger, lighter materials that will improve efficiency in transportation; greatly improved chemical and biological sensing; use of low-energy chemical pathways to break down toxic substances for environmental remediation and restoration; and better sensors and controls to increase efficiency in manufacturing.

The DOE's Office of Science has a strong focus on nanoscience discovery, the development of fundamental scientific understanding, and the conversion of these into useful technological solutions. A key challenge in nanoscience is to understand how deliberate tailoring of materials on the nanoscale can lead to novel and enhanced functionalities. The DOE National Laboratories are already making a broad range of contributions in this area. The enhanced properties of nanocrystals for novel catalysts, tailored light emission and propagation, and supercapacitors are being explored, as are hierachical nanocomposite structures for chemical separations, adaptive/responsive behavior and impurity gettering. Nanocrystals and layered structures offer unique opportunities for tailoring the optical, magnetic, electronic, mechanical and chemical properties of materials. The Laboratories are currently synthesizing layered structures for electronics/photonics, novel magnets and surfaces with tailored hardness. This report supplies numerous other examples of new properties and functionalities that can be achieved through nanoscale materials control. These include:

- Nanoscale layered materials that can yield a four-fold increase in the performance of permanent magnets

- Addition of aluminum oxide nanoparticles that converts aluminum metal into a material with wear resistance equal to that of the best bearing steel

- New optical properties achieved by fabricating photonic band gap superlattices to guide and switch optical signals with nearly $100 \%$ transmission, in very compact architectures

- Layered quantum well structures to produce highly efficient, low-power light sources and photovoltaic cells

- Novel optical properties of semiconducting nanocrystals that are used to label and track molecular processes in living cells

- Novel chemical properties of nanocrystals that show promise as photocatalysts to speed the breakdown of toxic wastes

- Meso-porous inorganic hosts with self-assembled organic monolayers that are used to trap and remove heavy metals from the environment

- Meso-porous structures integrated with micromachined components that are used to produce high-sensitivity and highly selective chip-based detectors of chemical warfare agents 
These and other nanostructures are already recognized as likely key components of $21^{\text {st }}$ century optical communications, printing, computing, chemical sensing and energy conversion technologies.

The DOE is well prepared to make major contributions to developing nanoscale scientific understanding, and ultimately nanotechnology, through its materials characterization, synthesis, in situ diagnostic and computing capabilities. The DOE and its National Laboratories maintain a large array of major national user facilities that are ideally suited to nanoscience discovery and to developing a fundamental understanding of nanoscale processes. Synchrotron and neutron sources provide exquisite energy control of radiation sources that are able to probe structure and properties on length scales ranging from Ångstroms to millimeters. Scanning Probe Microscope (SPM) and Electron Microscopy facilities provide unique capabilities for characterizing nanoscale materials and diagnosing processes. DOE also maintains synthesis and prototype manufacturing centers where fundamental and applied research, technology development and prototype fabrication can be pursued simultaneously. Finally, the large computational facilities at the DOE National Laboratories can be key contributors in nanoscience discovery, modeling and understanding.

In order to increase the impact of major DOE facilities on the national nanoscience and technology initiative, it is proposed to establish several new Nanomaterials Research Centers. These Centers are intended to exploit and be associated with existing radiation sources and materials characterization and diagnostic facilities at DOE National Laboratories. Each Center would focus on a different area of nanoscale research, such as materials derived from or inspired by nature; hard and crystalline materials, including the structure of macromolecules; magnetic and soft materials, including polymers and ordered structures in fluids; and nanotechnology integration. The Nanomaterials Research Centers will facilitate interdisciplinary research and provide an environment where students, faculty, industrial researchers and national laboratory staff can work together to rapidly advance nanoscience discovery and its application to nanotechnology. Establishment of these Centers will permit focusing DOE resources on the most important nanoscale science questions and technology needs, and will ensure strong coupling with the national nanoscience initiative. The synergy of these DOE assets in partnership with universities and industry will provide the best opportunity for nanoscience discoveries to be converted rapidly into technological advances that will meet a variety of national needs and enable the United States to reap the benefits of a technological revolution. 


\section{INTRODUCTION}

This report describes important future research directions in nanoscale science, engineering and technology. It was prepared by members of the Office of Basic Energy Sciences (BES) Nanoscience/Nanotechnology (N/N) Group [1] in connection with an anticipated interagency national research initiative titled "Nanotechnology for the Twenty-First Century: Toward a New Industrial Revolution" [2].

The national initiative, described in the report of the Interagency Working Group on Nano Science, Engineering and Technology (IWGN) [2], identifies five "high priority" research areas for additional funding beginning in FY2001. The first and largest of these is "long-term science and engineering research leading to new fundamental understanding and discoveries of phenomena, processes and tools for nanotechnology". The IWGN report identifies the major DOE interest in nanotechnology as basic energy science and engineering. It also mentions several modes of R\&D support that will be needed by DOE/BES to ensure the success of a nano science and technology initiative. Among these are appropriate capital resources for the national labs; secondary funding of universities for collaborations with DOE labs; funding for the national labs to work with other government agencies, and later with industry; and several new national user facilities focused on nanomaterials. The national nanotechnology initiative is considered highly collaborative and interdisciplinary. It is expected to have substantial longterm impact, with the stated intention of facilitating a technological revolution and ensuring that the United States reaps the benefits.

The reason that nanoscale materials and structures are so interesting is that size constraints often produce qualitatively new behavior. We now understand, in a general way, that when the sample size, grain size, or domain size becomes comparable with a specific physical length scale such as the mean free path, the domain size in ferromagnets or ferroelectrics, the coherence length of phonons, or the correlation length of a collective ground state like superconductivity, then the corresponding physical phenomenon will be strongly affected. Although such changes in behavior can be the dominant effects in nanoscale structures, we still have remarkably little experience or intuition for the expected phenomena and their practical implications, except for electronic systems. The physics, chemistry and biology of phenomena occurring in nanoscale systems is effectively a new subject with its own set of physical principles, theoretical descriptions, and experimental techniques, which we are only in the process of discovering. Thus, there is an urgent need for broadly based investigations of the physical phenomena associated with confined systems, especially in materials and structural contexts where the implications are not at all well understood.

Nanotechnology arises from the exploitation of new properties, phenomena, processes, and functionalities that matter exhibits at intermediate sizes between isolated atoms or molecules $(\sim 1$ $\mathrm{nm}$ ) and bulk materials (over $100 \mathrm{~nm}$ ). As opposed to the microscale, the nanoscale is not just another step towards miniaturization, but is a qualitatively new scale. Here quantum and size 
phenomena are allowed to manifest themselves either at a purely quantum level or in a certain "admixture" of quantum and classical components. At the foundation of nanosystems lie the quantum manifestations of matter that become relevant and measurable. Consequently, instead of being a limitation or an elusive frontier, quantum phenomena have become the crucial enabling tool for nanotechnology [3].

This report consists of 10 sections plus an Appendix. Each section deals with a particular nanoscale research theme. The themes were selected by the BES N/N Group as an effective means of organizing and explaining a complex and highly interdisciplinary subject. Thematic organization also permits focusing attention on fundamental ideas with broad implications for nanoscale science, and illuminating the most important opportunities, challenges and barriers to progress, as we seek to understand phenomena and processes on the nanoscale and use this knowledge as the basis for a new nanotechnology.

Most sections of this report follow a similar format. First, a brief summary is given of selected BES and national laboratory capabilities and recent research accomplishments, in order to indicate the current status of research on each scientific theme. Next comes a discussion of the important scientific issues and opportunities that can be addressed through the national laboratories and universities. The principal problems and barriers to progress are identified wherever possible. Finally, future research directions and corresponding research needs are outlined, with emphasis on the promise and potential of certain choices. Thus, this report mainly presents ideas regarding questions to be answered and the kinds of things that could be done by BES in order to take a leadership position in nanoscale science, engineering and technology.

In order to unequivocally identify and characterize nanoscale phenomena it is necessary to directly probe the small samples in which they occur. This is problematic, because sub-micron samples produce tiny signals in most relevant physical measurements. Possible ways to address this problem, by developing new, high sensitivity nanoscale instrumentation, are discussed in section 9 .

BES is especially well prepared for materials, chemical, biological and engineering [4] research on the nanoscale because of its substantial investment in major scientific user facilities, among which are several synchrotron $\mathrm{x}$-ray and high-flux neutron sources. The wavelength or energy range of the radiation provided by these sources is ideally suited for the reciprocal or real space characterization of nanoscopic structures. Their high flux makes possible probing either static nanoscale structure or real-time changes. From many other perspectives the match of synchrotron $\mathrm{x}$-ray and neutron sources to nanoscale science is also ideal, as described in section 10. However, the potential exists for much more effective use of these sources by consolidating and better focusing the nanomaterials research effort. This requires an investment in synthesis and characterization facilities that would be focused on specific nanomaterials research areas and associated with existing major photon and neutron sources, which can be accomplished by establishing several Nanomaterials Research Centers at national laboratories. Establishing such Centers also will facilitate a necessary change in the current infrastructure and scientific mode of operation. The research efforts of scientists who are currently trained in different academic disciplines will be focused into interdisciplinary research groups. The Centers will provide the infrastructure support necessary for these scientists, for large numbers of external users, and for 
the interdisciplinary training of students and postdocs to perform nanoscale research effectively and efficiently. The rationale for establishing Nanomaterials Research Centers is presented in more detail in section 10, and an outline description of possible focal research areas for the Centers is given in the Appendix to this report. These Centers, in association with the principal BES x-ray, neutron and nanofabrication facilities, are expected to be among the most important tools for the national nanotechnology initiative.

\section{References}

1. The members of the BES Nanoscience/Nanotechnology Group are listed in the Preface, and the contributions of some members as section editors for this report also are acknowledged.

2. Nanotechnology for the Twenty-First Century: Toward a New Industrial Revolution, report of the Interagency Working Group on Nano Science, Engineering and Technology (IWGN, chaired by M. Roco, National Science Foundation) to be issued September, 1999.

3. D. N. Noid, R. F. Tuzun and B. G. Sumpter, "On the Importance of Quantum Mechanics for Nanotechnology," Nanotechnology 8, 119 (1997).

4. The relationship of the BES Engineering Research Program (ERP) to nanotechnology follows a path similar to the program's involvement with engineering research for largerscale systems. It is envisioned that eleven topical areas will fall within the programmatic scope of ERP. These areas, along with understanding the quantum relationships and any thermodynamic, chaotic or nonlinear issues that could influence the underlying nanosystems, will capture the important engineering paradigms to be studied and developed under ERP. No programmatic priority is implied in the following list.

- Manufacturing procedures to build tomorrow's nano-machines beyond the current and immediate future silicon-based fabrication technology.

- Property characterization (thermal, mechanical, optical, chemical, biological) at the nanoscale.

- Modeling of the complex behavior of extremely tiny devices at the fundamental molecularmicroscopic-components and assembly levels.

- Innovative control methods for nanosystems.

- Nanoelectronic computing devices.

- Using quantum phenomenology for new communications paradigms.

- Reliable packaging of nano-devices.

- Innovative solutions for energy redistribution, powering and actuation of the nano-machines.

- Micro-instrumentation for on-line diagnostics and control.

- Infrastructure for testing procedures suitable for nanodevices (in a manner analogous to what is now done for IC devices).

- Diagnostics and manipulation of nano-systems for usage, environmental consideration and ability to construct larger systems from the nano-components.

- Friction, lubrication, cracking, failure analysis and nondestructive testing in a nanometer world. 


\section{EMERGENCE OF PROPERTIES AT MULTIPLE LENGTH AND TIME SCALES}

The era of materials technology that we are now entering presents an unprecedented opportunity to understand the emergence of new materials properties on the nanometer scale [1] and to exploit this knowledge for our benefit. The combination of tools responsible for this opportunity includes the ongoing development of synthesis methods that permit atomic and electronic structure to be controlled down to near the atomic scale, in one through three dimensions; the actual or expected availability of instrumental probes that are capable of characterizing nanoscale materials and structures, as well as their properties, on a wide range of length and time scales; and, the continuing explosive growth of computational power which permits theoretical exploration of structure-properties relationships on scales from the atomic to the macroscopic.

Thus far, the most concentrated effort to apply the tools of synthesis, characterization and modeling, in order to understand the consequences of controlling nanoscale structure, has been in studies of the electronic properties of semiconductors and related materials, driven primarily by their vital importance to information technology [2]. Nevertheless, it is quite clear from this experience that many other novel combinations of properties can be expected to emerge, for a wide range of materials, upon entering the world of the nanoscale.

\section{Effects of Size Constraints}

Size constraints alone often produce qualitatively new behavior. For example, if the size of a nanoscale structure becomes less than the characteristic length scale for scattering of electrons or phonons (the mean free path) this can lead to qualitatively new modes of transport for electrical current and/or heat. This effect explains the discovery in the early 1980s of persistent normal (non-superconducting) currents in mesoscale metallic rings [3], and more recently of the ballistic transport of current in carbon nanotubes [4]. Other examples are the remarkably rich set of new phenomena that arise when the de Broglie wavelength of carriers is constrained by the dimensions of quantum dots $[1,2]$.

Thermodynamic properties, including collective phenomena and phase transitions such as ferromagnetism, ferroelectricity, and superconductivity, have long been expected to demonstrate substantial changes when structures contain a small number of the relevant particles or when the system size is comparable to the particle size or the coherence length for collective behavior [5]. Systems with component sizes ranging from a few tenths to about ten nanometers lie at the fuzzy boundary between the quantum and classical domains. Such systems are also in the size range where thermal energy fluctuations and Brownian motion can have significant effects.

Contemporary needs in data storage and information technology have stimulated considerable research activity in nanoscale magnetism [1]. One area presenting great opportunities is the magnetism of complex molecules containing tens to hundreds of atoms with localized magnetic moments. Such "molecular magnets" exhibit many unusual properties including tunneling, quantum coherence, and thermo-induced spin crossover transitions. Possible technological 
applications include the use of magnetic molecules in medicine [6]. Exchange-coupled nanoscale structures also provide a path toward the next generation of powerful permanent magnets [7]. An approach to electronics based on spin, rather than the charge of electron and hole carriers, in nanostructured magnetic devices shows great promise [8]. Ultrathin films (a few atomic layers) yield quasi-two-dimensional magnetic materials, reveal novel magnetic domain physics, and provide a fascinating arena for studying the gradual onset of magnetism [9].

The mechanical properties of materials also change dramatically as the grain size in polycrystalline materials or the concentration of strain fields approaches the nanometer scale [10]. This was demonstrated recently by nanoindentation experiments [11] which showed that atomic-height steps on Au single-crystal surfaces have dramatic effects on the yield strength. Computer simulations suggest significantly higher mechanical strength of grain boundaries in nanocrystalline diamond than those in coarse-grained diamond films [12].

Changes in the strength of nanoscale structural elements, changes in the nature of friction, and changing modes of fluid flow (hence, of lubrication), all will require new design strategies for micromachines. The impact of such changes becomes dramatic as the scale of such machines approaches the nanoscale [13]. Modes of failure also will change, as the scale of devices and machines decreases toward the nanoscale. The causes include different mechanical properties that will modify fracture characteristics; the increased importance of surface tension; and, the enhanced role of diffusion and corrosion at the large surface-to-volume ratios that will occur. Even the notion of the thermodynamic efficiency of a nanoscaled machine or device will differ from its macroscopic, or even microscopic, counterpart because of thermal fluctuations, quantum noise [13] and changes in the efficiency of nanoscaled engines [14]. This is all largely unknown territory.

\section{Shift of Characteristic Time Scales}

This discussion has focussed so far on the emergence of new properties as the characteristic length scale decreases into the nanoscale regime. However, such a length scale change will be accompanied by concomitant changes in the characteristic time scale of physical phenomena. In part, this is no more than the increase in characteristic frequencies that follows from the decreased time required to transit shorter distances at a fixed propagation velocity (for phonons, photons, electrons, etc.). However, genuine changes of regime also will occur. These include an increased importance of surface phonons compared with volume phonons, and ballistic electron transport rather than the scattering-limited transport described by the Boltzmann equation.

Another time-scale phenomenon that will emerge is the increased rate of kinetic processes because of the increased fluctuation rate as the system size decreases and as the reduced dimensionality of important structural features (e.g. surface-to-volume ratio) becomes important or dominant at the nanoscale. The latter effect of course also leads to the increased effectiveness of sensor elements in biological systems. Very large surface-to-volume ratios must at least be taken into account, and possibly exploited, in man-made nanosystems [15]. The shift of characteristic time scales from this variety of mechanisms provides opportunities but will also require the use of instrumentation with improved time resolution and of ultrafast probes. 


\section{Modeling and Simulation}

The emergence of genuinely new phenomena at the nanoscale creates a great need for theory, modeling and large-scale computer simulation in order to understand the new nanoscale phenomena and regime. The links between the electronic, optical, mechanical, and magnetic properties of nanostructures and their size, shape, topology, and composition are not well understood, although for the simplest semiconductor systems, carbon nanotubes, and similar "elementary" systems there has been considerable progress. However, for more complex materials and hybrid structures even the basic outlines of a theory describing these connections remains to be made.

In nanoscale systems, thermal energy fluctuations and quantum fluctuations are comparable to the activation energy scale of the materials and devices, so that statistical and thermodynamic methods must include these effects adequately. Stochastic simulation methods, as well as computational models incorporating quantum and semiclassical methods, are required to evaluate the performance of nanoscale devices. Consequently, computer simulations, both electronic-structure-based and atomistic, will play a major role in understanding materials at the nanometer scale and in the development "by design" of new nanoscale materials and devices. The vastly increased computational capabilities that will become available through the Scientific Simulation Initiative (SSI) will play an important role and will provide many opportunities for substantial progress in this area. Moreover, by exploiting quantum phenomena that have no classical analogues, it is expected that certain computational tasks can be performed much more efficiently than can be done by any classical computer (see the box on the following page).

In addition to such $a b$-initio and large-scale computational methods, there also is a continuing need for phenomenological methods such as Ginzburg-Landau models and mean-field methods to aid in understanding and to develop intuition. There are great opportunities for all scales of theory and modeling in understanding behavior at the nanoscale, but perhaps the greatest challenge and opportunity will be in those transition regions where nanoscale phenomena are just beginning to emerge from the macroscopic and microscale, regimes which are describable by bulk properties plus the effects of interfaces and lattice defects. 


\section{Quantum Computing and Communications at the Nanoscale}

The possibility of transferring quantum information between arbitrarily remote locations (i.e., quantum teleportation [17]) is one of the cornerstones of the emerging field of quantum communications $[18,19]$. Spectacular refinements of the experimental techniques and equipment make it possible for rather modest laboratories to perform "purely quantum" experiments [20] with far reaching epistemological, scientific, and practical consequences [21].

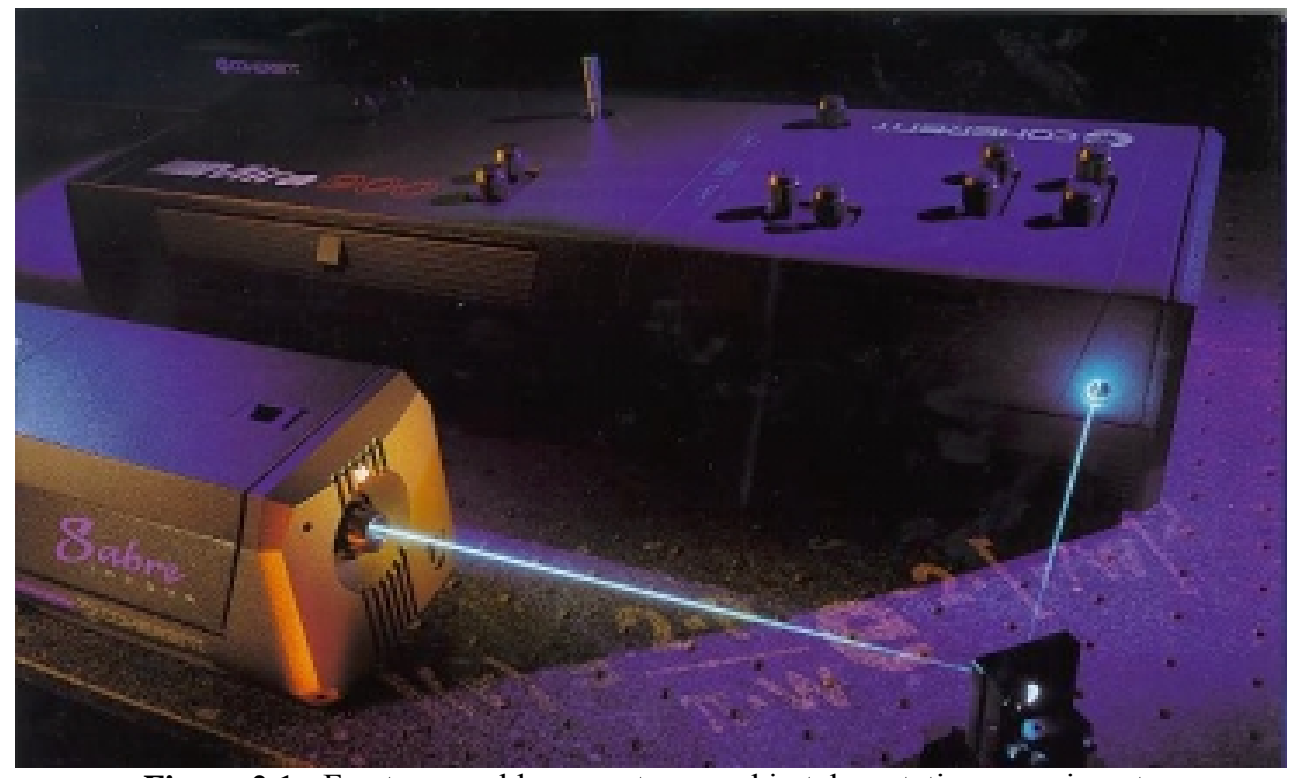

Figure 2.1: Femtosecond laser system used in teleportation experiments

Entanglement is the key to a new realm of quantum phenomena that, until very recently, could not be observed, analyzed, or utilized [22-24]. It is an inherently quantum (i.e. non-classical) property that offers reasonable and consistent explanations to the violation of Bell's inequalities or Schrödinger's famous cat paradox. Quantummechanical analysis yields predictions of non-classical behaviors such as amplitude-phase squeezing or "apparent violation of causality" in experiments involving entangled states. Moreover entanglement offers solid prospects for implementing secure (quantum) cryptography, quantum-computing algorithms, and quantum teleportation.

Measurements performed on such quantum systems would unequivocally demonstrate non-local behavior with unprecedented practical relevance [25]. For instance, if a signal beam (entangled part of the wave function to the 'target') is sent to one person and the idler beam (entangled part to the 'detector'), one person can modulate their own locally received beam and the other can observe the results of the modulation via the visibility parameter. A message sent in this fashion is secure from interception because an eavesdropper lacks the unmodulated beam, which is the 'receiver' beam. If an eavesdropper managed to intercept the unmodulated beam, the person intending to perform the modulation would know it by performing a simple measurement before modulating.

In other applications, intervention on one side cannot be dismissed as a causal influence on the other. In some sense, this implies "faster-than-light" (FTL) communication, where no energy or particles are travelling faster than light, but the wave function is [26]. The dramatic miniaturization in computer technology over the past few decades is rapidly reaching the point at which we will be forced to use quantum physics to describe elementary computational operations [27]. Thus, at the nanoscale the very theory describing what computers can do must be revised.

Initially, researchers in quantum computing tried to understand how the basic operations of a conventional computer might be accomplished using quantum mechanical interactions. However, it was soon realized that quantum physics offered something genuinely new [28]. By exploiting delicate quantum phenomena that have no classical analogues, it is possible to do certain computational tasks much more efficiently [29,30] than can be done by any classical computer. Moreover, these same quantum phenomena allow unprecedented tasks to be performed such as breaking supposedly unbreakable codes, generating true random numbers, and communicating with messages that betray the presence of eavesdropping. In summary, quantum computing and communications are expanding the foundations of information processing in a way that is consistent with quantum physics - the most accurate model of reality that is currently known. 


\section{References}

1. G. Timp, editor, Nanotechnology, AIP Press, Springer-Verlag (New York, 1999).

2. Special Issue on Quantum Dot Structures, Japanese Journal of Applied Physics, Vol. 38, No. 1B (1999).

3. L. L. Sohn, L. P. Kouenhoven, and G. Schoen, editors; Mesoscopic Electron Transport, Kluwer (Dordrecht, 1996).

4. S. Frank, P. Poncharal, Z. L. Wang, and W. A. de Heer, "Carbon Nanotube Quantum Resistors," Science 280, 1744 (1998).

5. Terrell L. Hill, Thermodynamics of Small Systems, Dover (New York, 1994).

6. E. Coronado, P. Delhaes, D. Gatteschi, and J. S. Miller, editors: Molecular Magentism: From Molecular Assemblies to Devices, NATO AISI Series E. Vol. 321, Kluwer (Dordrecht, 1996).

7. D. Givord and M. F. Rossingol, "Coercivity," p. 210, in Rare Earth Permanent Magnets, J. M. D. Coey, editor, Oxford University Press (Oxford, 1997).

8. G. A. Prinz, "Magnetoelectronics," Science 282, 1660 (1998).

9. J. Harris and D. Awschalom, "Thin films squeeze out domains," Physics World 19, (1999).

10. Special Issue on "Mechanical Behavior of Nanostructured Materials," MRS Bulletin, Vol. 24, No. 2, February 1999.

11. T. A. Michalske and J. E. Houston, "Dislocation Nucleation at Nano-Mechanical Contacts," Acta Mater. 46, 391 (1998).

12. P. Keblinski et al., "Amorphous Structure of Grain Boundaries and Grain Junctions in Nanocrystalline Silicon by Molecular-Dynamical Simulation," Acta Mater. 45, 987 (1997); D. Wolf et al., "Phonon-Induced Anomalous Specific Heat of a Nanocrystalline Model Material by Computer Simulation," Phys Rev. Lett. 74, 4686 (1995).

13. J. Knight, “The Dust Mite's Dillema," New Scientist, April 1999, p. 41.

14. J. Travis, "Making Light Work of Brownian Motion," Research News, Science 267, 1593, 1995; B. G. Levi, "Measured steps advance the understanding of molecular motors," Search and Discovery, Physics Today, 19, (1995).

15. R. McGraw and R. LaViolette, "Fluctuations, temperature, and detailed balance in classical nucleation theory," J. Chem. Phys. 102, 8983 (1995).

16. G. Adam and M. Delbrück, "Reduction of Dimensionality in Biological Diffusion Processes," p. 198, in Structural Chemistry and Molecular Biology, A. Rich and N. Davidson, editors, W. H. Freeman (San Francisco, 1968).

17. D. Bouwmeester, J.-W. Pan, K. Mattle, M. Eibi, H. Weinfurter, and A. Zeilinger, "Experimental Quantum Teleportation," Nature 390, 575 (1997).

18. Special Issue: Quantum Communications, J. Mod. Optics 41 (1994).

19. O. Hirota, and C.M. Caves, Quantum Communication, Computing, and Measurement, Plenum Press (1997).

20. W. Tittel, et al., "Long-distance Bell-type tests using energy-time entangled photons," Phys. Rev. A 59, 4150 (1999).

21. A. Watson, "Entangled Trio to Put Nonlocality to the Test," Science 283, 1429 (1999).

22. A. Einstein, B. Podolsky, and N. Rosen, "Can Quantum Mechanical Description of Physical Reality be Considered Complete?" Phys. Rev. 47, 777 (1935).

23. J. S. Bell, "On the Einstein-Podolsky-Rosen Paradox," Phys. 1, 195 (1964); also, "Speakable and Unspeakable in Quantum Mechanics," Cambridge University Press (1988).

24. D. Bouwmeester, et al., "Observation of Three-Photon Greenberger-Horne-Zeilinger Entanglement," Phys. Rev. Lett. 82, 1345 (1999). 
25. T. P. Spiller, "Quantum Information Processing: Cryptography, Computation, and Teleportation," Proc. IEEE 84, 1717 (1996).

26. D. M. Greenberger, "If one could build a macroscopic Schrodinger Cat state, one could communicate superluminally," Physica Scripta T 76, 57 (1998).

27. R. Feynman, "Quantum Mechanical Computers," Optics News 11, 11 (1985).

28. A. Steane, "Quantum Computing," Rept. Prog. Phys. 61, 117 (1998).

29. H. K. Lo, S. Popescu, and T. Spiller, "Introduction to Quantum Computation and Information," World Scientific (1998).

30. G. Berman, et al., "Introduction to Quantum Computers," World Scientific (1998). 


\section{MANIPULATION AND COUPLING OF PROPERTIES AT THE NANOSCALE}

\section{A. Current Status}

\section{Semiconductor Properties}

Research on the optical, electronic, and transport properties of semiconductors at the nanoscale is experiencing astonishing growth. The fundamental properties of nanoscale semiconductor structures can be dramatically altered by controlling their size and shape without changing their composition. When the electrons and holes in semiconductors are confined to dimensions less than their de Bröglie wavelength (typically 1-30 nm) quantum mechanical size effects appear. The carrier confinement can be in one dimension (quantum films or quantum wells), two dimensions (quantum wires), or three dimensions (quantum dots, QDs, or nanocrystals). The most dramatically affected electronic properties include: (a) a large blue-shift of the absorption edge with decreasing size, which can be several electron-volts for semiconductor nanocrystals, resulting in absorption or photoluminescence that can be tuned to range from near-UV through the visible to the near IR; (b) conversion of optical spectra from continuous bands to discrete lines; (c) concentration of oscillator strengths in fewer allowed transitions, resulting in greatly enhanced non-linear optical properties; (d) enhanced photoredox and photocatalytic properties due to higher redox potentials of photogenerated electrons and holes; (e) conversion of direct bandgap semiconductors to indirect bandgap semiconductors and vice versa; (f) slowed relaxation and cooling of photogenerated hot carriers, resulting in reduced electron-phonon interactions; (g) greatly enhanced excitonic transitions at room temperature; (h) an optical absorption coefficient that increases with the inverse-cube of the nanocrystal radius.

In the newly-coined field of "nano-optics" the wavefunction of a QD is tailored for a specific response. Electronic and optical properties of QDs have been observed that closely resemble those of simple atomic systems, including sharp line spectra and relatively simple nonlinear optical signatures [1]. In recent experiments employing wavefunction engineering techniques, the excitonic wave function in a single QD was manipulated and monitored on a time scale short compared to the loss of quantum coherence, by controlling the phase and polarization of twopicosecond optical pulses [2]. These experiments have extended the concept of coherent control in semiconductor nanostructures to the limit of a single quantum system in a zero-dimensional QD, and provide the basic tools to create more complex wavefunctions in future such "solid state atoms".

\section{Ballistic Electron Devices}

One of the challenges of electron physics is to create devices that allow controlled charge and energy transport at nanometer length scales. Such nanoscale quantum devices may be components in electrical circuits or they could provide sources of stimulated coherent radiation. They might be fabricated from semiconductor wafers, clusters of atoms, carbon fibers, or even from biological molecules. Ballistic electron devices comprise one class of quantum devices that hold great promise for future communications applications. The size and energy at which they 
operate directly determines their characteristic switching times. For example, present-day nanoscale electron waveguides are typically fabricated from semiconductor materials, operate at very low temperatures, and typically have picosecond switching times. The recent discovery of conduction quantization in carbon nanotubes [3,4] provides hope that such devices might eventually be fabricated to operate with far higher switching speeds at room temperature.

The dynamics of ballistic electron propagation can be studied using the two-dimensional electron gas (2DEG) that can be created at the interface of two semiconductor materials such as GaAs and AlGaAs. Electron channels and cavities of variable shape can be formed at such an interface by means of gates placed on the outer surface of the semiconductor wafer. The observation [5] that electron conductance is quantized for electron currents passing between point contacts separated by approximately $100 \mathrm{~nm}$ showed conclusively that the wave nature of electrons plays a critical role at the nanoscale. At temperatures of $1 \mathrm{~K}$ or lower, the electronphonon interaction can be neglected and the electron motion becomes ballistic. The shape of the confining walls in the two-dimensional plane can be controlled and strongly influences the waveguide conductance [6].

Much of the research effort on electron waveguides until now has focused on the effect of quantum chaos on conductance, both in the presence and absence of external magnetic fields. Waveguide cavities used in 2DEG experiments typically have widths and lengths of order 100$1000 \mathrm{~nm}$. In this regime, enough propagating modes are present that semiclassical methods (such as periodic orbit theory) can be used to make predictions about the electron conductance. Numerical and some laboratory work [6] has shown that the electron conductance has qualitatively different behavior depending on whether the waveguide cavities give rise to integrable or chaotic behavior. One of the important discoveries in quantum chaos research is that if a quantum chaotic system has a classical counterpart that is fully chaotic, then it exhibits universal statistical behavior in qualitative agreement with the predictions of random matrix theory [7]. Recently, a possible connection has been made between these predictions for the effects of chaos and disorder in electron waveguides. However, as the size of electron waveguides becomes closer to the nanoscale we enter the quantum regime, where far less is known about the electron dynamics.

One reason for the need to understand the quantum influence on conductance is the possibility of developing more energy-efficient and much higher density electronic circuitry at the nanoscale. It has been shown that integrable waveguides may have better electron-trapping ability than chaotic waveguides [8]. It appears that the conductance in integrable systems can be shut down and electrons stored for longer periods. Waveguides formed at semiconductor interfaces (by means of gates on the outer surface) have leads and cavities which are soft, and the dynamics of these systems is more integrable than chaotic. Indeed, recent work on the conductance of softwall waveguides with mixed dynamics has shown fractal fluctuations in the conductance as a weak magnetic field is varied [9]. Resonances give rise to waveguide capacitance in the presence of high-frequency electromagnetic driving fields, allowing enhanced power absorption [10]. An important problem about which little is currently known is the effect that Coulomb interactions between electrons have on the electron conductance in the fully quantum regime. A related development, based on the nonlinear charge distribution in capacitively coupled conductors, is the possibility of quantum scanning capacitance microscopy at the nanoscale [11]. 


\section{Magnetic Properties}

Dramatic quantization and other effects also occur in magnetism and magnetic materials at the nanoscale. The ability to control thin-film growth at the near-atomic level to form epitaxial and heteroepitaxial structures has recently been extended to magnetic nanostructures, including metallic, oxide, and semiconducting phases [12,13]. This progress is driven both by the intriguing physics of these materials and by the $\$ 50 \mathrm{~B}$ per year magnetic storage industry $(\$ 150 \mathrm{~B}$ per year if magnetic recording tape, video, etc. are included). Materials advances have opened up entirely new avenues of study. To illustrate, theoretical limits suggest that a near doubling of the energy product of hard magnets may be possible with an optimum nanoscale configuration of $\mathrm{Fe}-\mathrm{Co}, \mathrm{Nd}-\mathrm{Fe}-\mathrm{B}$, or $\mathrm{Nd}-\mathrm{Fe}-\mathrm{Al}$ bulk metallic glass $[14,15]$. However, putting this on firm footing requires a better understanding of coercivity development and domain wall pinning in nanocomposite hard magnets, how coercivity is developed in bulk metallic glasses, and extensive microstructural evaluation to determine the makeup and locations of atomic clusters that contribute to the magnetic remanence.

\section{Thermal Properties}

Thermal transport properties of nanostructured materials have received relatively little attention in the past decade. It is well known that polycrystalline materials exhibit lower thermal conductivity than low-defect single crystals of the same material. Several investigators realized recently that this could result in significantly reduced thermal conductivities in nanostructured materials such as yttria-stabilized zirconia (YSZ), which could lead to improvements for applications such as thermal barrier coatings [16,17]. Reduced thermal conductivities are expected because of a reduction in the phonon mean free path due to grain boundary scattering. Indeed, recent research has determined that the thermal conductivity is reduced approximately two-fold at room temperature in 10-nm grain-sized YSZ, compared to coarse-grained or single crystal YSZ [18]. While several groups are now investigating the thermal properties of nanostructured materials, there is currently no detailed understanding of nanostructure-thermal properties relationships. Unexplored at present, but perhaps even more interesting, is the effect of grain size on the thermal properties of high-conductivity phonon-based conductors such as diamond.

In contrast to the reduced thermal conductivity expected for nanostructured thin films or coatings, opportunities exist for increasing thermal transport rates in fluids by suspending nanocrystalline particles in them. These "nanofluids" have recently been shown to exhibit substantially increased thermal conductivities and heat transfer rates compared to fluids that do not contain suspended particles [19]. However, there is again no real understanding of the mechanisms by which nanoparticles alter thermal transport in liquids. Multibillion-dollar industries, including transportation, energy, electronics, textiles, and paper, employ heat exchangers that require fluids for efficient heat transfer. If researchers can improve these fluids, then manufacturers can make heat exchange systems smaller and lighter, and they can reduce the amount of pumping energy and heat-transfer fluid required.

\section{Energy Conversion and Transport}

Energy conversion and transport in nanostructures impacts a variety of fields and applications. Although energy conversion and transport at macroscales is relatively well understood, it is not 
clear how it occurs at the nanoscale. For example, it is well known that thermoelectric refrigerators and engines are not as efficient as other energy conversion devices because heat conduction by phonons is too high in thermoelectric materials. There is partial and currently controversial evidence that nanostructuring can both improve electron transport [21-23] and reduce phonon transport [24-27]. However, it is not clear how the phonon transport is actually reduced. Similarly, the mechanisms for friction, and for the control of friction, at the nanoscale are not understood (see the box following "Future Research Directions" below).

\section{Photonic Crystals}

Photonic crystals have emerged as unique structures with the capability to manipulate the flow of light energy as well as its coupling with excited electronic states. Many novel applications of these structures have been proposed in the areas of optoelectronics and photocatalysis [28-31]. To create photonic crystals operating at optical wavelengths the smallest feature sizes must be of the order of $100 \mathrm{~nm}$, clearly in the realm of nanotechnology. State-of-the-art semiconductor technology $[32,33]$ has recently been used to fabricate three-dimensional photonic-bandgap crystals at $1.5 \mu \mathrm{m}$ and $10 \mu \mathrm{m}$ wavelengths, using stacks of microfabricated polysilicon bars. Further development of lithography techniques (such as two-photon methods) at smaller length scales will be needed to extend this technology to visible wavelengths.

There has been rapid progress recently in the development of alternative approaches [34] to fabricate photonic crystals by using self-assembled systems that order spontaneously [35] at nanoscale dimensions. Organic or inorganic spheres or block-polymers have been used as the starting material. The photonic crystals fabricated by such techniques consist of porous air cavities in an interconnected dielectric background. The wavelength of the photonic band gap is controlled by both the spatial periodicity and properties of the dielectric crystal. Further development in self-assembly techniques should allow assembling these structures into integrated device systems at low cost [36].

Great advantages are expected from these structures, including the control of radiative properties and lifetimes that in turn can control chemical reactions and catalysis. One novel phenomenon is the localization of light in a disordered photonic crystal, in analogy to the localization of electrons in disordered systems [37]. Such photonic-crystal structures have immense potential

for a large variety of optoelectronics devices. Another remarkable recent development with interesting potential applications is the demonstration of organic photonic-crystal lasers [38]. 


\section{Optical Matter}

Developments in the nascent field of "optical matter" have demonstrated the production of highly organized, multidimensional states of combined matter and light fields [39]. By using crossed laser beams to create carefully controlled standing wave patterns, microscopic structures such as polystyrene spheres or more recently, various alkali atoms, can be trapped in the resulting arrays of potential wells to form "optical crystals" [40-42]. The methods employed are an outgrowth of the optical molasses techniques developed for ultrahigh precision atomic spectroscopy at micro-Kelvin temperatures [43]. Optical crystals have demonstrated unusual binding, coupling, and energy-quantization properties, and they similarly offer possibilities for precision spectroscopy.

Optical matter has been considered by T. Hänsch and others to be an entirely new state with inherently different properties than conventional states of matter [40-42]. To date, opticalmatter experiments have utilized only visible-light wavelengths. However, the groundwork has been laid for extension of the methods and theory to the nanometer length scale. The principal means to achieve this would be by preparation of intense coherent $\mathrm{x}$-ray standing-wave fields using perfect-crystal optics. The precursors for this technology have already been used for surface structure determination [44] and have been proposed for photo-assisted materials growth at nanometer length scales [45]. The anomalous propagation of on-resonance standing-wave fields in crystals (Borrmann effect) may also find application. [46].

\section{Role of Theory and Simulation}

The optical, electronic, magnetic and mechanical properties of nanostructures depend sensitively on their size, shape, composition and outer-shell structure. The links between these properties and degrees of freedom are incompletely understood but are crucial to their eventual technological application. Establishing this link is the main challenge for theory in the field of nanostructures.

Quantum confinement provides a means to control the optical properties of nanostructures via their size, but different shapes can dramatically alter nanostructure properties even at constant size. Composition (e.g., $\operatorname{In}_{\mathrm{x}} \mathrm{Ga}_{1-\mathrm{x}} \mathrm{As}$ ) changes the confinement potential and the interfacial strain, thus also altering the properties. The outer-shell structure determines how much of the wave function is contained in the nanostructure and how much "spills out", which also can affect the electronic properties. Size, shape, composition, and outer-shell structure are controlled during growth or subsequent processing of the nanostructure. For example, one can grow spherical or pyramidal InAs dots via colloidal and Stranski-Krastanov methods, and the outer shell can be organic (e.g., TOPO) or inorganic (GaAs). Sizes can be controlled via colloidal chemistry. Similar variability in size, shape, composition and outer-shell structure is afforded by many other growth methods. 
The current status of nanostructure theory is as follows:

(a) The theory of electronic and optical properties includes the effective-mass theory and its $\underline{k} \cdot \underline{p}$ generalization, tight-binding, and full-pseudopotential methods. These approaches are able to treat the link between size and shape and the nanostructure optical and electronic properties with various levels of precision and accuracy. Recent applications include InP, CdSe, and InAs free-standing and embedded QDs.

(b) The theory of magnetism and spin in nanostructures is in its infancy. Most theories do not yet treat the spin variable and the magnetic moments.

(c) The theory of thermodynamics and stability in nanostructures is also incomplete. Methods are available for small nanostructures (less than 100 atoms), for example the local density approximation, but the methods known for larger systems are of lower quality.

\section{B. Key Scientifc Questions}

Many questions remain unanswered concerning the coupling of properties to our advantage at the nanoscale. For instance, we are pressed to inquire:

(a) How do QDs couple electronically when formed into arrays and how does order versus disorder affect the coupling? Does the electronic structure change once the QDs are spatially organized, e.g. vertically versus laterally? What is the maximum possible inter-dot conductivity? Can "minibands" be formed analogous to those that are formed in quantumfilm superlattices?

(b) How does the ability of a nanostructure to accept carriers affect its properties ("Coulomb blockade")? Is carrier transport between QDs characterized by hopping, and can good carrier transport (e.g. photoconductivity and photovoltaic effects) be obtained?

(c) Can magnetism be instilled in a non-magnetic material via quantum size effects, and what controls the spin lifetime?

(d) How does size quantization affect the kinetics of photoinduced electron transfer between semiconductors and molecules?

(e) Can a QD of an indirect-gap material ( $\mathrm{Si}, \mathrm{Ge}, \mathrm{GaP}$ ) become optically direct?

(f) Can useful non-linear optical devices for optical computing, information storage and transport be achieved with QDs or other nanostructured materials?

(g) Can phonon transport be reduced by using nanostructured superlattice materials, and can nanostructuring improve the thermoelectric figure of merit by improving electron transport?

Closely related materials questions that must be addressed include:

(a) How does the shape of the nanostructure affect its properties?

(b) How are the photocatalytic properties affected?

(c) Are there surface states in nanostructures, and what is the effect of the surface structure on their properties?

(d) Can one alter the optical response of a nanostructure by changing its outer-shell structure (e.g. by passivation)?

(e) Is the solubility of dopants in nanostructures reduced or enhanced relative to the bulk? Can QDs be doped? 
Many of the above questions can be addressed convincingly using theoretical tools that are available now or under construction. This will establish a direct link with experiments and open the way for nanostructured design of targeted properties. One immediate hurdle is to develop theoretical tools to describe accurately the electronic structure of large QDs $\left(10^{4}-10^{6}\right.$ atoms).

Ballistic electron devices, in particular, test the limits of theoretical knowledge because they involve small systems where boundary conditions may play a dominant role and the wave nature of the electron is of fundamental importance. In these systems the interplay of electric and magnetic interactions is not yet fully understood. The devices themselves generally are open systems involving electron-decay processes and tunneling phenomena. Multi-photon or multiphonon interactions also may be important. Their dynamics may be chaotic or regular, or may involve a mixture of chaotic and resonant behavior. The mechanisms governing electron transport in 2DEGs still are not completely understood. At temperatures of the order of $1 \mathrm{~K}$ the interaction of electrons with phonons is negligible. However, the electrons, whose motion is largely phase-coherent, still experience long-range Coulomb interactions, random electric and magnetic fields due to impurities, and may exhibit manifestations of chaos due to boundary effects. The electrons are thus sensitive to their local environment, which depends critically on the fabrication methods and materials used. Electronic conduction at low temperatures is also affected by resonances determined by the shape of the waveguide channels and cavities, as well as the materials used. Ballistic electron devices operate at the interface between quantum mechanics and thermodynamics, two disciplines that have not yet been reconciled when dealing with small numbers of particles. An understanding of all of these effects in the fully quantum regime is essential, if we are to understand electrical circuits that function at the nanometer scale.

Since many properties of nanodevices are related to quasibound states of electrons, it is also important to understand their dynamics. A case in point is the decaying states of electron waveguides. For waveguides with long straight leads and a rectangular cavity, the states with a continuous spectrum in the leads correspond to the continuous field states, while the quasibound states of the cavity correspond to unstable states. The case where a discrete energy of the bare states in the cavity is close to the minimum energy of the continuous spectrum of the leads results in interesting nonlocal phenomena associated with "super-luminality." Since nonlocality is a quantum characteristic, this situation also can provide an interesting experimental test of fundamental problems in quantum mechanics. Moreover, electron waveguides can provide an experimental measurement of the magnitude of the "dressing" of the excited unstable state. This has a bearing on the decoherence problem as it relates to nanoscale quantum computers because quantum computation is reliable only for time scales during which one can neglect decoherence.

\section{Future Research Directions}

The future applications of nanoscale devices are both exciting and boundless. The greatest nearterm payoff is likely to be the development of ultrahigh-speed and efficient electronic, optical, and magnetic devices for communications, switching, computing, and storage, and their 


\section{Friction and Control of Friction at the Nanoscale}

Nano-devices and the new ultra small technology are expected to significantly improve the performance of robots, computers, communication, and other electro/optical/mechanical devices. However, friction imposes a significant limitation in usage of these tiny devices. As a manifestation of the nano in the macro, hundreds of millions of dollars will be lost as a result of wear, friction, breakdowns, and wasted energy. Research to control friction at the nanoscale will result in highly improved performance in the macro world and can produce significant economic savings [47].

Traditional lubrication involves the use of organic substances whose functional groups can adsorb onto polar surfaces to form a closed-packed arrangement of almost perpendicularly oriented lubricant chains. However, nano-machine lubricant selection is complicated by new considerations. Due to the built-in-place nature of nano-mechanics, lubrication by the conventional means of hidden and contacting surfaces is prohibitive. Fluid lubricants may also introduce capillary and viscous shear mechanisms, which result in energy dissipation. Research will span the following topics:

Mechanisms for Friction and Lubrication at the Nano-Scale. Despite great progress made through the past half century, many basic issues in fundamental tribology such as origin of friction and failure of lubrication have remained unsolved. Moreover, current reliable knowledge related to friction and lubrication is mainly applicable to macroscopic systems and machinery and, most likely, will be of only limited use (if at all) in nano-systems. When the thickness of the lubrication film is of the same order as the molecular or atomic size, the behavior of the lubricant becomes significantly different [48]. Understanding the mechanisms of friction, lubrication, and other interfacial phenomena at atomic and molecular scales will provide designers and engineers the required tools and capabilities to control and monitor friction, reduce unnecessary wear, and predict mechanical faults and failure of lubrication in nano-devices $[49,50]$.

Electromechanical and Optical Control of Friction. Friction can be manipulated by applying small adjustments (perturbations) to accessible elements and parameters of the sliding system [51]. This requires apriori knowledge of the strength and timing of the perturbations, similar in concept to the "residual statics" perturbation problem in seismic imaging, where global optimization techniques have shown great promise [52]. In addition, surface roughness and thermal noise are significant factors in making decisions about control on the micro and nano-scales. Research to study the response of the sliding system and lubricating thin films to small mechanical, electrical, and optical excitations will result in identifying better ways to control friction and to create a novel type of electromechanical lubricant at the nano-scale.

Control of Chaos in Friction. In many situations, the behavior of the lubricant and the sliding surfaces is chaotic. Thus control of chaos and targeting it toward desired behaviors is an important issue for lubrication and friction. Techniques for controlling chaos have been under systematic investigation since 1990 [53]. The main issue is to develop efficient and robust methods to selectively drive low and high dimensional chaotic dynamics by using tiny external perturbations [54]. Both feedback and non-feedback techniques have to be adjusted and applied to control chaos in friction [55]. Control of chaos in spatially extended systems has not yet reached a high applicability level. Thus, research in this direction is very significant.

Quantum Control of Friction. When the size of sliding surfaces decreases to the nano-scale, quantum effects play an important role. Quantum control of friction is significantly different in concept and methods from classical control, and is much less understood. The main difficulties associated with developing efficient quantum control techniques are: (i) insufficient understanding of many-particle systems; (ii) lack of exact solutions for large many-body systems; (iii) inaccessibilty/collapse under measurements of the state function; and (iv) lack of robust algorithms for two-point boundary value problems. A focused effort to develop algorithms for quantum control of friction is required.

Computational Aspects. Research on friction and lubrication is highly interdisciplinary and probes the very foundations of physics, chemistry, mathematics, mechanical, electrical and chemical engineering, and computer science. Due to the high cost of experiments, computational modeling and simulations [56] to support and predict experimental findings will play a significant role. Developing new, accurate, fast, and stable methods to solve large systems of nonlinear differential equations will be one of the crucial issues in research on friction at the nanoscale. 
incorporation into highly integrated and massively parallel systems. Current trends toward everincreasing circuit complexity, miniaturization, and reduced power consumption in the information technology arena can be expected to accelerate, and the drive to develop and commercialize these nanotechnologies will become paramount. But related developments that are further afield at present, such as quantum computing and cryptography, will also profit from a comprehensive and sustained nanoscience research program.

Many other examples can be found to illustrate the close link between fundamental studies of nanoscale phenomena and their technological applications. Consider interlayer magnetic coupling in multilayer structures. Although initial studies of magnetic coupling in multilayers were motivated primarily by scientific curiosity, as giant magnetoresistance (GMR) and the concomitant phenomenon of oscillatory interlayer magnetic coupling were discovered, the technological relevance of these discoveries resulted in products within an extraordinarily short ten-year period. Future developments that can be anticipated include nonlinear magneto-optical effects in magnetic multilayers and nanostructures that may lead to a new generation of magnetic sensors, recording media, and imaging methods.

Another area which can be expected to benefit significantly from the development of nanostructures exhibiting novel geometries and optical coupling is inexpensive mass-production of high-resolution optical components. "Optical matter" methods may prove instrumental to organize and assemble them on the nanoscale. These devices will be capable of focusing and guiding not only microwave and visible light, but shorter wavelengths from the ultraviolet down to the $\mathrm{x}$-ray region. Diffraction-limited performance at the nanoscale requires nanometer wavelengths, i.e. $x$-rays. Such $x$-ray optical elements operating at unprecedented $(<10 \mathrm{~nm})$ spatial resolution can in turn be used to fabricate other nanostructures, quantum devices, and integrated devices, and to form intense $\mathrm{x}$-ray nanoprobes for microscopy, diffraction, and scattering experiments. Large arrays of nanofocusing $\mathrm{x}$-ray optics will quickly find applications in nanolithography, combinatorial materials and combinatorial biology analysis systems.

Nanoscale coupling of properties clearly has significant energy-related implications as well. For example, nanocrystalline semiconductors in the form of fractal films of particles, isolated colloidal QDs, ordered and disordered arrays of close-packed colloidal QDs, and two-and threedimensional arrays of self-organized epitaxially grown QDs have many potential and existing applications in renewable energy systems. These include very inexpensive and color tunable (from clear to colored to black) photovoltaic solar cells based on the dye-sensitization of nanocrystalline wide bandgap oxides $\left(\mathrm{like}^{\mathrm{TiO}}{ }_{2}\right)$ operating in a photoelectrochemical cell, and novel solar cells with extremely high conversion efficiency. If slow cooling of photogenerated hot-carriers and hot carrier transport in nanostructured electrodes can be achieved, it may be possible to develop highly efficient hot-carrier solar cells based on QD arrays. Nanocrystals could also be used as efficient photocatalysts for photodetoxification of polluted or toxic water and air streams. Semiconductor nanocrystals and nanostructures may be used as efficient photoactive materials for solar-photon conversion of simple molecules to fuels and chemicals, for instance photolytic water splitting to produce hydrogen, photoreduction of carbon dioxide to alcohol and hydrocarbon fuels, and photoreduction of molecular nitrogen to ammonia for fertilizer production. 
From another standpoint, a deeper understanding of the physics of phonon transport in nanostructured materials may facilitate production of practical all-solid-state and environmentally clean thermoelectric energy-conversion devices with performances far superior to current vapor-based refrigerators and combustion-based engines. The pervasive role of hard and soft magnets in electric-power production and utilization is another arena in which new nanoscale magnetic materials may yield substantial energy savings by reducing losses and conserving natural resources consumed in the generation and use of electricity.

Lastly, carbon-based nanotubes have the potential to act as a hydrogen storage medium that could exhibit very high storage density per unit weight, which is critical for hydrogen-based transportation systems. A crucial issue is whether or not the hydrogen could be extracted efficiently from such a storage medium at relatively low temperatures.

\section{References}

1. D. Gammon, E. S. Snow, B. V. Shanabrook, D. S. Katzer, and D. Park, "Fine structure splitting in the optical spectra of single GaAs quantum dots," Phys. Rev. Lett. 76, 3005 (1996).

2. N. H. Bonadeo, G. Chen, D. Gammon, D. S. Katzer, D. Park, and D. G. Steel, "Nonlinear nano-optics: probing one exciton at a time," Phys. Rev. Lett. 81, 2759 (1998); N. H. Bonadeo, J. Erland, D. Gammon, D. Park, D. S. Katzer, and D. G. Steel, "Coherent optical control of the quantum state of a single quantum dot," Science 282, 1473 (1998).

3. S. Frank, P. Poncharal, Z. L. Wang, and W. A. de Heer, "Carbon nanotube quantum resistors," Science 280, 1744 (1998).

4. J. K. Gimzewski and C. Joachim, "Nanoscale science of single moleculoes using local probes," Science 283, 1683 (1999).

5. B. J. van Wees, L. P. Kouwenhoven, E. M. M. Willems, C. J. P. M. Harmans, J. E. Mooij, H. van Houten, C. W. J. Beenaakker, J. G. Williamson, and C. T. Foxon, "Quantum ballistic and adiabatic electron transport studied with quantum point contacts," Phys. Rev. B43, 12431 (1991).

6. C. M. Marcus, A. J. Rimberg, R. M. Westervelt, P. F. Hopkins, and A. C. Gossard, "Conductance fluctuations and chaotic scattering in ballistic microstructures," Phys. Rev. Lett. 69, 506 (1992); I. H. Chan, R. M. Clarke, C. M. Marcus, K. Campman, and A. C. Gossard, "Ballistic conductance fluctuations in shape space," Phys. Rev. Lett. 74, 3876 (1995); Y. Wang, J. Wang, H. Guo, and C. Roland, "Tunnelling through quantum-dot systems: a study of the magneto-conductance fluctuations," J. Phys. Condens. Matter 6, L143 (1994).

7. H. U. Baranger and P. A. Mello, "Mesoscopic transport through chaotic cavities: a random S-matrix theory approach," Phys. Rev. Lett. 73, 142 (1994); C. H. Lewenkopf and H. A. Weidenmuller, "Stochastic versus semiclassical approach to quantum chaotic scattering," Ann. Physics 212, 53 (1991); P. Seba, K. Zyczkowski, and J. Zakrzewski, "Statistical properties of random scattering matrices," Phys. Rev. E54, 2438 (1996).

8. G. B. Akguc and L. E. Reichl, "Statistical properties of the scattering matrix of a chaotic electron waveguide," submitted to Phys. Rev. E.

9. R. Ketzmerick, "Fractal conductance fluctuations in generic chaotic cavities," Phys. Rev. B54, 10841 (1996); A. S. Sachrajda, R. Ketzmerick, C. Gould, Y. Feng, P. J. Kelly, 
A. Delage, and Z. Wasilewski, "Fractal conductance fluctuations in a soft-wall stadium and a sinai billiard," Phys. Rev. Lett. 80, 1948 (1998).

10. K. Na and L. E. Reichl, "Effect of the quasibound states on quantum transport in a ballistic electron waveguide," Phys. Rev. B59, 13073 (1999).

11. B. G. Wang, X. Zhao, J. Wang, and H. Guo, "Nonlinear quantum capacitance," Appl. Phys. Lett. 74, 2887 (1999).

12. E. E. Fullerton, J. S. Jiang, C. H. Sowers, J. E. Pearson, and S. D. Bader, "Structure and magnetic properties of exchange-spring Sm-Co/Co superlattices," Appl. Phys. Lett. 72, 380 (1998).

13. R. Notzel, "Self-organization of nanostructures on planar and patterned high-index semiconductor surfaces," Microelectronics Journal 28, 875 (1997).

14. J. Kuma, N. Kitajima, Y. Kanai, and H. Fukunaga, "Maximum energy product of isotropic Nd-Fe-B-based nanocomposite magnets," J. Appl. Phys. 83, 6623 (1998).

15. A. Inoue, A. Takeuchi, and T. Zhang, "Ferromagnetic bulk amorphous alloys," Metall. Mater. Trans. A29, 1779 (1998).

16. S. Raghavan, H. Wang, R. B. Dinwiddie, W. D. Porter, and M.J. Mayo, "The effect of grain size, porosity and yttria content on the thermal conductivity of nanocrystalline zirconia," Scripta Mater. 39, 1119 (1998).

17. P. G. Klemens, in: Chemistry and Physics of Nanostructures and Related Non-Equilibrium Materials, (TMS, Warrendale PA), p. 97-104 (1997).

18. J. A. Eastman, U. S. Choi, S. Li, G. Soyez, L. J. Thompson, and R. J. DiMelfi, in: Proceedings of the International Symposium on Metastable, Mechanically Alloyed, and Nanocrystalline Materials (ISMANAM'98), in press.

19. S. Lee, U.S. Choi, S. Li, and J. A. Eastman, J. Heat Transfer, 121, 280-289 (1999).

20. L. D. Hicks and M. S. Dresselhaus, "Effect of Quantum-Well Structure on the Thermoelectric Figure-of-Merit," Phys. Rev. B47, 12727 (1993).

21. L. D. Hicks and M. S. Dresselhaus, "Thermoelectric figure-of-merit of a one-dimensional conductor,"Phys. Rev. B47, 16631 (1993).

22. L. D. Hicks, T. C. Herman, and M. S. Dresselhaus, "Use of quantum-well superlattices to obtain a high figure-of-merit from nonconventional thermoelectric materials," Appl. Phys. Lett. 63, 3230 (1993).

23. L. D. Hicks, T. C. Herman, X. Sun, and M. S. Dresselhaus, "Experimental study of the effect of quantum-well structures on the thermoelectric figure of merit," Phys. Rev. B53, 10493 (1996).

24. S.M. Lee, D. G. Cahill, and R. Venkatasubramanian, "Thermal conductivity of Si-Ge superlattices," Appl. Phys. Lett. 70, 2957 (1997).

25. G. Chen, "Size and interface effects on thermal conductivity of superlattices and periodic thin-film structures," J. Heat Transfer 119, 220 (1997).

26. G. Chen and M. Neagu, "Thermal conductivity and heat transfer in superlattices," Appl. Phys. Lett. 71, 2761 (1997).

27. G. Chen, "Thermal conductivity and ballistic-phonon transport in the cross-plane direction of superlattices," Phys. Rev. B57, 14958 (1998).

28. E. Yablonovitch, "Inhibited spontaenous emission in solid-state physics and electronics," Phys. Rev. Lett. 58, 2059 (1987).

29. S. John, "Strong localization of photons in certain disordered dielectric superlattices," Phys. Rev. Lett. 58, 2486 (1987). 
30. C. M. Soukoulis, ed., "Photonic band gap materials," Proceedings of NATO Advanced Study Institute (Kluwer, 1996).

31. J. D. Joannopoulos, R. D. Meade, and J. N. Winn, "Photonic Crystals: Molding the flow of light," Princeton University Press (1995); J. D. Joannopoulos, P. R. Villeneuve, and S. H. Fan, "Photonic crystals: putting a new twist on light," Nature 386, 143 (1997).

32. "Photonic crystals," Physics Today , p. 17 (January 1999).

33. J. G. Fleming and S.-Y. Lin, "A three-dimensional photonic crystal with a stop band between 1.35 and $1.95 \mu$ m," Optics Lett. 24, 49 (1999); S.-Y. Lin, J. G. Fleming, D. L. Hetherington, B. K. Smith, R. Biswas, K. M. Ho, M. M. Sigalas, W. Zubrzycki, S. R. Kurtz, J. Bur, "A three-dimensional photonic crystal operating at infrared wavelengths," Nature 394, 251 (1998).

34. "Photonic crystals: whole lotta holes," Chem. Engr. News, p. 38, (23 November 1998).

35. J. E. G.J. Wijnhoven and W. L. Vos, "Preparation of photonic crystals made of air spheres in titania," Science 281, 802 (1998); A. A. Zakhidov, R. H. Baughman, Z. Iqbal, C. X. Cui, I. Khayrullin, S. O. Dantas, I. Marti, and V. G. Ralchenko, "Carbon structures with threedimensional periodicity at optical wavelengths," Science 282, 897 (1998); A. Imhof and D. J. Pine, "Ordered macroporous materials by emulsion templating," Nature 389, 948 (1997); O. D. Velev, T. A. Jede, R. F. Lobo, and A. M. Lenhoff, "Porous silica via colloidal crystallization," Nature 389, 448 (1997); B. T. Holland, C. F. Blanford, and A. Stein, "Synthesis of macroporous minerals with highly ordered three-dimensional arrays of spheroidal voids," Science 281, 538 (1998).

36. Y. N. Xia and G. M. Whitesides, "Soft lithography," Angewandte Chemie 37, 551 (1998).

37. See, for example: K. N. Vlasov, "Different regimes of light localization in a disordered photonic crystal," abstract SC21.09, presented at Am. Phys. Soc. Centennial Meeting (Atlanta GA, March 1999).

38. M. Meier, A. Mekis, A. Dodabalapur, A. Timko, R. E. Slusher, J. D. Joannopoulos, and O. Nalamasu, "Laser action from two-dimensional distributed feedback in photonic crystals," Appl. Phys. Lett. 74, 7 (1999); M. Berggren, A. Dodabalapur, R. E. Slusher, Z. Bao, A. Timko, and O. Nalamasu, "Organic lasers based on lithographically defined photonic-bandgap resonators," Eletron. Lett. 34, 90 (1998).

39. M. M. Burns, J.-M. Fournier, and J. A. Golovchenko, "Optical matter: crystallization and binding in intense optical fields," Science 249, 749 (1990).

40. A. Hemmerich and T. W. Hänsch, "Two-dimesional atomic crystal bound by light," Phys. Rev. Lett. 70, 410 (1993).

41. A. Hemmerich, C. Zimmerman, and T. Hänsch, "Sub-kHz Rayleigh resonance in a cubic atomic crystal," Europhys. Lett. 22, 89 (1993).

42. "Three-dimensional optical molasses binds a new type of crystal," p. 17 in Physics Today (June 1993).

43. P. Verkerk, B. Lounis, C. Salomon, C. Cohen-Tannoudji, J.-Y. Courtois, and G. Grynberg, "Dynamics and spatial order of cold cesium atoms in a periodic optical potential," Phys. Rev. Lett. 68, 3861 (1992); P. S. Jessen, C. Gerz, P. D. Lett, W. D. Phillips, S. L. Rolston, R. J. C. Spreeuw, and C. I. Westbrook, "Observation of quantized motion of Rb atoms in an optical field," Phys. Rev. Lett. 69, 49 (1992).

44. P. Fenter, F. Schreiber, L. Berman, G. Scoles, P. Eisenberger, and M.J. Bedzyk, "On the structure and evolution of the buried S/Au interface in self-assembled monolayers: $\mathrm{x}$-ray standing wave results," Surf. Sci. 413, 213 (1999). 
45. J. Li, A. Mirabedini, L. J. Mawst, D. E. Savage, R. J. Matyi, and T. F. Kuech, "Effect of interface roughness on performance of $\mathrm{AlGaAs} / \mathrm{InGaAs} / \mathrm{GaAs}$ resonanttunneling diodes," $J$. Cryst. Growth 195, 617 (1998).

46. H. Batelaan, E. M. Rasel, M. K. Oberthaler, J. Schmiedmayer, and A. Zeilinger, "Anomalous transmission in atom optics," J. Mod. Opt. 4, 2629 (1997).

47. Y. Braiman, V. Protopopescu, and J. Barhen, "Friction and Control of Friction in Thin Films at the Nanoscale: Towards Novel Forms of Lubrication," (in preparation, January 1999).

48. Yuan-Zhong Hu, S. Granick, "Microscopic Study of Thin Film Lubrication and its Contribution to Macroscopic Tribology," Tribology Letters 5, 81 (1998).

49. Y. Braiman, F. Family, H. G. E. Hentschel, "Nonlinear Friction in the Periodic Stick-Slip Motion of Coupled Oscillators," Phys. Rev. B 55, 5491 (1997).

50. J. Shimizu, H. Eda, M. Yoritsune, E. Ohmura, "Molecular Dynamics Simulation Of Friction on the Atomic Scale," Nanotechnology 9118 (1998).

51. M. Heuberger, C. Drummond, and J. Israelachvili, "Coupling of Normal and Transverse Motion during Frictional Sliding," Journal of Physical Chemistry B 102, 5038 (1998).

52. J. Barhen, V. Protopopescu and D. Reister, "TRUST: A Deterministic Algorithm for Global Optimization," Science 276, 1094 (1997).

53. E. Ott, C. Grebogy. J. A. Yorke, “Controlling Chaos," Phys. Rev. Lett. 64, 1196 (1990).

54. Y. Braiman, I. Goldhersch, "Taming Chaotic Dynamics by Weak Periodic Perturbations," Phys. Rev. Lett. 66, 2545 (1991).

55. K. Pyragas, "Continuos Control of Chaos by Self-controlling Feedback," Phys. Lett. A 170, 421 (1992).

56. B. Bhushan, J. Israelachvili, and U. Landman, "Nanotribology: Friction, Wear, and Lubrication at the Atomic Scale," Nature 374, 607 (1995). 


\section{CONTROLLED SYNTHESIS AND PROCESSING AT THE NANOSCALE (PRIMARILY ORGANIC)}

\section{A. Current Status}

An important new field of chemistry and materials science is emerging, dedicated to the systematic and parallel patterning of matter on the nanometer scale [1]. The controlled positioning of atoms within small molecules is of course routinely achieved by chemical synthesis of moles of identical molecules. Nanometer-size objects are much larger entities, with thousands or even millions of atoms in them. There are many powerful new approaches to patterning on the nanoscale that are fundamentally serial in nature, for instance atom manipulation using scanning probe tips, or electron beam lithography. These are tremendously important techniques. However, there is also a pressing need to develop methods to prepare macroscopic quantities of nanoscale components in complex, designed patterns, using techniques of self assembly. The success of this endeavor will allow the fundamental discoveries of nanoscale science to find wide application in many fields, and especially in energy related technologies. This field is developing rapidly, and this section includes just a brief survey of recent accomplishments.

\section{Accomplishments in the Synthesis of Individual Building Blocks}

Nanocrystals. In the last decade there have been significant advances in the preparation of nanocrystals [2,3]. Many common materials, such as metals, semiconductors and magnets can be prepared as nanocrystals, using colloidal chemistry techniques. The concepts of ligand exchange and surface derivitization have been well developed, and these methods permit nanocrystals with narrow size distribution (typically $5-15 \%$ variation in diameter) to be isolated and then used further as chemical reagents. This field has been aided greatly by the improved understanding of size-dependent scaling laws, which have emerged from fundamental studies in chemical physics and condensed matter physics. The fact that a simple property like light emission depends so strongly upon the particle size in semiconductors has greatly facilitated the development of reliable preparations. The same size dependence has also led to a wide range of applications in unexpected areas, such as in biological tagging $[4,5]$.

Nanotubes and Rods. The exciting discovery of the fullerenes was followed closely by the discovery of nanotubes of carbon [6]. Nanotubes show tremendous promise as building blocks for new materials. Because of their topology, nanotubes have no dangling bonds, and so despite being very small, they do not exhibit "surface effects." As a consequence, individual nanotubes exhibit nearly ideal electrical, optical, and mechanical properties. Nanorods are also under extensive development and investigation.

Polymeric Electronic Materials: Dendrimers and Block Copolymers. The last decade has seen tremendous advances in the preparation of organic building blocks of considerable complexity [7-9]. The discovery of a new topology for polymers, dendrimers, has led to an exciting new class of nanoscale components with interesting optical and mechanical properties. There have 
also been steady advances in engineering new phases using block copolymers; for instance, the recent development of tri-component blocks is noteworthy in this regard. While it is not, strictly speaking, a nanotechnology the tremendous advances in organic electronics, such as organic light emitting diodes, must be noted, since that field is more than likely to benefit by advances in organic nanoscale synthesis in the future.

\section{Templated Growth and Assembly}

Mesoporous Inorganic Solids. In the last decade, great advances have occurred in the preparation of mesoporous inorganic solids [10]. The initial work showed that it is possible to use organic surfactant molecules to prepare a complex pattern. That pattern can serve as the template for the formation of an inorganic phase. This has led to many exciting discoveries in chemical synthesis, and to immediate practical advances in catalysis.

In the design of complex materials, such as electrical devices, we currently rely on the ability to lithographically create designed patterns. In the last decade, nanoscale objects, such as nanocrystals, have been assembled into periodic arrays, or supercrystals. Such arrays exhibit novel optical and electrical characteristics. Several proposals have been put forward for how to pattern nanocrystals and nanotubes using biological molecules [11-13].

\section{Relation to Energy Science}

Many fundamental phenomena in energy science, such as electron transfer and exciton diffusion, occur on the nanometer length scale. Thus, the ability to inexpensively pattern and process matter on this length scale can be an important asset in designing new-generation photovoltaic cells and batteries. The potential power of this idea is clearly illustrated by the Graetzel photovoltaic cell, which is almost randomly assembled from nanoscale components, and yet very cheaply achieves $10 \%$ power efficiency. Isolated nanoscale components also exhibit nearly ideal mechanical properties, and the consequences of this for new materials design have yet to be fully exploited.

\section{B. Key Questions}

Many fundamental scientific issues are unanswered in this field. Among them are:

- What is the largest inorganic or organic cluster that can be prepared in the laboratory as a molecule, with a precise number of atoms in it?

- What are the surface reconstructions and rearrangements of atoms in nanocrystals and nanorods? Is it possible to prepare epitaxial core-shell systems in nanocrystals?

- Can single wall carbon (or other) nanotubes of a single length and helicity be purified as isolated molecular species? Is it possible to reproducibly prepare heterojunctions from one-dimensional nanostructures?

- Is it possible to use parallel self-assembly techniques to control the relative arrangements of nanoscale components according to a complex, designed sequence? 
- Can chemical synthesis be used to generate materials with complex, macroscopic shapes and desirable mechanical properties?

\section{Future Research Directions}

The conventional distinctions between soft and hard materials, or between inorganic and organic materials are rapidly disappearing. Nanometer-size organic and inorganic building blocks are being developed rapidly. The ability to assemble these building blocks into complex, hybrid materials with designed properties is opening the door to a new generation of composite materials. Over the next twenty years, these new classes of materials can transform energyrelated technologies, including catalysis, photovoltaics and batteries.

\section{References}

1. A. P. Alivisatos, P. F. Barbara, A. W. Castleman, J. Chang, D. A. Dixon, M. L. Klein, G. L. McLendon, J. S. Miller, M. A. Ratner, P. J. Rossky, S. I. Stupp, M. E. Thompson, "From molecules to materials: Current trends and future directions," Advanced Materials 10, 1297-1336 (1998).

2. L. Brus, "Semiconductor colloids: Individual nanocrystals, opals and porous silicon," Current Opinion in Colloid \& Interface Science 273, 199-241 (1996).

3. T. P. Martin, "Shells of atoms," Physics Reports-Review Section of Physics Letters 273, 199-241 (1996).

4. W. C. W. Chan, S. N. Nie, "Quantum dot bioconjugates for ultrasensitive nonisotopic detection," Science 281, 2016-2018 (1998).

5. M. Bruchez, M. Moronne, P. Gin, S. Weiss, A. P. Alivisatos, "Semiconductor nanocrystals as fluorescent biological labels," Science 281, 2013-2016 (1998).

6. M. Terrones, W. K. Hsu, H. W. Kroto, D. R. M. Walton, Nanotubes: A revolution in materials science and electronics, Vol. 199, pp 189-234 (1999).

7. O. A. Matthews, A. N. Shipway, J. F. Stoddart, "Dendrimers - Branching out from curiosities into new technologies," Progress in Polymer Science 23, 1-56 (1998).

8. S. I. Stupp, V. LeBonheur, K. Walker, L. S. Li, K. E. Huggins, M. Keser, A. Amstutz, "Supramolecular materials: Self-organized nanostructures," Science 276, 384-389 (1997).

9. D. A. Tomalia, "Starburst Cascade Dendrimers - Fundamental Building-Blocks for a New Nanoscopic Chemistry Set," Advanced Materials 6, 529-539 (1994).

10. D. M. Antonelli, J. Y. Ying, "Mesoporous materials," Current Opinion in Colloid \& Interface Science 1, 523-529 (1996).

11. R. C. Mucic, J. J. Storhoff, C. A. Mirkin, R. L. Letsinger, "DNA-directed synthesis of vinary nanoparticle network materials," Journal of the American Chemical Society 120, 12674-12675 (1998).

12. A. P. Alivisatos, K. P. Johnsson, X. G. Peng, T. E. Wilson, C. J. Loweth, M. P. Bruchez, P. G. Schultz, "Organization of Nanocrystal Molecules Using DNA," Nature 382, 609-611 (1996).

13. E. Braun, Y. Eichen, U. Sivan, G. BenYoseph, "DNA-templated assembly and electrode attachment of a conducting silver wire," Nature 391, 775-778 (1998). 


\section{CONTROLLED SYNTHESIS AND PROCESSING AT THE NANOSCALE (PRIMARILY INORGANIC)}

\section{A. Current Status and Accomplishments}

Scientific and technological breakthroughs in materials research and development are often directly coupled to progress in synthesis and processing. Nanoscience and nanotechnology currently are following this path. Three basic strategies are presently widely employed for producing nanostructured materials: the production of nanoparticles and their delivery onto surfaces; the growth of epitaxial films and surface layers; and the fabrication of ultra-fine-grain monolithic and composite bulk materials.

The growth of epitaxial thin films, in combination with lithography and ion implantation, is currently the most advanced and productive route in this field. Such films are employed for a variety of applications, including Josephson junctions, quantum dots, optical devices, etc. They provide model structures for testing ideas about the properties of nanostructured materials. Although much effort is already focused on epitaxial growth, this is a huge area and many new breakthroughs can be anticipated. In contrast, the processing of bulk nanostructured materials has developed far more slowly, despite their promise for use in a wide range of structural and magnetic applications. The main processing schemes include the direct consolidation of nanoparticles, the sintering of powders that consist of micron-sized particles which are themselves rendered nanocrystalline by mechanical working, and phase transformations, mostly of amorphous alloys. These processes, however, are subject to problems associated with porosity and grain growth [1], which will require fundamental studies on the kinetic behaviors and mechanical properties of these systems to resolve.

The synthesis of nanoparticles also is a large and mature area of research, which has been driven in the past largely by the need for improved catalysts. However, the development of in situ diagnostics and characterization for studying the synthesis of nanoparticles in real time offers new opportunities to tailor the properties of nanoparticles for a wide variety of other specific functions.

\section{B. Key Questions, Research Opportunities and Barriers to Progress}

\section{Science of Epitaxial Growth}

The science of epitaxial growth is the foundation for a major part of future nanotechnology. At present, investigations of growth are well funded only for a few specific materials where technical applications are clearly identified. The examples of silicon-germanium research and the growth of high temperature superconductors illustrate an interesting contrast for the future development of this field. Superconducting materials are sufficiently complicated that development of a science of epitaxial growth is a slow process, often characterized by specific prescriptions for each material. Si-Ge research deals with a much simpler system and in contrast has been wonderfully productive of new concepts relating to fundamental step edge behavior, 
surface morphological structures and strain-induced metastable topologies, for example. Most importantly, it has revealed the effect of these structures on the growth of perfect and nanostructured thin films [2]. What requires recognition is that different types of materials exhibit different classes of behavior, so that knowledge of $\mathrm{Si}-\mathrm{Ge}$ fails to illuminate the science that determines growth processes for other classes of materials. To understand the different behaviors will require a greatly increased commitment to basic research on epitaxy and surface topology for a wide variety of prototype materials (such as simple ionic materials, model metals and organic deposits on inorganic substrates), as well as studies of growth-enhancement methods such as ion and laser-beam assisted deposition.

An area of film growth deserving special attention is the deposition of nanoparticles and clusters, which are useful for creating a variety of surface features. Most important is the ability to control the size, size distribution and organization of the nanoparticles. For example, postprocessing of nanoparticles deposited on patterned near-nanostructured materials either by heating or by using directed beams of photons or ions, can lead to patterned nanostructures, as recently illustrated by the alignment of quantum dots [3]. Another potential processing method to reduce the average cluster size and the size distribution, and provide for particle alignment, employs Coulomb repulsion in the growth process [4]. A first test of this concept utilizes buffer layer assisted growth in which atoms are deposited on top of an inert-gas buffer layer condensed on a cold substrate, in order to make the clusters highly mobile and allow them to self-assemble into an ordered array, but without coalescence. In this test, a significant reduction in the average size and size distribution of Fe clusters deposited on $\mathrm{Cu}(111)$ was observed [5], as described in the box on the next page.

It is evident that studies of growth mechanisms and surface topology at the atomic level can provide a scientific base of understanding from which the synthesis and nanostructuring of a wide variety of technically important materials (many of which remain to be identified) can be effectively undertaken. This will require parallel funding for independent research teams focused on different materials systems, and a commitment to provide state-of-the-art instrumentation by which epitaxial materials can be created and explored at the atomic level.

Progress in this field will require that both synthesis equipment and advanced surface characterization systems such as STM, AFM, LEEM, etc. be made much more widely available to research teams. Support for in situ investigations using central facilities such as UHV transmission electron microscopy and synchrotron radiation, with deposition capabilities included, will also be necessary. Existing electron microscopy centers and synchrotron laboratories provide a convenient means to make these facilities available to a larger number of users. 


\section{Self-Assembled Growth of Quantum Dots with Coulomb Repulsion}

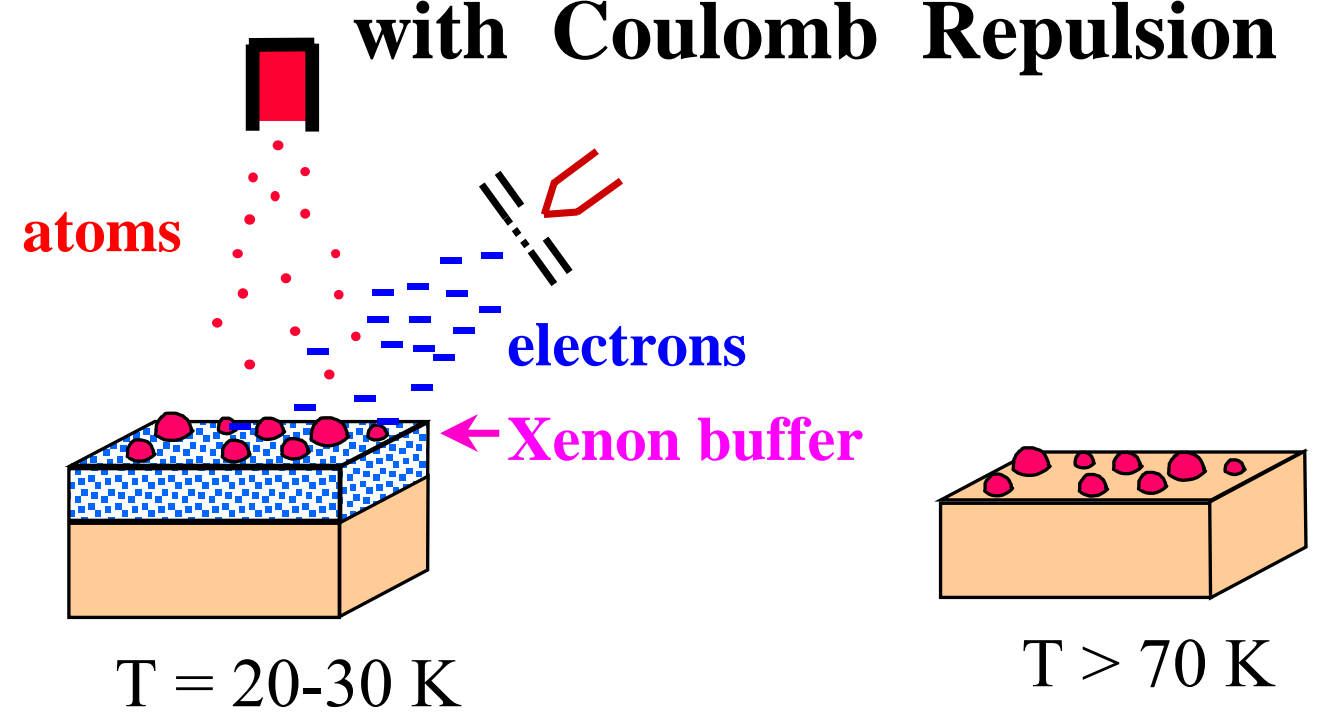

By introducing Coulomb repulsion during the growth of quantum dots (QDs) it should be possible to achieve smaller dots with a more uniform size and spacing. To test this idea Fe clusters were deposited on a solid Xe buffer layer (200 ML) on $\mathrm{Cu}(111)$ at low temperature. The Xe buffer layer provides a high-mobility surface for QD (nanocluster) diffusion and isolates the growing QDs from the substrate. By charging the 3D QDs with electrons, their Coulomb repulsion inhibits cluster aggregation resulting in smaller QDs with a more uniform size. Any combination of QD and substrate materials may be created with this highly parallel growth technique, if only the clusters can be charged and the substrate cooled.

\section{NO ELECTRONS}

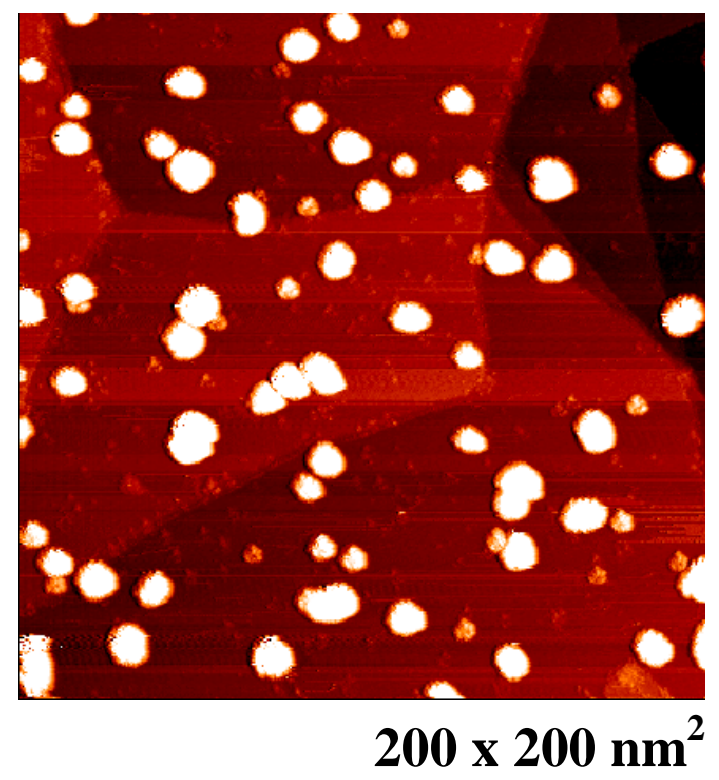

\section{WITH ELECTRONS}

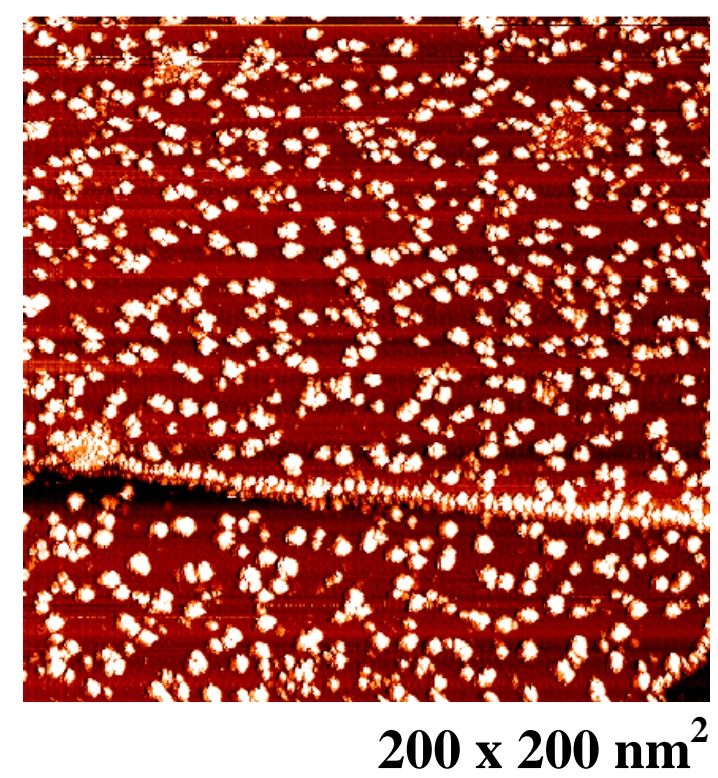




\section{Driven Systems}

A very promising area for processing three-dimensional bulk nanoscale structures originates with the recent discovery that mesoscopic structures can be obtained by nonequilibrium processing, such as ion irradiation, implantation, and mechanical working (ball milling, etc.) [68]. Contrary to equilibrium situations where precipitates should always coarsen to reduce interfacial energy, competition between dynamical processes operating during processing may result in the stabilization of multiphase (composite) materials, where the different phases coexist at a mesoscopic, tunable length scale. An example is illustrated in Fig. 5.1, where results of computer simulation on the ball milling process are shown. The system represents two immiscible metals subjected to shear deformation at elevated temperatures where limited diffusion takes place. Control of the length scale by varying the two control parameters, shearing rate and temperature, is clearly observed. Interesting new properties have been found and others predicted when this coexistence of phases takes place at a nanometric scale. Nanocomposites of hard and soft magnetic phases, for example, make it possible to fabricate materials with both large magnetic anisotropy and large saturation magnetization [9]; nanocomposites of elastically hard and soft metals provide great strength to materials; and metal nanoparticles in glass offer non-linear optical properties [10].
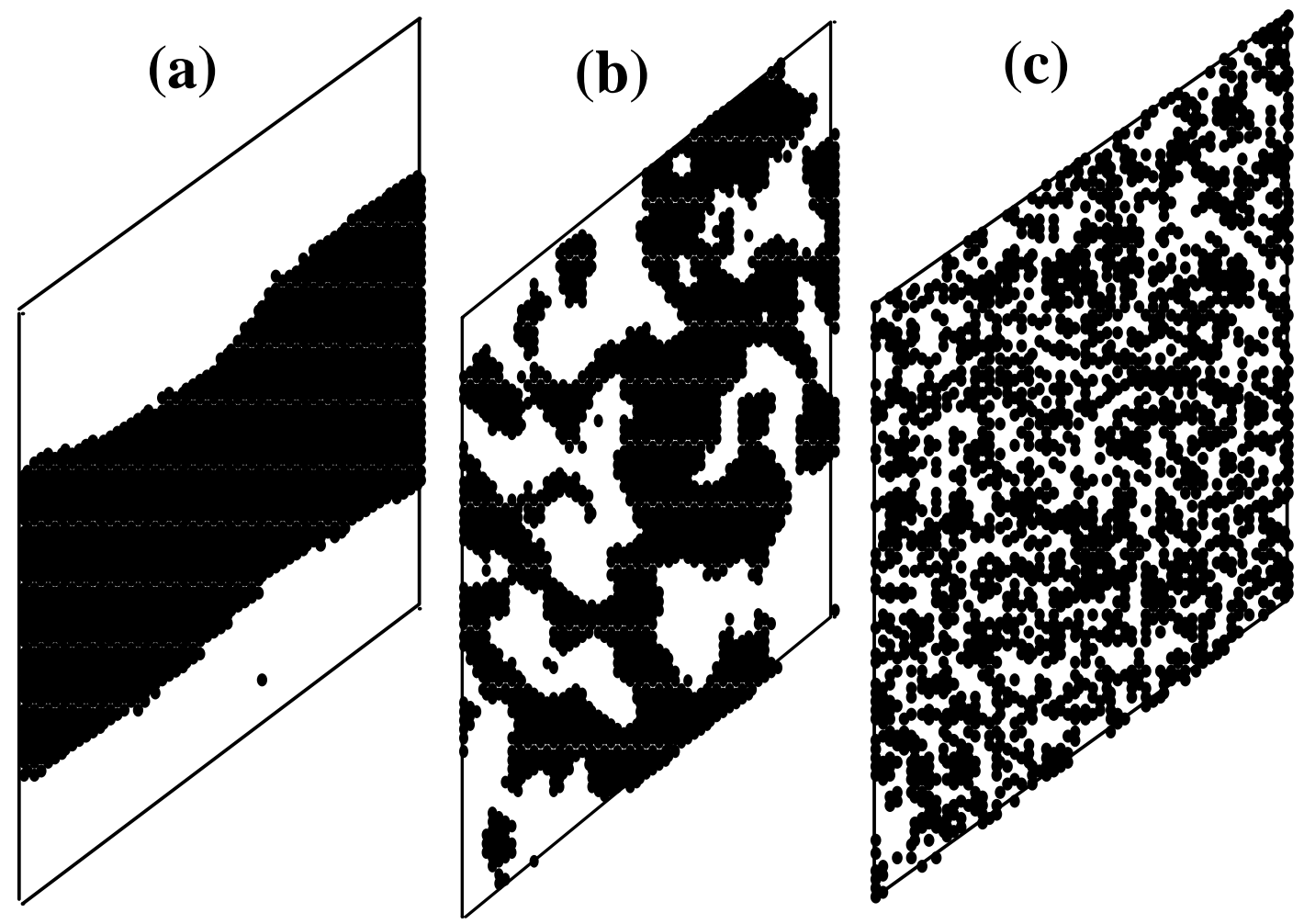

Figure 5.1: Typical steady-state microstructures as observed in a (111) plane at different shearing rates, $\gamma_{\mathrm{sh}}$ : (a) $\gamma_{\mathrm{sh}}=1 \mathrm{~s}^{-1}$; (b) $\gamma_{\mathrm{sh}}=10^{3} \mathrm{~s}^{-1}$; (c) $\gamma_{\mathrm{sh}}=10^{5} \mathrm{~s}^{-1}$. The initial state is a $\mathrm{A} / \mathrm{B}$ bilayer, and the temperature is held constant at $\mathrm{T}=400 \mathrm{~K}$; only $\mathrm{B}$ atoms are shown [6]. 
Before non-equilibrium processing such as this can be employed to full advantage in creating mesoscopic structures, it will be necessary to understand the effect of processing parameters on the final microstructures in a number of situations. This is a challenging task because the theory of driven systems is not well developed in real materials. This calls for combined efforts in modeling, computer simulation, and critical testing. Ion irradiation can play an important role in this context, since the elemental processes occurring during irradiation are very well known. Indeed some work along these lines has been carried out. Roughening of superlattices for improved GMR properties [11], ion mixing and implantation of metal layers in $\mathrm{SiO}_{2}$ for optical devices [12], and ripple formation during high temperature sputtering illustrate these ideas [13]. The information obtained from irradiation studies will be of great benefit for understanding the more complex situations, such as mechanical working, since the underlying science for driving systems from equilibrium shares many similarities from one method to the next. However, controlled experimentation on many of these systems is very difficult. Of great benefit to this effort would be placing an on-line accelerator at a synchrotron beam line to provide for in situ kinetic investigations. Such studies are needed because the number of control parameters and kinetic pathways is far too large to attempt examining specimens by ex situ methods.

It is worth mentioning that similar processing schemes occur and perform very important functions in biological systems. Self-assembly is one of the tools needed to make ordered nanostructures. Only the dynamics underlying the self-assembly process in this context are now well understood [14]. A material system embedding enormous complexity dynamically transits a variety of states, squeezing out free energy along the way, to arrive at a functional (and hopefully desirable) configuration. The spontaneous organization of a vesicle and the folding of a protein are notable examples of this phenomenon. Self-assembly, however, is not a completely enabling and sufficient tool. Biological systems make extensive use of both selfassembly and dissipative processes to make important structures or effect adaptive changes [15]. The double helix assembles, but complex processes involving substantial energy inputs from metabolism are needed to construct (or read, transcribe, etc.) the chromosome [16]. This central ability of the living system is far more complex than the driven systems described above; our science can neither imitate it broadly nor understand it in terms of detailed theory at this time. For example, how should one think about a system of objects not at equilibrium which arise via dynamically transforming interactions - a characteristic one expects for a mesoscale object obtained via assembly and dissipative processing? A substantial, and potentially high impact, materials research challenge suggested here is to devise ways for developing both complex structure and functions that mimic this powerful ability of the biological world [17].

\section{Phase Transformations}

Many processing schemes involve transformations between different phases of a material. Although thermodynamics ultimately determines the equilibrium phase for a given set of conditions, the answers as to whether and how this phase is produced from the metastable precursor phase depend on kinetics. Since most phase transitions involving nanostructured materials occur under conditions far from equilibrium, the kinetic pathways available to these systems are numerous and not well understood. This problem is compounded by the large contributions to the free energy of nanoscale materials from interfaces, which substantially shift phase boundaries. It will be important for making progress in the areas of synthesis and processing, therefore, to develop our understanding of nanochemistry and the broader general issues of nucleation and growth. 
One example of how phase transformations are used in processing nanostructured materials has already been noted, namely the formation of metallic nanoparticles in silicate glasses for nonlinear optical devices. Here, metal ions are implanted into glass and subsequently heat treated to precipitate out the metallic nanoparticles [10]. The processing of bulk nanostructured materials from bulk metallic glasses provides another important example of using phase transformations in nanotechnology. This processing scheme is becoming more attractive as the ability to form bulk metallic glasses broadens to a wider range of systems. Soft magnetic materials comprised of nanocrystalline bcc $\mathrm{Fe}-\mathrm{M}-\mathrm{B}(\mathrm{M}=\mathrm{Zr}, \mathrm{Hf}, \mathrm{Nb})$, for example, have been processed from the bulk glass, and they have excellent (soft) magnetic properties [18]. These materials are now commercially available. Despite this potential for processing of nanocrystalline bulk alloys, a general scheme for crystallization of amorphous alloys has not been developed beyond this single area of soft magnets. This is largely due to the difficulty of producing bulk glasses. Further research is needed in developing a far more extensive list of bulk glasses that can be synthesized. This will require a more fundamental understanding of the kinetics of nucleation and growth of crystalline phases in glasses, and a deeper understanding of the nature of glassy systems themselves.

A different class of phase transformations important to nanoscience and nanotechnology involves aerosol methods for synthesizing nanoparticles, such as inert gas condensation, flame pyrolysis, etc. These particles can be employed for a variety of applications: sensors, catalysts, sintered bodies, nanotubes and the like. While much work has been performed on controlling size and structure of elemental nanoparticles [19], an enormous potential for fabricating tailored, more complex particles, including alloys, compounds, and organics, currently exists. Presently, no general understanding is available for predicting the nanochemistry involved and surprisingly few fundamental in situ studies of the growth environment have been performed. Studies using in situ processing diagnostics will be necessary, however, to develop processes for truly controlled nanostructure growth.

\section{Time-Resolved, In Situ Diagnostics of Nanoparticle Formation}

A variety of time-resolved spectroscopic tools are currently being developed to understand gasphase nanomaterial synthesis and function. These include photoluminescence from nanoparticles; optical absorption spectroscopy and laser-induced fluorescence of ground-state molecules and precursors; Rayleigh scattering for nanoparticle sizing; plasma temperature measurements; and many more [20,21]. These techniques should be implemented to determine the precursor species responsible for nanomaterial growth, the vapor and heat transport processes involved in their growth, plasma density and temperature, nucleation temperatures and times, and nanomaterial growth rates.

Another example of a non-invasive, in-situ diagnostic of the quality, quantity and properties of nanoparticles is the use of small angle neutron scattering (SANS) to study the characteristics of nanodroplet aerosols formed by expanding a condensable vapor in a supersonic nozzle. The importance of aerosol SANS stems from its potential to probe directly the microscopic structure of nanometer-sized droplets [22]. Particles in this size range are the first condensed-phase fragments to appear during aerosol formation, and aerosol SANS affords us the first detailed, insitu look at them. Aerosol SANS measurements also allow the direct measurement of the particle size distribution of the aerosol and the speed of the particles in the expanding flow. All 
of these data were previously unavailable or had to be inferred from gas-dynamic flow measurements using unrealistic assumptions and simplified models. This information now provides an opportunity to perform the most stringent testing to date of theories of nucleation and droplet growth under the technologically important conditions of very high supersaturation and cooling rate found in turbine expansions, jet engine and rocket exhausts, and nozzle-based particle production techniques.

\section{Directed Synthesis of Nanoparticles, Nanotubes and Nanostructured Materials}

We note there is vast potential for developing directed synthetic chemistries that lead to very precisely defined nanoparticles appropriate for specific applications in technology. It has been shown, for example, that carbon-supported $\mathrm{Pt}-\mathrm{Ru}$ alloy nanoparticles can be prepared from molecular cluster precursors in a form appropriate for use as an electrocatalyst in Direct Oxidation Fuel Cells (DOFC). Perhaps most intriguing, the nanoscale alloy clusters made in this way show essentially no distribution in either the cluster size or composition, a feature which powerfully enables their detailed structural characterization by STEM and XAFS methods [23].

Nanostructured materials also promise greatly improved structural properties in comparison with conventional metal alloys. For example, small-diameter bundles of single-walled carbon nanotubes (SWNT) are predicted and observed [24,25] to have the largest strength-to-weight ratio of any known material with an elastic modulus $\sim 1 \mathrm{TPa}$, which is approximately one hundred times that of steel but with only one-sixth its weight. Such materials offer almost unimaginable economic benefits and product opportunities, if they can be cross-linked to overcome a low shear modulus [24] and synthesized by an economically competitive process that is compatible with other manufacturing technologies. It is clear this will require the development of new approaches for high-volume directed deposition of nanostructured materials. SWNT, for example, are not useful for structural applications unless they can be grown directly into strong composite structures 1000 times faster than current CVD methods. In particular, the carbon nanotube synthesis techniques under development for molecular electronics (see section 6) are not economically feasible for high-volume structural applications of SWNT composites. Consequently, theory, computer modeling and experimental diagnostics of transport and growth phenomena are needed in order to directly fabricate SWNT and other nanostructured construction materials at near-theoretical growth rates. 


\section{References}

1. J. R. Weertman, D. Farkas, K. Hemker, H. Kung, M. Mayo, R. Mitra, and H. Van Swygenhoven, MRS Bulletin, February 1999, pp. 44-50.

2. F. M. Ross, J. Tersoff, and R. M. Tromp, "Coarsening of self-assembled Ge Quantum Dots on Si(001)," Phys. Review Lett. 80, 984 (1998).

3. T. I. Kamins and S. Williams, "Lithographic positioning of self-assembled Ge islands on Si (001)," Appl. Phys. Lett. 71, 1201 (1997).

4. J. F. Wendelken, W. W. Pai, Z. Zhang, Bulletin of the American Physical Society Vol. 44, "Nanocluster Growth Under the Influence of Coulombic Forces," p.706 (1999).

5. L. Huang, S. J. Chey, J. H. Weaver, "Buffer-Layer-Assisted Growth of Nanocrystals: AgXe-Si (111)," Phys. Rev. Lett. Vol. 80, pp. 4095-4098 (1998).

6. P. Bellon and R. S. Averback, "Nonequilibrium Roughening and faceting of interfaces in Driven Alloys," Phys. Rev. Lett. 74, 1819 (1995).

7. G. Martin and P. Bellon, "Driven Alloys," Sol. State Physics 50189 (1997).

8. See e.g., C. C. Koch, in Materials Science and Technology, Vol. 15, ed. R. W. Cahn, P. Haasen, and E. J. Kramer, V. C. H Weinheim, p. 193 (1991).

9. R. Skomski and J. M. D. Coey, "Giant energy product in nanostructured two-phase magnets," Phys. Rev. B 48, 15812 (1993).

10. R. H. Magruder III, Y. Li, R. F. Haglund Jr, C. W. White, L. Yang, R. Dorsinville, R. R. Alfano "Optical properties of gold nanocluster composites formed by deep ion implantation in silica," Appl. Phys. Lett. 62, 1730 (1993).

11. D. M. Kelly, I. M. Schuller, V. Korenivski, K. V. Rao, K. K. Larsen , J. Bottinger, E. M. Gyorgy and R. B. Van Dover, "Increase in giant magnetoresistance by ion irradiation," Phys. Rev. B 50, 3481 (1994).

12. F. Garrido, J. C. Dran, L. Thome, C. Meneghini, F. Gonella, and A. Quaranta, "Highenergy ion-beam mixing: a new route to form metallic nanoclusters in a dielectric matrix," Nucl. Instr. Meths. B115, 561 (1996).

13. E. Chason, T. M. Mayer, B. K. Kellerman, D. T. McIlroy and A. J. Howard, "Roughening instability and evolution of the $\mathrm{Ge}(001)$ surface during ion sputtering," Phys. Rev. Lett. 72 3040 (1994).

14. C. L. Brooks III, M. Gruebele, J. N. Onuchic, P. G. Wolynes, "Chemical physics of protein folding," Proc. Natl. Acad. Sci. U. S. A., 95, 11037 (1998); J. N. Onuchic, Z. LutheySchulten, P. G. Wolynes, "Theory of protein folding: the energy landscape perspective," Annu. Rev. Phys. Chem. 48, 545 (1997).

15. H. V. Westerhoff, P. R. Jensen, J. L. Snoep, B. N. Kholodenko, "Thermodynamics of complexity," Thermochim. Acta. 309, 111 (1998); G. M. Whitesides, R. F. Ismagilov, "Complexity in chemistry," Science 284, 89 (1999).

16. B. Alberts, D. Bray, J. Lewis, M. Raff, K. Roberts, J. Watson Eds., "Molecular Biology of the Cell-3 $3^{\text {rd }}$ Ed.," (Garland Publishing, New York, NY), p 385-479.

17. I. S. Choi, N. Bowden, G. M. Whitesides, "Shape-Selective Recognition and SelfAssembly of mm-Scale Components." J. Am. Chem. Soc. 121, 1754 (1999). J. Tien, T. L. Breen, G. M. Whitesides, "Crystallization of Millimeter-Scale Objects with Use of Capillary Forces,” J. Am. Chem. Soc. 120, 12670 (1998).

18. Y. Yoshizawa, S. Oguma, and Y. Yamauchi, "New Fe-based soft magnetic alloys composed of ultrafine grain structure," J. Appl. Phys. 64, 6044 (1988); G. Herzer, "Grain structure and magnetism of nanocrystalline ferromagnets," IEEE Trans. Magn. 25, 3327 (1989). 
19. R. C. Flagan, M. M. Lunden, "Particle structure control in nanoparticle synthesis from the vapor phase," Mats Sci. \& Eng. A204, 113 (1995).

20. D. B. Geohegan, A. A. Puretzky, and D. J. Rader, "Gas-phase nanoparticle formation and transport during pulsed laser deposition of $\mathrm{YBa}_{2} \mathrm{Cu}_{3} 30_{7}-\mathrm{d}$," Appl. Phys. Lett. 74, 3788 (1999).

21. D. B. Geohegan, "Diagnostics and Characteristics of Pulsed Laser Deposition Laser Plasmas," p. 115 in Pulsed Laser Deposition of Thin Films (D. B. Chrisey and G. K. Hubler, eds.), John Wiley and Sons, New York, 1994.

22. B. E. Wyslouzil et al., "Small angle neutron scattering from nanodroplet aerosols," Phys. Rev. Lett. 79, 431 (1997); B. E. Wyslouzil and G. Wilemski, "Binary nucleation kinetics .3. Transient behavior and time lags." J. Chem. Phys. 105, 1090 (1996).

23. "Core Shell Inversion during Nucleation and Growth of Bimetallic Pt/Ru Nanoparticles," J. Am. Chem. Soc. 120, 8079 (1998). M. S. Nashner, A. Frenkel. D. L. Adler, D. M. Sommerville, J. R. Shapley, R. G. Nuzzo, "Structural Characterization of Carbon Supported $\mathrm{Ru}-\mathrm{Pt}$ Nanoparticles derived from the Molecular Precursor $\mathrm{PtRu}_{5} \mathrm{C}(\mathrm{CO})_{16}$," J. Am. Chem. Soc. 119, 7760 (1997).

24. J.-P. Salvetat, G. A. D. Briggs, J.-M. Bonard, R. R. Bacsa, A. J. Kulik, T. Stöckli, N. A. Burnham and L. Forro, Phys. Rev. Lett. 82, 944 (1999).

25. P. Poncharal, Z. L. Wang, D. Ugarte and W. A. de Heer, Science 283, 1513 (1999). 


\section{NANOSCALE PRECURSORS AND ASSEMBLY FOR MACROSTRUCTURES AND DEVICES}

\section{A. Introduction}

Nanocrystals and nanostructures will be the building blocks for future materials that will exhibit enhanced or entirely new combinations of properties for use in energy conversion and related technologies. However, there are two quite differently motivated categories for the assembly of macroscopic structures and devices from nanoscale materials. In the first category, the nanoscale materials are used to form a precursor state of the macroscopic system or device, and upon formation of the final state the nanoscale nature of the materials is severely altered or destroyed. In this approach the unique properties of the nanoscale materials are used to make the final synthesis of the macroscopic material easier, better, and/or more efficient. In the second category, the macroscopic system is composed of functional nanoscopic materials whose integrity is preserved. In this case, the properties of the ultimate macroscopic material, device, or system are derived from those of the nanoscale components, perhaps modified by how they couple when assembled into a macrostructure. These two categories are discussed further below.

\section{B. Nanoscale Precursors to Films for Electronic and Photonic Devices}

\section{Current Status}

Semiconductor nanocrystals 1 to $20 \mathrm{~nm}$ in diameter, synthesized via colloidal chemistry, have been used as precursor materials to produce thin films of electronic materials including photovoltaic cells [1-3]. The approach is to first spray a colloidal solution of semiconductor quantum dots onto a heated substrate to form a nanostructured semiconductor film. The film is then heat-treated at high temperature to melt the quantum dots and form macroscopic semiconductor crystallites which can be used in photovoltaic or other electronic devices. The advantage of this approach is that the melting points of semiconductor nanocrystals are markedly depressed compared to the bulk materials and the final semiconductor film can therefore be formed at much lower temperature. In addition, the electronic and optical properties of polycrystalline semiconductor films formed this way are often superior to films made by conventional deposition techniques. This approach has been successfully applied to photovoltaic films of $\mathrm{CdTe}[1,2]$ and $\mathrm{Cu}(\mathrm{In}, \mathrm{Ga}) \mathrm{Se}_{2}$ (CIGS) [3] and used to form contacts to $\mathrm{Si}$ [4] and CdTe $[4,5]$.

Another application using the same principle is the use of semiconductor nanocrystal precursors to make photonic bandgap materials [6]. In this application, colloidal semiconductor quantum dots 3-4 nm in diameter are deposited in the interstitial spaces of synthetic opals consisting of ordered arrays of silica spheres $250 \mathrm{~nm}$ in diameter. The quantum dots self-assemble into a quantum dot solid inside the silica opal. The silica is then dissolved away leaving a macroporous solid consisting of densely packed semiconductor nanocrystals. The nanocrystals are then sintered to form a macroporous bulk semiconductor that has a periodic modulation of the refractive index on the length scale of $250 \mathrm{~nm}$, which makes it an ideal candidate for photonicbandgap applications. Nanostructured films and materials are potentially very useful in other 
applications. Metal oxide nanoparticles have been used to fabricate nanostructured cathodes for lithium battery applications using the same spray deposition strategy outlined above. Metal and metal oxide nanoparticle catalysts are also being examined.

\section{Scientific Issues}

The main challenge in using nanocrystal precursors to fabricate macroscopic films is in developing the processing technique that converts the precursor film into a high-quality largegrained film. In the case of CdTe, treatment of the precursor film with $\mathrm{CdCl}_{2}$ prior to annealing facilitates the formation of large-grained, dense material. An analogous approach with ternary or quaternary semiconductor materials, such as CIGS, has not been as successful. New processing strategies must be found for these materials.

The synthesis of photonic bandgap materials also requires much better understanding and control of the mechanism for creating a bulk semiconductor material from quantum dot precursors upon melting. For photonic bandgap applications the conversion process must produce periodic semiconductor structures with the high refractive index of the bulk material.

\section{Future Directions}

Nanoparticle precursors are likely to be used to fabricate all of the components in future photovoltaic device structures. They will be especially useful for depositing films on top of thermally sensitive underlayers and on new substrates such as plastics. Deposition of semiconductor films will be extended to include materials other than CdTe and CIGS and nanocrystal precursors will be developed for depositing transparent conducting oxides, metals and other electrical contact materials. Metal nanoparticle inks will be developed that will allow deposition of metal lines using inkjet or other printing techniques. Spray deposition of nanoparticle precursors can also have an impact in other areas, including the application of inorganic coatings. The ability of quantum dot precursors to make new photonic bandgap materials with excellent properties will lead to new and/or better optical elements that may enable several opto-electronic applications, including non-linear optical devices, information storage and transmission, and optical computing systems.

\section{Macroscopic Structures Comprised of Functional Nanoscopic Materials}

\section{C.1 Nanoscale Computing, Interconnection, and Input/Output (I/O)}

\section{Current Status}

The electronic industry has, for the last 35 years, increased the logic density of transistors on a chip with a doubling time of approximately 18 months (Moore's Law). This has been possible because of the continuous shrinking of the minimum feature size that can be generated with lithography tools. The trend has been to use shorter-wavelength photons to generate the necessary images. Current standard technology employs UV (pulsed excimer laser) light with a wavelength of $248 \mathrm{~nm}$ to generate 0.25 -micrometer features. The state-of the-art achieves features of about 0.18 micrometers by using a shorter (ArF, $193 \mathrm{~nm}$ ) laser wavelength. However, the absence of optical materials to transmit and focus still shorter-wavelength photons has resulted in electron beam and X-ray lithography now being considered for even smaller 
feature size. The overwhelming growth in the complexity and cost of chip manufacturing provides clear evidence that economic limits to continually shrinking feature size also are rapidly being approached. However, additional miniaturization is driven inexorably by the need for higher speed, reduced power consumption, reduced size, and increased capacity and logic density in future-generation computer systems. Thus, three promising alternatives, quantum dots, molecular electronics, and ultra-small Josephson junctions are being considered as alternatives to the conventional silicon integrated circuit (IC) paradigm.

Metal, semiconductor or superconductor islands, a few nanometers diameter, surrounded by an insulating layer can operate as single-electron devices [7]. This property can be used for a variety of applications such as computation, detection, and remote sensing. In addition, coupling single devices into arrays opens new technological horizons, enabling collective effects to be used for coherent high-intensity radiation, for communications, and for computation.

Quantum dots (QD) can be configured to operate as transistors or associative memory elements through appropriate fabrication processes. Unlike conventional transistors that operate by moving ensembles of electrons, QD devices utilize quantum mechanical phenomena that emerge only as dimensions are reduced to the nanometer scale [8]. In particular, electron movement becomes a discrete phenomenon involving one electron at a time. It is suggested that quantum dots, fabricated in two-dimensional arrays, will lend themselves to nanoelectronic operation of advanced computer architectures such as neuromorphic networks. Josephson junctions [9] are natural voltage-to frequency transducers. These devices have a broad range of applications, the most prominent one being SQUIDs (superconducting quantum interference devices [10]), which are used to measure extremely small magnetic fields. In addition to today's well-established biomedical and research applications, SQUIDs are making inroads in nondestructive evaluation, explosive detection, and geophysics [11]. Small and ultra-small Josephson junctions are promising for nanodevice applications because (a) these are intrinsically quantum devices (even on the macroscopic length [12]); (b) their very high operating frequencies are capable of probing other nano-devices; and (c) they are able to emit and detect coherent radiation in the terahertz range.

Low-capacitance Josephson junction arrays, in the parameter range where single charges can be controlled, have the potential to provide a physical realization of quantum bits and, therefore, can be considered as a component for quantum computation. The hope is that they can provide, for instance, a basis for a controlled quantum logic gate [13], which has a natural implementation in terms of Cooper pair transport. Future research is required to establish connections between arrays of small Josephson junctions and quantum computation and quantum state engineering. In addition to the manipulation of qubits, the resulting quantum state has to be read out. The possibility of coupling Josephson junctions to other electronic devices (such as, for example, single-electron transistor and quantum dots) needs to be better understood [14]. 
Molecular Electronics. The vision of building dense, small, and fast computing elements composed of more than $10^{15}$ operational molecular-or-atomic scale components is predicated on having the ability to self-assemble and interconnect these nanodevices with an acceptable yield. In fact, even mainstream silicon IC technology may be limited by the interconnect problem before being limited by lithographic constraints [15], and the interconnect problem is even more severe for very dense molecular-scale devices. It is difficult to envision supercomputers the size of a grain of sand [16] unless breakthroughs are made in the interconnect and I/O areas.

The underlying concept of nanofabrication emerged as one of the important uses of scanning probe microscopes (SPM) [17] which, in a relatively short time have become powerful tools both to image and to manipulate matter at the atomic scale. Localized chemical changes are in most cases an essential component of SPM nanofabrication procedures. The change results from a molecular species being modified at localized sites by a pulsed electrical potential transmitted by the SPM tip. Representative examples include the production of metal-oxide-metal junctions by anodic oxidation of a previously evaporated titanium film, or the fabrication of nanowires of a TaIr alloy after etching an over-layer of amorphous silicon patterned in an oxidation step. Conversely, it also has been possible to create features on silicon and gallium arsenide with the tip of the microscope submerged in a dilute acid solution, which etched immediately the oxide generated by the tip potential. Alternatives to nanofabrication by SPM are the deposition of metal dot arrays through self-assembly, or the evaporation and selective etching of metal films. These metal dot arrays lend themselves to operation as resonant tunneling diodes [18].

The two principal materials and approaches being considered for molecular wiring at this time are organic molecular wires [19] and carbon nanotubes (CNTs) [20]. Molecular wires have the advantage of high volume ("beaker-scale") fabrication and can be easily chemically modified to allow chemical self-assembly [21]. However, recent research has shown that the low power dissipation, remarkable strength and very small $(\sim \mathrm{nm})$ diameter of CNTs may be uniquely suited to both molecular wiring and I/O. For molecular wiring CNTs can be cut, separated by length, chemically functionalized, and bonded to gold substrates or nanoparticles [22]. In addition, ordered arrays of CNTs have been grown on substrates [23]. Arrays of CNT field emitters, for example, may provide a vital link in molecular-scale I/O.

\section{Scientific Issues and Challenges}

The central scientific issue and problem can be broken naturally into two parts:

(1) the interconnection of molecular devices to each other (molecular wiring); and

(2) the connection of molecular devices to bulk substrates and structures that allow the introduction of signals $(\mathrm{I} / \mathrm{O})$.

Significant challenges for both of these areas include:

(1) the need for directed self-assembly of interconnects into coherent circuits with acceptable yield;

(2) understanding and controlling charge transport across dissipative device/wire and wire/substrate interfaces;

(3) understanding and controlling the electrical properties of molecular wires at different length scales ( $\mathrm{nm}$ to $\mu \mathrm{m}$ );

(4) characterizing the electrical properties of nanoscale devices in order to extract models for computer simulation of complex molecular circuits; and 
(5) making sufficiently rugged interconnect devices that can operate outside of controlled environments.

\section{Future Directions and Promise}

Quantum Dots. Research is needed to further our understanding of nanosystem fabrication, and to develop mathematical models and algorithms to study the electronic properties and computational capabilities of single-electron device arrays. From an application perspective, early consideration should be given to neuromorphic architectures for associative memory. The following research program elements are envisioned for quantum dots:

(a) Generation of metal dots. Note here that the considerable difference in conductivity between metal and surrounding oxide may provide a new means to image features.

(b) Minimum feature size achievable. Optimization processes must be developed to establish the smallest feature that can be fabricated. Variables to be studied may include the effect of film thickness, substrate thermal characteristics, film adherence and carbon doping and annealing treatments.

(c) Device architecture. Physical effects in nanoscale structures play a major role in achieving logic functionality not attainable with current technology. To optimize future nanoelectronic architectures, quantum transport models and simulations must be developed that include these physics effects.

(d) Neuromorphic computational architectures and algorithms. The dynamics of nanoelectronic networks should be studied with the objective of deriving a common formalism to describe these networks and configure neuromorphic systems. The practical goal would be to discover a mechanism by which neural networks of increasing dynamical complexity could be embedded in an assembly of nanoscale devices. Computational models for the study of effects of random dot dispersion should also be developed and evaluated.

Josephson junctions. Recently it was reported [24] that a two-dimensional array of $\mathrm{Nb} / \mathrm{Al} / \mathrm{AlO}_{\mathrm{X}} / \mathrm{Nb}$ Josephson junctions (junction area $16 \mu \mathrm{m}^{2}$ ) arranged in square geometry (spacing between junctions $13 \mu \mathrm{m}$ ) emits coherently in a novel synchronized state. Above the threshold, the power scales as $\mathrm{N}^{2}$ which suggest that the array is in an in-phase synchronized state consistent with the stimulated emission causing coherence and suggesting population inversion. Theoretical background predicting analogies between junctions and coherent atomic systems was developed in the 1970s, but the experimental confirmation came only very recently. This result opens a new way of looking at an array of Josephson junctions as a laser with the frequency range of hundreds of gigahertz to terahertz. The next step in this research would be to establish whether arrays of much smaller, nano-size junctions, could emit coherent radiation, and estimate the resulting total power. In particular, the question arises whether Josephson arrays can be used as emitting devices for quantum teleportation. Both numerical simulations and theoretical and experimental research are desired to advance the research in this field. Because these arrays are intrinsically nonlinear and possess quantum (and perhaps, classical) dynamics, a deeper understanding in basic quantum and classical nonlinear dynamics and chaos is required [25-29].

Molecular Electronics. Individual covalently bonded molecules provide a unique ultra-dense, low-power framework for purely nanoelectronic circuitry, including switching devices and 
wires. A primary advantage of molecular electronics is that molecules are natural nanometerscale structures that can be made absolutely identical in vast (e.g., 10 $0^{23}$ ) quantities [30]. Research is needed to understand and harness the electronic properties of organic molecules, and to develop the molecular analogs of solid state nanoelectronic devices. An example of a potential benefit from achieving switching devices of $1-2 \mathrm{~nm}$ feature size and densities in excess of $10^{12}$ devices per $\mathrm{cm}^{2}$ would be a terabit memory on a chip. Factors determining controlled self-assembly, interconnectivity (wiring), and corresponding modeling based on first principles are high priority research efforts.

The challenge and importance of molecular interconnection and $\mathrm{I} / \mathrm{O}$, coupled with the recent advances in CNT fabrication and manipulation, constitute a major research opportunity. Teams of DOE researchers including electrical engineers, chemists, solid-state physicists and computer scientists could make substantial progress in intermolecular connectivity and macroscale-tonanoscale communications. Such breakthroughs are essential to full-scale practical implementation of nanoscale devices and will provide key enabling technology for the entire nanoscience and nanotechnology initiative.

\section{C.2 Macroscopic Structures Containing Ion Implanted Nanostructures}

\section{Current Status}

Ion implantation has been used to create nanocrystals and quantum dots that are encapsulated near the surface of a variety of host materials [31]. This work was motivated by the unique optical and electronic properties of nanocrystals which result from quantum confinement and the fact that ion implantation can be utilized to create high densities of an enormous variety of elemental and compound nanocrystals (metals, elemental semiconductors, compound semiconductors, and alloys) embedded in a wide range of host matrices that include $\mathrm{SiO}_{2}, \mathrm{Al}_{2} \mathrm{O}_{3}$, $\mathrm{Si}$, and $\mathrm{MgO}$. In some cases, it has been possible to control both the structure and orientation of the nanocrystals [32]. Ion implantation actually may be the simplest way to form many nanocrystals due to their sensitivity to high temperatures or oxidizing conditions. Three new areas of nanotechnology $R \& D$ are discussed below.

\section{Scientific Issues and Future Directions}

Ordered Arrays of Uniform-Size Nanoparticles Formed by Finely Focused Ion Beam (FFIB) Implantation. The nanoparticle size distribution is critically important for many applications but is difficult to control using large-area ion implantation. However, novel optical materials could be formed by using FFIB (down to $5 \mathrm{~nm}$ beam diameter) to "write" individual colloidal semiconductor particles of constant size arranged in a 2-dimensional lattice. The advantages of this approach would stem principally from the uniform size of the particles, the accurate spacing of the lattice, and the ability to control precisely both the size and the spacing. Such materials would have numerous applications as photonic crystals in lasers, mirrors, and waveguides and, because of the flexibility of the focused ion beam, could be tailored to suit individual applications. In this method, a FFIB will be used to implant an ordered array of spots. Subsequent annealing leads to nucleation and growth of nanoparticles with the composite relaxing toward a single particle size as suggested by kinetic Monte Carlo simulations similar to those carried out for large-area implants [33]. In the FFIB approach, particle size is controlled by the local dose, which should allow nanocrystal size distributions to be controlled within a few 
atoms, the narrowest ever produced for nanocrystal ensembles. The same techniques would be applied to form nanowires.

Dual-Beam Irradiations for Manipulation and Control of Nanostructured Material Formed by Implantation. Properties of nanoparticles depend critically upon their size, size dispersion, spatial distribution, and crystallographic orientation. Consequently, it is imperative to develop methods to control these characteristics. It is proposed that a secondary "energizer" beam be used simultaneously with the ion source beam during formation of nanoparticles by ion implantation. Supplemental energy deposited by an energizer beam generates point and extended defects as well as electronic and vibrational excitations that substantially change the kinetics of nanoparticle nucleation and growth, thereby altering their size and orientation [34]. It is anticipated that use of an appropriate energizer beam during implantation will yield uniformly sized particles that are crystallographically oriented within the host. This will require that the energizer beam be matched to the primary beam to ensure that it "activates" the desired property. This technique could be coupled with the "Smart Cut" method [35] for thin-film transfer by using hydrogen ions as the energizer. In this way, the energizer would not only manipulate the nanoparticle distribution but allow the fabricated devices to be separated and readied for assembly in sensors or other devices.

Integration of "Ion Slicing" and 3D Wafer Bonding for Assembly of Nanoscale Devices. Many components of nanoscale devices are likely to be grown either attached to or embedded near the surface of solid substrates. In order to fabricate three-dimensional (3D) nanoscale devices from such components, it will be necessary to separate them from the substrates and attach them to the rest of the device in the desired configuration, particularly in cases where means for self-assembly have not been devised. Integration of methods such as "ion slicing" for separation and wafer bonding between 3D nanostructures provides a potential path to assembly of nanoscale devices. The term "ion slicing" refers generically to a class of ion-related processes used to separate or cut thin films from bulk wafers, and includes the "Smart Cut" process [35] used in silicon microelectronics. Contact bonding of the thin film to another substrate prior to separation will result in the transfer of the film to this substrate. While such processes have been demonstrated for a number of materials, including diamond, silicon, and various ferroelectric oxides [36], their applicability to a wider range of materials needs to be explored. General issues of dimensional control and uniformity also must be resolved to advance this technology to the nanoscale. Critical issues include bonding materials that have 3D or surface relief structure, and the alignment or registry of the nanostructures prior to bonding. This will require integration of standard patterning and lithographic techniques with the new transfer methods. The result will be to open a path to efficient fabrication of nano-devices by utilizing large-area substrates to facilitate their handling and to enable parallel simultaneous fabrication of large numbers of nanoscale devices.

\section{Nanomechanics and Nano-to-Micro Assembly}

\section{Current Status}

The future of nanoscale devices depends upon the scientific community's capacity to physically manipulate nano-sized parts and to integrate larger micro-to-mini scaled devices [37]. 
However, the relative importance of fundamental physical forces changes as parts are reduced in size.

Figure 6.1 shows the variation of forces with respect to scale. When handling miniature parts with features on the order of a few millimeters, classical mechanical phenomenon such as mass and friction dominate the mechanics of manipulation. However, when part sizes are reduced below the millimeter threshold, surface effects such as adhesion due to surface condensation and the electrostatic potential between parts dominate over the classical inertial effects

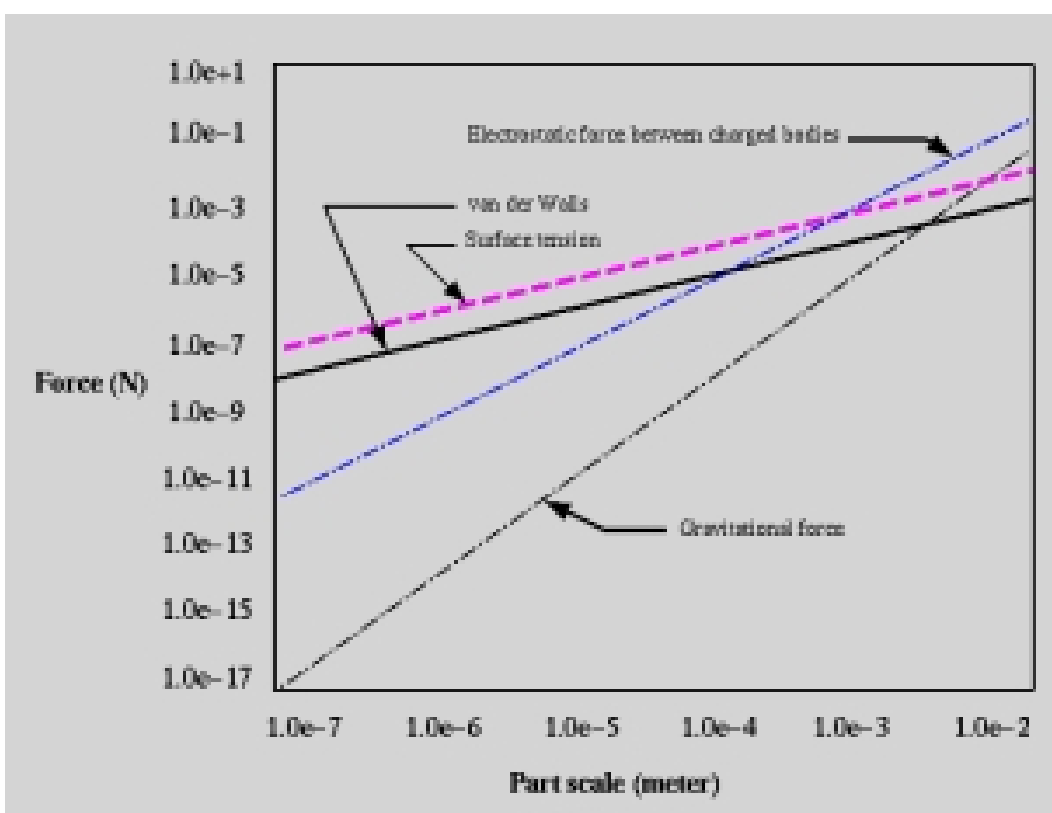

Figure 6.1: Force variation with scale present in large-scale moving structures. Research currently focuses on the assembly of millimeter-to-micron-sized parts [38]. Figure 6.2 shows a series of gears with features below a millimeter and with clearance fits between the parts of less than a micron. At this level surface forces become relevant but it is still possible to use many classical techniques for successful manipulation and assembly. However, we expect paradigm shifts in the methods of assembly as the parts become even smaller [39]. Examples include new methods of "visualizing" the parts as well as for manipulation and handling [40].

\section{Scientific Issues and Future Directions}

Nanomechanics may be as important as nanoelectronics. Cantilever beams can measure stress, heat, mass, and NMR. A linear array of cantilevers, when treated with selectively adsorbing coatings, can distinguish among various chemical species, and can act as an artificial nose. Microcantilevers can function as RF switches. A long-term goal for information storage is to have the capacity of $1000 \mathrm{CDs}$ in a device the size of a wrist watch. Large arrays of microcantilevers can be envisioned as high-density storage devices (40 Gbits/cm) with presentday technology. With further research, it may be possible to get to $400 \mathrm{Gbits} / \mathrm{cm}$ using carbon fullerenes as the cantilever elements in large arrays [41]. 


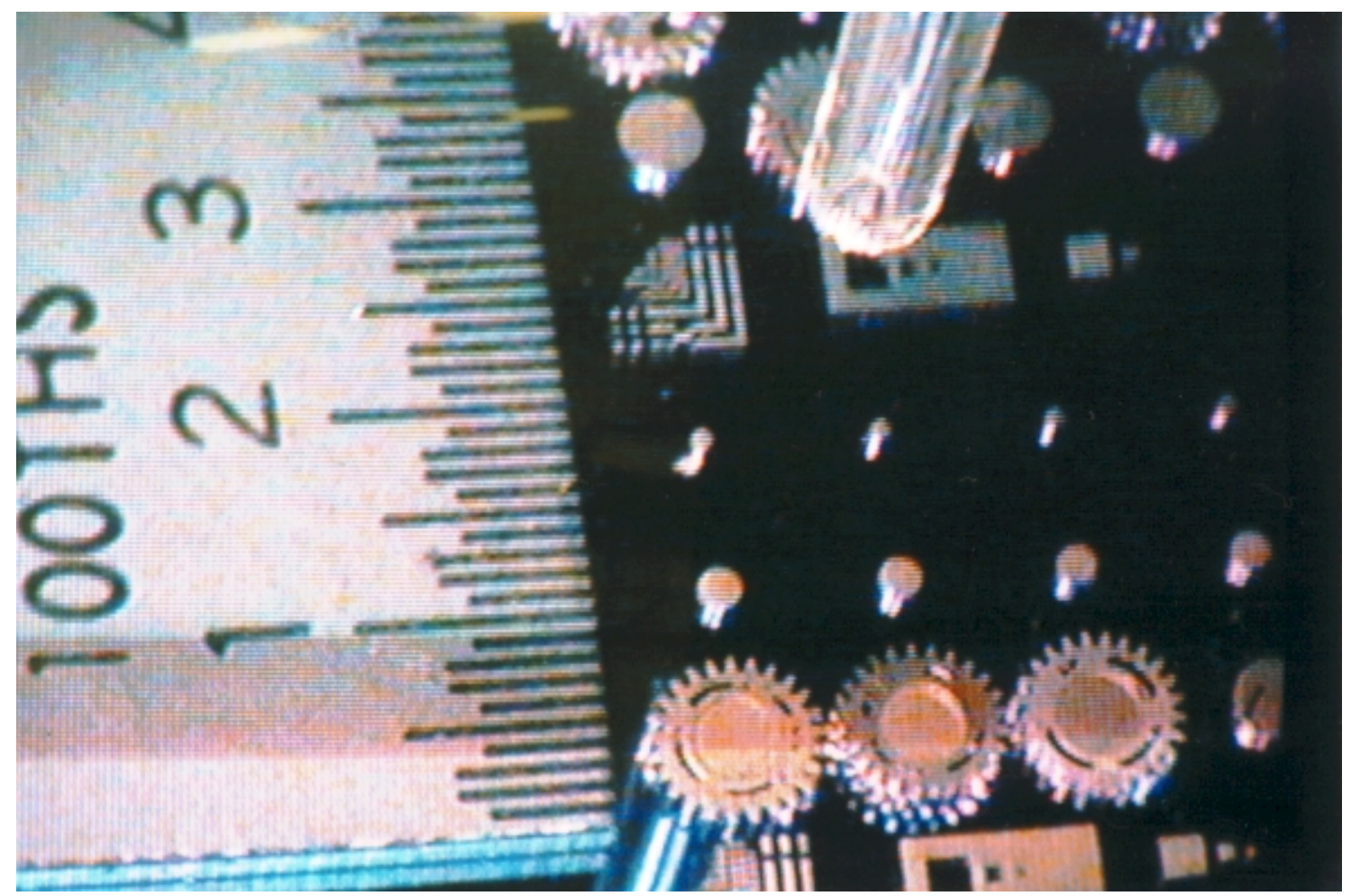

Figure 6.2: Assembly of micro gears

\section{References}

1. M. Pehnt, D. L. Schulz, C. J. Curtis, K. M. Jones, and D. S. Ginley, "Nanoparticle precursor route to low-temperature spray deposition of CdTe thin films," Appl. Phys. Lett. 67, 2176-8 (1995).

2. D. L. Schulz, M. Pehnt, D. H. Rose, E. Urgiles, A. F. Cahill, D. W. Niles, K. M. Jones, R. J. Ellingson, C. J. Curtis, and D. S. Ginley, "CdTe Thin Films from Nanoparticle Precursors by Spray Deposition," Chem. Mater. 9, 889-900 (1997).

3. D. L. Schulz, C. J. Curtis, R. A. Flitton, H. Wiesner, J. Keane, R. J. Matson, K. M. Jones, P. A. Parilla, R. Noufi, D. S. Ginley, "Cu-In-Ga-Se Nanoparticle Colloids as Spray Deposition Precursors for $\mathrm{Cu}(\mathrm{In}, \mathrm{Ga}) \mathrm{Se} 2$ Solar Cell Materials," J. Electron. Mater. 27, 433 (1998).

4. D. L. Schulz, R. Ribelin, C. J. Curtis, and D. S. Ginley, "Particulate Contacts to Si and CdTe: Al, Ag, Hg-Cu-Te, and Sb-Te," 1998 National Center for Photovoltaics Annual Review AIP Conference Proceedings, (in press).

5. D. L. Schulz, R. Ribelin, C. J. Curtis, D. E. King, and D. S. Ginley, "Nanoparticle-Based Contacts to CdTe," Materials Research Society Symposia Proceedings, (in press).

6. D. J. Norris N. Yao, and Y. A. Vlasov, APS Abstracts, Atlanta Meeting 44, 1181 (1999); Adv. Mat. 11, 185 (1999).

7. D. Bimberg, M. Grundman, N. N. Ledenstov, "Quantum Dots-a Review," Phys. Rpts. 243, 73 (1993); see also "Quantum Dot Heterostructures," John Wiley \& Sons, (1998).

8. S. Gandopadhyay, B. R. Nag, "Energy Levels in Three-Dimensional QuantumConfinement Structures," Nanotechnology 8, 24 (1997).

9. B. D. Josephson, "Possible New Effects in Superconductive Tunneling," Phys. Lett. 1, 251 (1962). 
10. C. D. Tesche, "Macroscopic Quantum Coherence: An Experimental Strategy," in SQUID '85, H. D. Halbohm and H. Lubbig, "Superconducting Quantum Devices and their Applications," Eds., pp. 355-359, Berlin: deGruyter, 1985.

11. J. Oulette, "SQUID Sensors Penetrate New Markets," The Industrial Physicist, pp. 20-24, June 1998.

12. "Quantum Phenomena in Circuits at Low Temperature," Prog. Low Temp. Phys. 8, 219 (1992).

13. A. Shnirman and G. Schön, "Quantum Measurements Performed with a Single-Electron Transistor," Phys. Rev. B 57, 15400 (1998); see also A. Shnirman, G. Schön, and Z. Hermon, "Quantum Manipulations of Small Josephson Junctions," Phys. Rev. Lett. 79, 2371 (1997).

14. S. Adourian, S. Yang, R. M. Westervelt, K. L. Campman, and A. C. Gossard, "Josephson Junction Oscillators as Probes of Electronic Nanostructures," Journal of Applied Physics 84, 5808 (1998).

15. The Semiconductor Industry Association (SIA) 1997 National Technology Roadmap.

16. Introduction to the Interagency Working Group on Nano Science, Engineering and Technology Report, "Vision for Nanotechnology R\&D in the Next Decade."

17. L. T. Hansen, A. Kuhle, A. H. Sorensen, J. Bohr, P. E. Lindelhof, "A Technique for Positioning Nanoparticles Using an Atomic Force Microscope," Nanotechnology 9, 337 (1998).

18. R. H. Blick et al., "Resonant Experiments in Strongly Coupled Quantum Dots," Phys. Rev. Lett. 80, 4032 (1998); see also T. H. Oesterkamp et al., "Resonant Spectroscopy on a Quantum Dot Molecule," LANL cond-mat . 98042 (1998).

19. L. A. Bumm, et al., "Are Single Molecular Wires Conducting?" Science 271, 1705-1707 (1996).

20. S. Iijima, "Helical Microtubules of Graphitic Carbon," Nature 354: (6348) 56-58 (1991).

21. N. S. Hush, et al., "Optimization and Chemical Control of Porphyrin-Based Molecular Wires and Switches," in Molecular Electronics: Science and Technology, A. Aviram and M. Ratner, Editors, Annals of the New York Academy of Sciences, Vol. 852, 1998.

22. J. Liu, et al., "Fullerene Pipes" Science 280, 1253-1256 (1998).

23. Z. F. Ren, et al., "Synthesis of Large Arrays of Well-Aligned Carbon Nanotubes on Glass," Science 282, 1105-1107 (1998).

24. P. Barbara, A. B. Cawthorne, S. V. Shitov, and C. J. Lobb, "Stimulated Emission and Amplification in Josephson Junction Arrays," Phys. Rev. Lett. 82, 1963 (1999).

25. Y. Braiman, K. Weisenfeld, "Global Stabilization of a Josephson Junction Array," Phys. Rev. B 49, 1 (1994).

26. Y. Braiman, W. L. Ditto, K. Weisenfeld, M. L. Spano, "Disorder Enhanced Stabilization," Phys. Rev. Lett. A 206, 54 (1995).

27. A. S. Landsberg, Y. Braiman, K. Weisenfeld, "Disorder and Synchronization in a Josephson Junction Plaquette," Appl. Phys. Lett. 67, 1935 (1995).

28. A. S. Landsberg, Y. Braiman, K. Wiesenfeld, "Effect of Disorder on Synchronization in Prototype Two-Dimensional Josephson Arrays," Phys. Rev. B 52, 15458 (1995).

29. Y. Braiman, F. Family, H. G. E. Hentschel, "Discrete Voltage States in One-Dimensional Parallel Array of Josephson Junctions," Appl. Phys. Lett. 68, 3180 (1996).

30. W. Reinherth et al., "Molecular Scale Electronics: Synthesis and Testing," Nanotechnology 9, 246 (1998).

31. C. W. White et al., "Encapsulated Semiconductor Nanocrystals Formed in Insulators by Ion Beam Synthesis," Nucl. Instrum. Methods Phys. Res. B 141, 228 (1998). 
32. J. D. Budai et al., "Controlling the Size, Structure, and Orientation of Semiconductor Nanocrystals Using Metastable Phase Recrystallization," Nature 390, 384 (1997).

33. M. Strobel, S. Reiss, and K.-H. Heinig, "Evolution of Nanocluster Ensembles: Computer Simulation of Diffusion and Reaction Controlled Ostwald Ripening," Nucl. Instrum. Methods Phys. Res. B 120, 216 (1997).

34. G. Martin, "Contribution of Dissipative Processes to Radiation-Induced Solid-Solution Instability," Phys. Rev. B 21, 2122 (1980).

35. M. Bruel, "Application of Hydrogen Ion Beams to Silicon On Insulator Material Technology," Nucl. Instrum. Methods Phys. Res. B 108, 313 (1996).

36. Q.-Y. Tong and U. Gösele, Semiconductor Wafer Bonding; Science and Technology, John Wiley \& Sons, New York, Chap. 12.5. (1999).

37. K. Eric Drexler, John Wiley \& Sons, "Nanosystems," Molecular Machinery, Manufacturing \& Computation, (1992).

38. L. Love, "Design and Control of a Force Reflecting Micro-Assembly System," Fourth World Automation Conference, Maui, Hawaii (in press, June 2000).

39. R. C. Merkle, “Convergent Assembly," Nanotechnology 8, 23 (1987).

40. M. Hoummady, H. Fujita, "Micromachines for Nanoscale Science and Technology," Nanotechnology 10, (in press, March 1999).

41. O. G. Collins, H. Bando, A. Zettl, "Nanoscale Electronic Devices and Nanotubes," Nanotechnology 9, 153 (1998). 


\section{UNDERSTANDING AND MIMICKING BIOLOGICAL FUNCTIONS: "DRAWING INSPIRATION FROM NATURE"}

It is unlikely that anyone thinks of a whale while considering nanoscience or nanotechnology. Even bacteria inhabit the micron regime. But living organisms are simply collections of atoms, molecules, and structures that are very much a part of the nanometer world. Tenth-nanometer ions such as potassium and sodium generate our nerve impulses. Sugars, amino acids, hormones and DNA are about one nanometer in size. Membranes that separate one cell from another or one subcellular organelle from another are about five-fold bigger. Proteins can be tens of nanometers across. The organism can do what it does, and cannot do what it does not, because of the presence, absence, concentration, location and interaction of these "nano-structures".

Dimensions notwithstanding, the study of nanoscience and nanotechnology does not, at least at this point, involve the use of intact living systems, either alone, as building blocks of structures, or as devices. It does, however, look to biological molecules for tools and components, and to biology broadly, for inspiration about what can be done. The uses of biological molecules and biology fall into four categories:

- Biological molecules can be used in conjunction with other structures to perform just as they do in organisms.

- Biological molecules can be used in conjunction with other structures to perform in a novel manner, quite distinct from their natural function.

- Biology can provide examples of approaches and solutions to specific problems.

- Biology can provide inspiration by demonstrating what types of problems have been solved by Nature (however poorly we might understand that solution) and therefore what problems might well be solved in our laboratories (with a lot more creativity and hard work).

\section{A. Current Status}

Examples have already been reported of successful efforts in each of these four categories. But whatever has been achieved pales in the face of the far greater opportunities that remain for exploitation, especially in the latter categories.

Functional nanostructures can incorporate individual biological molecules. For example, biosensors can use natural sugars or proteins as target recognition groups [1]. Modified biological structures can be used to act in devices-for example the use of modified photosynthetic membranes to split water to hydrogen and oxygen [2].

Functional multi-component structures can be fabricated that use molecules in unusual ways. The filament protein actin, found for example in muscle, can be attached to the enzyme ATP synthase, which is involved in the production of most of the cell's ATP, its "energy currency". 
The entire structure can then be immobilized on a glass slide. The natural rotation of the ATP synthase, powered by ATP hydrolysis, can be used to whip the actin filaments around in a circle, much as one would swing a lariat. The turning is precise: $120^{\circ}$ for each ATP molecule hydrolyzed [3].

Biological approaches to problems guide us in designing non-biological systems. The concept of combinatorial chemistry - the simultaneous synthesis on a chip of huge numbers of variants of a given structure, followed by the rapid screening of those variants for the few with optimal properties - draws its inspiration from the mechanism of antibody production by the immune system. In higher organisms, the $10^{10}$ different antibodies present in the body are synthesized by, in effect, linking the individual sections of the molecule. Each individual section is selected from a library of variants of that section [4]. In combinatorial chemistry, the variants of the inorganic material to be made are created by using the vast number of possible amounts of its four, five, six or more constituent elements [5]. In both cases, the population produced is rapidly screened to allow identification and then mass production of the variant that best serves the desired function and then it is mass-produced.

\section{B. Key Questions}

Many specific tasks performed by living systems use nanometer-size structures in particularly intriguing ways - performing functions that we would very much like to emulate.

- Organisms sense their molecular surroundings, having developed exquisitely sensitive nanometer-size sensors on their outer surfaces. Using the principle of molecular recognition, only the specific desired target can bind to the surface-mounted "receptor" molecules. Upon binding, these receptors change shape in a manner that alerts other components of the system, inside the cell, to the presence of the target.

- Living systems use nanometer-size structures to act as highly selective pumps. The electron transport chain, which is central to the trapping of the energy content of nutrients, pumps protons from one side of the mitochondrial membrane to the other, against a gradient [6]. Neurons pump sodium ions out and potassium ions into the cell to ready it for the next impulse [7].

- $\quad$ Living systems use nanometer-size structures as switches. Within $200 \mathrm{msec}$ of the binding of a repellant molecule to a receptor, a phosphoryl group is transferred to a protein and the rotation of the bacterial "tail" is switched from counterclockwise to clockwise. This turns the organism around and allows it to swim away from the repellant [8].

- Living systems use nanometer-size structures to perform catalysis, with specificity, selectivity and rate enhancements that are rarely if ever achieved artificially. Enzymes can be selective enough to catalyze a reaction with only one particular molecule, even if it is present in a mix of many, ignoring even its mirror image. Enzymes can selectively catalyze only one of many chemically allowed reactions with that molecule, with rate enhancements up to $10^{16}$ [9].

More broadly, and with more general potential impact, living systems exhibit a number of characteristics that can serve as models for nanoscience study. In some cases, our understanding 
of this behavior simply alerts us to a concept we may not have considered. In all cases, these are extraordinarily sophisticated but also very desirable properties that we would wish to incorporate into structures and devices to be used in non-biological settings. The very fact that Nature has succeeded in developing systems with these characteristics (albeit over hundreds of millions of years of evolution) says that they can be achieved - they are not unattainable goals. On the other hand, it must be accepted that in some cases, these goals may not be achievable except by living systems.

- Living systems exert precise control of the atomic composition and architecture of large and complex functional structures. Protein complexes as large as 10,000 cubic nanometers can be rendered nonfunctional by the change of a single atom.

- $\quad$ Living systems sense their surroundings and report that information to control systems for the appropriate response.

- $\quad$ Living systems can sense damage and repair it to its original functional state.

- $\quad$ Living systems can switch among several states in response to short term and reversible changes in their environment.

- Living systems can evolve over time, adopting new forms in response to long term, permanent changes in their environment.

- $\quad$ Living systems can self-assemble thousands of individual parts in a precisely defined functional structure.

- $\quad$ Living systems can build complex structures hierarchically. Collagen, a fiber that rivals steel in strength, is built through successive aggregation of single amino acid strands into triple helices, triple helices into microfibrils, microfibrils into fibrils, fibrils into fibers.

- $\quad$ Living systems use templates, such as DNA, as blueprints for structures where there is no thermodynamically or kinetically available selection for that structure.

- $\quad$ Living systems can create a low entropy structure of importance by increasing the entropy of the entire system. Protein folding, creating a highly ordered arrangement of the constituent amino acids, is driven by the maximization of the entropy of the surrounding water.

- $\quad$ Living systems can reproduce themselves. Once one is made, no additional fabrication is required 


\section{Nanobioengineering}

Nano-engineering of a biological system deals with the assembly of nanomaterials to create or enable a specific biological function and/or the subsequent characterization of that function. This can be viewed as separate from the study of the nano-biomaterials themselves, which is defined by their isolation and characterization or their synthesis from basic building blocks [10-12].

The extension of the field of nano-engineering to biology includes the patterning of 2D templates to direct cell or tissue response for biocompatability or biosensor applications. Applications in medical science include implants, prosthesis, drug delivery and diagnostics. Other examples include creating a biological input/output device using nano-fabrication techniques to enable communication with individual cells for information technology applications. The field of computer science is struggling with the problem of how to integrate information science with biology. One approach has been through bioinformatics, where traditional computer science methodology and hardware is applied to manage the enormous amount of information now available from biotechnology. Another approach envisions the integration of the biology with computer science to create new hardware to enable direct communication with the biology. If successful, this will provide the platform to allow the treatment of biology as just another peripheral for functions such as sensing, data storage and information security. Other applications of this hybrid system could be communication with individual cells to switch on and off biomanufacturing of drugs in vivo, or the construction of biological-nonbiological hybrids for robotics study and application. Several basic research directions seem promising to enable fabrication of such devices:

The primary objective should be the Nanoengineering of the Biological Interface [13]. The success of two-way communication strategies between electrically active cells and microelectronics depends on the proper registration of cells with the microelectrodes and their close association or "seal" to those microelectrodes [14]. The "seal resistance," $\mathrm{R}_{\text {seal }}$, is measured between the electrode and the grounded recording media. Low values of $\mathrm{R}_{\text {seal }}$ are associated with poor electrode-to-cell adhesion resulting in an attenuated and distorted bioelectrical signal, a problem inherent in most extra-cellular recording systems. In contrast, $\mathrm{R}_{\text {seal }}$ values in the giga-ohm range, which is typically achieved using glass micropippettes during electrophysiology measurements, permit clearer resolution of bioelectric signals. In tissue this seal is accomplished by the interactions of proteins and other biological macromolecules with the glycocalyx, or outside surface, of the cells. The first step in recreating this interface is nanoengineering of the non-biological surface with biologically compatible materials such as proteins, peptides and biologically active functional groups. Surface modification allows a large variety of biomolecules to be attached to a microelectrode surface which will facilitate cell-microelectrode adhesion, increase $\mathrm{R}_{\text {seal, }}$, and allow for geometric placement of cells [15]. For example, it is possible to control the nutrients fed to cells so that they produce desirable surface groups that allow binding of those cells to specific nonbiological surfaces [16]. The immediate benefit is that if a cell is associated with a single microelectrode, it becomes an individual sensor permitting multiple independent assays that then enable statistical analysis. Extracellular recording of these individual sensor elements would then allow long-term, multi-site measurements of electrically-active cell bodies and processes for building information technology devices.

Another major focus area is the creation of Hybrid Cellular/Electronic Control Systems. The goal is to develop the electronic/neuronal interface and then use this to control a biological transducer to study robotics applications. Developing this system will demonstrate a new strategy for controlling a simple cellular/mechanical/electrical hybrid robotic system. Simple model systems could provide information as to what hardware and software enable truly adaptable, coordinated, and graceful systems for movement and problem solving. One example of a hybrid model system is the spinal stretch reflex arc, one of the most fundamental neuronal feedback circuits in the nervous system. Duplication of just this system would increase robotic capability enormously. 
- Living systems can be manipulated. We can specifically alter the genetic blueprint, chemically modify the individual molecules, and in fact now even alter the genetic code itself.

- Living systems can be components of hybrid structures-part living, part synthetic, incorporating selected properties of both as required.

- $\quad$ Living systems are, in the absence of immunological rejection, biocompatible.

\section{Future Directions}

Each of these characteristics or concepts can be regarded as a target property of nanostructured materials and devices. The effort to achieve this must be multi-disciplinary. The goals of the biologist are to identify desirable functions performed by living systems and to work to understand how they are performed. That knowledge, used by chemists, physicists and engineers would then lead to the development of nanoscale systems that use those techniques to achieve similar required functions.

The nature of the materials selected to achieve this, and their organization and architecture is left to the imagination of scientists who will be drawn to these challenges. They will use their particular background, coupled with that of their collaborators from other fields, as the basis for proposing novel approaches to these problems. Recreating these properties is in many cases a difficult challenge, but the rewards are incalculable. It is a multi-decade program of a broad range of research, but it is one that will provide breakthroughs of great significance almost from the start.

\section{References}

1. D. Charych, Q. Cheng, A. Reichert, G. Kuziemko, N. M. Stroh, J. O. Nagy, W. Spevak, and R. Stevens, "A 'Litmus Test' for Molecular Recognition Using Artificial Membranes," Chemistry and Biology 3, 113 (1996).

2. E. Greenbaum, "Platinized Chloroplasts: A Novel Photocatalytic Material," Science 230, 1373 (1985).

3. H. Noji, R. Yasuda, M. Yoshida, K. Kinosita Jr., "Direct Observation of the Rotation of $F_{1}-$ ATPase," Nature 381, 623 (1996).

4. T. Honjo, and S. Habu, "Origin of Immune Diversity: Genetic Variation and Selection," Annual Review of Biochemistry 54, 803 (1985).

5. X-D. Xiang, and P. G. Schultz, "The Combinatorial Synthesis and Evaluation of Functional Materials," Physics C 282, 428 (1997).

6. D. G. Nicholls, and S. J. Ferguson, "Bioenergetics 2," Academic Press (1992).

7. B. Sakmann, "Elementary Steps in Synaptic Transmission Revealed by Currents Through Single Ion Channels," Science 256, 503 (1992).

8. D. E. Koshland Jr., "Chemotaxis as a Model Second Messenger System," Biochemistry 27, 5829 (1988).

9. C. Walsh, W. H. Freeman, Enzymatic Reaction Mechanisms p. 33, (1979). 
10. V. Stupp, LeBonheur, K. Walker, L. S. Li, K. E Huggins, M. Keser, and A. Amstutz, "Supramolecular Materials: Self-Organized Nanostructures," Science 276, 384 (1997).

11. S. I. Stupp, and P. V. Braun, "Molecular Manipulation of Microstructures: Biomaterials, Ceramics, and Semiconductors," Science 277, 1242 (1997).

12. D. A. Hammer and M. Tirrell, "Biological Adhesion at Interfaces," Annu. Rev. Mater. Sci. 26, 651 (1996).

13. J. J. Hickman and D. A. Stenger, "Interactions of Cultured Neurons with Defined Surfaces," in: Enabling Technologies for Cultured Neural Networks (D.A. Stenger and T.M. McKenna, eds.), 1st edition. San Diego: Academic Press, Inc. 51 (1994).

14. D. S. Jung, J. J. Cuttino, P. Pancrazio, T. Manos, R. S. Custer, L. E. Sathanoori, M.G., Coulombe, M. A. Czarnaski, D. A. Borkholder, G. T. Kovacs, P. Bey, D. A. Stenger, and J. J. Hickman, "Cell-Based Sensor Microelectrode Array Characterized by Imaging XPS, SEM, Impedance Spectroscopy, and Cellular Recordings," J. Vac. Sci. Tech. A 16, 1183 (1998).

15. M. S. Ravenscroft, K. E. Bateman, K. M. Shaffer, H. M. Schessler, D. R. Jung, T. W. Schneider, C. B. Montgomery, T. L. Custer, A. E. Schaffner, Q. Y. Liu, Y. X. Li, J. L. Barker, J. J. Hickman, "Developmental Neurobiology Implications from Fabrication and Analysis of Hippocampal Neuronal Networks on Patterned Silane-Modified Surfaces," J. Amer. Chem. Soc. 120, 12169 (1998).

16. K. J. Yarema, L. K. Mahal, R. Bruehl, E. C. Rodriguez, and C. R. Bertozzi, "Metabolic Delivery of Ketone Groups to Sialic Acid Residues: Application to Cell Surface Glycoform Engineering," J. Biol. Chem., 273, 31168 (1998). 


\section{HYBRID SYSTEMS: INTEGRATING FUNCTIONALITY}

Nanotechnology exploits the new properties, phenomena, processes, and functionalities that matter exhibits at sizes intermediate between isolated atoms or molecules $(\sim 1 \mathrm{~nm})$ and bulk materials (over $100 \mathrm{~nm}$ ). Nature abounds with examples of nanoscale structures that are used to provide critical functions including mechanical actuation (muscles), energy conversion (photosynthesis), and sensing and molecular recognition (olfactory centers).

The organization of artificial nanoscale structures has been achieved by taking advantage of inherent molecular interactions including van der Waals, hydrogen bonding, electrostatic, or covalent bonding forces that promote spontaneous self-assembly. Using this approach, nanoscale assemblies that demonstrate extraordinary optical [1], mechanical [2], and sensing [3] functions have been developed. Unfortunately though, the key requirement of addressability, the ability to direct the movement of energy or signals within a useful architecture, is a more difficult challenge than can be accomplished using the tools of self-organization alone.

Biological analogues suggest that the ideal architectures for exploiting nanoscale structures will be hierarchical, spanning from the nanoscale to the microscale and perhaps even human dimensions. By combining the best features of both "top-down" and "bottom-up" strategies it is possible to exploit the unique capabilities provided by nanotechnology in useful and addressable machine architectures. Since the top-down approaches, based on various forms of lithography and replication, now extend to approximately $100 \mathrm{~nm}$, we have the opportunity to form a seamless transition between nano- and microtechnology.

\section{A. Current Status and Recent Accomplishments}

Researchers are currently exploring approaches that integrate unique functions achieved through nanotechnology into microscale devices. As an example, consider miniaturized instruments for chemical and biomolecular analysis. This will be an area of intense research with strong impact likely on health, environment, and national security.

Microfabricated chips for DNA analysis [4,5] and for polymerase chain reaction [6] have already been demonstrated. These are perhaps the initial steps towards a full-fledged technology of biomedical microdevices, which will not only study and analyze nucleic acids but other biological molecules such as proteins, carbohydrates etc.

The development of chips for rapid detection of biological pathogens is a critical area with applications in the food handling/processing industry, in biological/chemical warfare, and in early warning for exposure to air and water-borne bacteria, viruses, and other antigens. The $\mu$ ChemLab project [7] is learning to incorporate similar structures into a fully autonomous chemical analysis system that is integrated into on-chip architectures. For example, organically functionalized meso-porous structures have been integrated on a micromachined heating and 
flow stage to provide 1000-fold chemical pre-concentration for on-chip analysis of chemical warfare agents.

Thundat et al. [8] recently demonstrated that the interaction of antigen molecules with their corresponding antibody, attached to the surface of a cantilever, can provide sufficient surface stress to bend the cantilever beam (see Fig. 8.1). Such a device is an example of hybridization and integration at several levels, because it has nanocomponents (antibodies bound to a cantilever surface) and microcomponents (cantilever beams) which can be delivered in a chip (millimeter scale components) that together integrate biology and biochemistry with engineering.

Boxer [9] recently demonstrated the deposition of lipid membranes into lithographically defined corrals. Early results suggest that this approach may allow electrochemical addressing of photo-defined membrane cells. Consequently, this is a first step toward integrating the functional nanotechnology demonstrated by living cells into robust machine architectures.

The design and realization of specific structures at near-atomic scales requires controlling materials according to prescribed macro- and meso-scopic specifications, which are actually decided at the quantum level. Despite recent advances, a number of outstanding problems remain to be solved (see box on "Control Strategies at the Nanoscale").

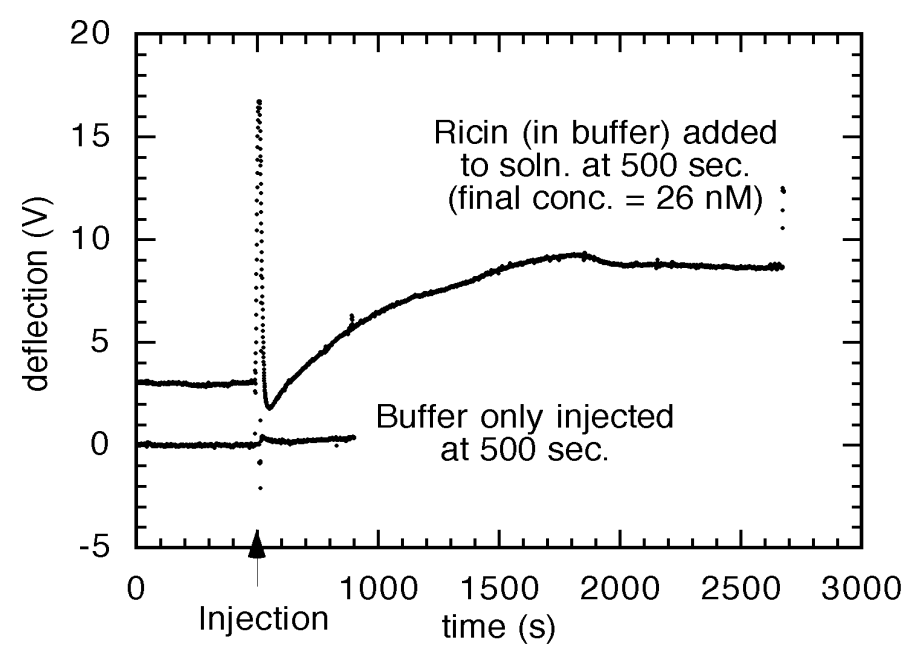

Figure 8.1: Chemomechanically induced cantilever deflection by ricin antigen-antibody $(\mathrm{Ab}-\mathrm{Ag})$ reaction. The bottom curve shows absence of deflection when $\mathrm{Ab}$-loaded cantilever is exposed to a buffer solution, i.e. without containing ricin Ag.

\section{B. Key Questions and Barriers}

1. The processing conditions for "top-down" (lithographic) and "bottom-up" (nanoscale) manufacturing can be quite different and may create thermal and chemical incompatibilities. For them to seamlessly mesh we will need to better understand how to improve the stability of nanostructures and to modify or find new patterning approaches.

2. The unique properties associated with nanoscale structures and materials can be dramatically influenced by the dominance of interfaces on the nanoscale. We will need to understand how to tailor and control interfaces that maintain and potentially enhance the performance of nanoscale functions and properties. 


\section{Control Strategies at the Nanoscale}

Experimental and technical advances now make it possible to design and realize specific structures at near-atomic scales. This process requires controlling materials and structures according to prescribed macro/mesoscopic specifications, which are actually decided at the quantum level over nanometer spatial scales [10-19]. Despite notable advances, outstanding problems remain to be solved, namely (i) deeper theoretical understanding of new emerging properties from the purely quantum description; (ii) reproducible control techniques at the nanoscale; and (iii) high throughput, reliable assembly at industrial scale. Research on efficient control techniques at the nanoscale will span the following directions.

Optimal Control. Within the optimal-control formalism, the "goal" (the desired effect) and "cost" (the effort needed to achieve it) are combined into an objective functional. This functional is maximized under the dynamical constraint that the Schrodinger equation describing the underlying system be satisfied. The quantum control problem differs from the traditional engineering control algorithm in that the wave function is not available to observation. Therefore, one must construct the control functional in terms of those functionals of the wave function that can be measured, and which will be used in the control algorithm as constraints. From these global quantities, one tries to obtain local quantities, such as the potential as a function of position along the mesoscopic structure. Since several combinations of adjustable parameters may yield similar results, the determination of the "cloning" potential may not be unique. However, for practical applications the main issue is not uniqueness, but easy feasibility and robust reproducibility $[13,19]$.

Coherent Control. The coherence of laser light has been used to guide quantum systems into desired target states through interfering pathways. The measured shape of the wave function is used as feedback into the laser, which reprograms the optical field. The process is iterated until the shape of the wave function matches the target wave packet. If successful, the ability to control the shape and motion of the wave function may lead to efficient methods for bond-selective chemistry and quantum computing. Laser technology has advanced in regimes of frequency, pulse duration and shape, and peak intensity so that concepts of coherent control may be applied to individual atoms. The current state-of-the art in solid-state lasers operating at wavelengths near $1000 \mathrm{~nm}$ has yielded pulses as short as $5 \mathrm{fs}$ and intensities of $10^{22} \mathrm{~W} / \mathrm{cm}^{2}$, well in excess of the atomic unit of intensity. Under these extreme conditions, new physical phenomena appear, as substantiated by the steady flow of results on multiphoton ionization and highharmonic generation. In such phenomena, the external field is not a small perturbation, but significantly shapes or distorts the quantum structure and dynamics. Individual states of the atom in the field experience large dynamical energy shifts, and may even cross energy thresholds, disappearing from the spectrum, only to be replaced by other "light-induced" states (LIS). Applying coherent control to the quantum evolution of the atom-laser system, unique phenomena, such as the stabilization of atoms against ionization, may be exploited. (Atomic stabilization is the "counter- intuitive" trend by which, at some high intensity, the ionization rate decreases with increasing intensity.) The sustained, controlled exposure of atoms to super-high-intensity radiation will extend the application of current high-harmonic generation techniques. The question of what happens to harmonic emission of radiation in the stabilization regime of ionization or the role of the LIS, are open questions that require more research $[10,14,16]$.

Statistical Control. To produce, verify, and diversify nanomaterials and nanosystems at industrial scale, for specific requirements and customers, while maintaining high throughput and high economic efficiency, will require implementing a robust and efficient form of statistical control.

New Control Paradigms. It will be profitable to shift the current "chisel down" approach to nanostructures to a "grow up" approach based on molecular self-assembly and/or biological assembly, self-replication, self-aligned quantum dots structures, and bioarray technologies. The challenges of this approach are related to: combinatorial searches, kinetics in self-assembly, evolvable hardware, autonomous reprogramming, bioassembly, and related topics. 
3. The integration of nanoscale and microscale functionalities will provide new opportunities that are not reflected in simple miniaturization of macroscopic device analogues. We will need to step away from conventional thinking about device architectures in order to better exploit the real benefits of nanotechnology.

\section{Future Research Directions}

The technology for microelectronic, optoelectronic, and magnetic devices is well established. They interact with their environment in either one or two ways, i.e. either purely electrically or optoelectronically or else magneto-electronically etc.

One can imagine a wide variety of other devices that will interact differently with their environment. These interaction mechanisms include but are not restricted to: chemically (sensors), biologically (sensors, analyzers, drug delivery), thermally (engines, refrigerators), acoustically (sensors and actuators), and mechanically (MEMS devices). In the future, it is expected that a technology for truly hybrid devices will be developed that will be not only multifunctional but inexpensive. Hybridization will occur in scale as well as interactions. Such devices will contain components ranging in size from nanometer-scale (biomolecules, nanocrystals etc.) to micrometer-scale (cantilever beams, electronic interconnects) and to millimeter-scale (packaging/handling).

In addition to hybrid devices that provide new functions and capabilities, the integration of nanoscale and microscale functionalities will provide new means to directly study nanoscale phenomena. For example, micromachined scanning-probe instruments are creating new means to image and measure a wide range of properties of nanoscale materials and structures. The addition of nanoscale structures such as carbon nanotubes, to the probe itself, holds promise for even greater resolution and functional capability. (See "Nanoscale Instrumentation" in this report.)

\section{References}

1. T. J. Marks, M. A. Ratner, Angew. Chem. Int. Ed. Engl. 34, 155 (1995).

2. H. Noji, R. Yasuda, M. Yoshida, K. Kinosita, Nature 386, 299 (1997).

3. X. Song, J. Nolan, B. I. Swanson, J. Am. Chem. Soc. 120, 11514 (1998).

4. B. Lemieux, A. Aharoni, M. Schena, "Overview of DNA chip technology," Molecular Breeding 4, 277-289 (1998).

5. K. M. Kurian, C. J. Watson, A. H. Wyllie, "DNA chip technology,” J. Pathology 187, 267271 (1999).

6. M. U. Kopp, A. J. deMello, A. Manz, "Chemical amplification: Continuous-flow PCR on a chip," Science 280, 1046-1048 (1998).

7. Frye et al., 477 in "Micro Total Analysis Systems '98" Ed. Harrison and van den Berg Kluwer Aced. Press (1999).

8. T. Thundat, P. I. Oden, R. J. Warmack, "Microcantilever sensors," Microscale Thermophysical Engr. 1, 185-199 (1997).

9. J. Groves, N. Ulman, S. G. Boxer, Science 275, 651 (1997).

10. W.S. Warren et al., "Coherent control of quantum dynamics: the dream is alive," Science 259, 1581 (1993). 
11. D. Bessis et al., "Electron wave filters from inverse scattering theory," Europhys. Lett. 37, 151 (1997).

12. T. Seideman, "Molecular optics in an intense laser field: a route to nanoscale material design," Phys. Rev. A. 56, R17 (1997).

13. A. N. Zakhariev and V. M. Chabanov, "New situation in quantum mechanics (wonderful potentials from the inverse problem)," Inverse Problems 13, R47 (1997).

14. D. Meshulach and Y. Silberberg, "Coherent control of two-photon transitions by femtosecond laser pulse," Nature 396, 239 (1998).

15. A. Bulatov et al., "Nonadiabatic cooling and optimal control in off-resonance dipole optical potentials," Phys. Rev. A 58, 1346 (1998).

16. N. Bonadeo et al., "Coherent optical control of the quantum state of a single quantum dot," Science 283, 1473 (1998).

17. R. B. Perez, V. Protopopescu, and L. Munoz-Cobo, "Control of quantum systems," Math. Models and Math. in Appl. Sci. 9, 305 (1999).

18. T.C. Weihnacht, J. Ahn, P.H. Bucksbaum, "Controlling the shape of a quantum wavefunction," Nature 397, 233 (1999).

19. G. Harel and V. M. Akuli, "Complete control of Hamiltonian systems: engineering of Floquet evolution," Phys. Rev. Lett. 82, 1 (1999). 


\section{NANOSCALE INSTRUMENTATION}

Progress in nanotechnology requires having the tools to observe, characterize, and control phenomena at the nanometer scale. A whole new generation of analytical instrumentation and nanoscale devices, capable of providing information about nanoscale physical, chemical and mechanical phenomena must be developed. Nanotechnology is already benefiting from novel instrumentation developed during the past two decades, for example scanning probe microscopes and a new generation of synchrotron $\mathrm{x}$-ray sources capable of studying materials at the nanoscale. On the other hand, discoveries in nanoscale science and engineering can provide the basis for the development of unprecedented new tools. Thus, the next decade will provide both opportunities and challenges in developing instruments for synthesizing nanostructures as well as for characterizing nanostructures and measuring properties at the nanoscale.

\section{Recent Advances and Current Status}

Several recent advances in the national laboratories are contributing to the development of new nanoscale instrumentation. Rapid progress in micro-electromechanical systems (MEMS) is opening new directions in materials research at small length scales. For example, researchers have developed a MEMS strain gauge from nanocrystalline diamond as seen in Figure 9.1 [1].
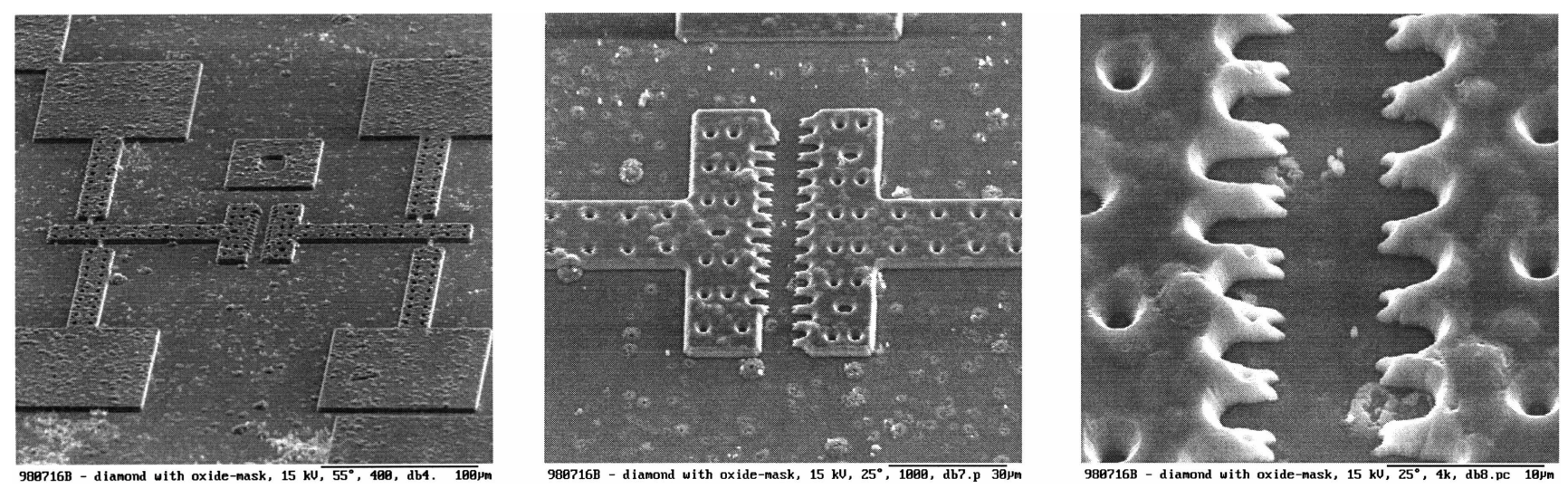

Figure 9.1: SEM micrograph of the vernier indicator of a nanocrystalline diamond strain-indication structure. The four anchor pads are on the Si substrate, and the vernier scales and arms are deposited on $\mathrm{SiO}_{2}$. The 2- $\mu \mathrm{m}$-thick diamond indicators and support arms are detached from the substrate by a gas-phase $\mathrm{CF}_{4}$ etch (Ref. 1).

Understanding an atom's travels over a surface at different temperatures, as well as its ultimate incorporation into the surface, is crucial to making smaller, faster, smarter devices. A new instrument called the "Atom Tracker" [2] now can observe an atom in motion. The Atom Tracker supplements the STM's vertical feedback with lateral electronic feedback to keep the probe hovering over the atom of interest, and can track atomic motions 1000 times faster than a conventional STM. By continuously monitoring the motion of an individual atom as it binds to various sites on a surface the diffusing atom itself becomes a probe of the surface structure and properties. 
An electron-beam tool based on reflection electron holography performed at ultra-low beam energies is being developed for critical dimension (CD) and defect review metrology [3]. The target resolution limit is $0.5 \mathrm{~nm}$ at $100 \mathrm{eV}$ energy, permitting the system to perform CD metrology for devices built to design rules well below $10 \mathrm{~nm}$ (i.e. the device generation currently projected for 2009). The molecular level of resolution and the low beam damage resulting from the choice of beam energy make this tool ideally suited for emergent nano-technologies including purly biological or hybrid device structures.

In order to understand processes used to produce nanometer-sized particles deposited on surfaces, new aerosol instrumentation is needed. Significant progress has been made recently, with instruments now available for detecting particles as small as several nanometers in diameter [4,5]. Mobility classifiers that enable high-resolution classification according to particle size also have recently been reported [6,7]. These instruments operate at gas pressures in the range of one atmosphere, while measurements of nanoparticle size distributions at pressures down to 0.1 Torr are possible with a particle-beam mass spectrometer [8].

The inventions of the scanning tunneling microscope (STM) [9] and the atomic force microscope (AFM) [10] have spawned the development of a variety of new scanning probe microscopes (SPMs) [11]. Whereas the STM and AFM have found applications mainly in topographical imaging, the other SPMs add more functionality. Examples include the near-field scanning optical microscope (NSOM) [12], scanning thermal microscope (SThM) [13,14], scanning capacitance microscope (SCM) [15], magnetic force and resonance microscopes (MFM) (see Figure 9.2) [16,17], and the scanning electrochemical microscope (SECM) [18]. By providing access to and enabling observation of physical, chemical, and biological phenomena at nanometer scales, these SPMs have changed the landscape of nanoscale scientific research.

A sensor that can measure the atomic-level forces that develop between two surfaces as they approach each other and come into contact also has been developed [20]. The Interfacial Force Microscope (IFM) utilizes a feedback force sensor that eliminates the snap-to-contact event that is inherent in other scanning force microscope designs. The IFM allows measurement of the full range of adhesive interactions and can be used to image surfaces by controllably hovering out of range of contact.

Just as scanning probes have revolutionized our ability to image and characterize nanoscale structures, it is clear that the ability to explore multiple functions with a single probe (multitasking scanning probes) will bring about a new revolution. For example, the combination of a force microscope with a scanning near-field optical microscope was recently demonstrated [21]. This new capability allows one to directly image and correlate changes in molecular conformation, as indicated by specific optical transitions, with the mechanical compliance of molecules. 

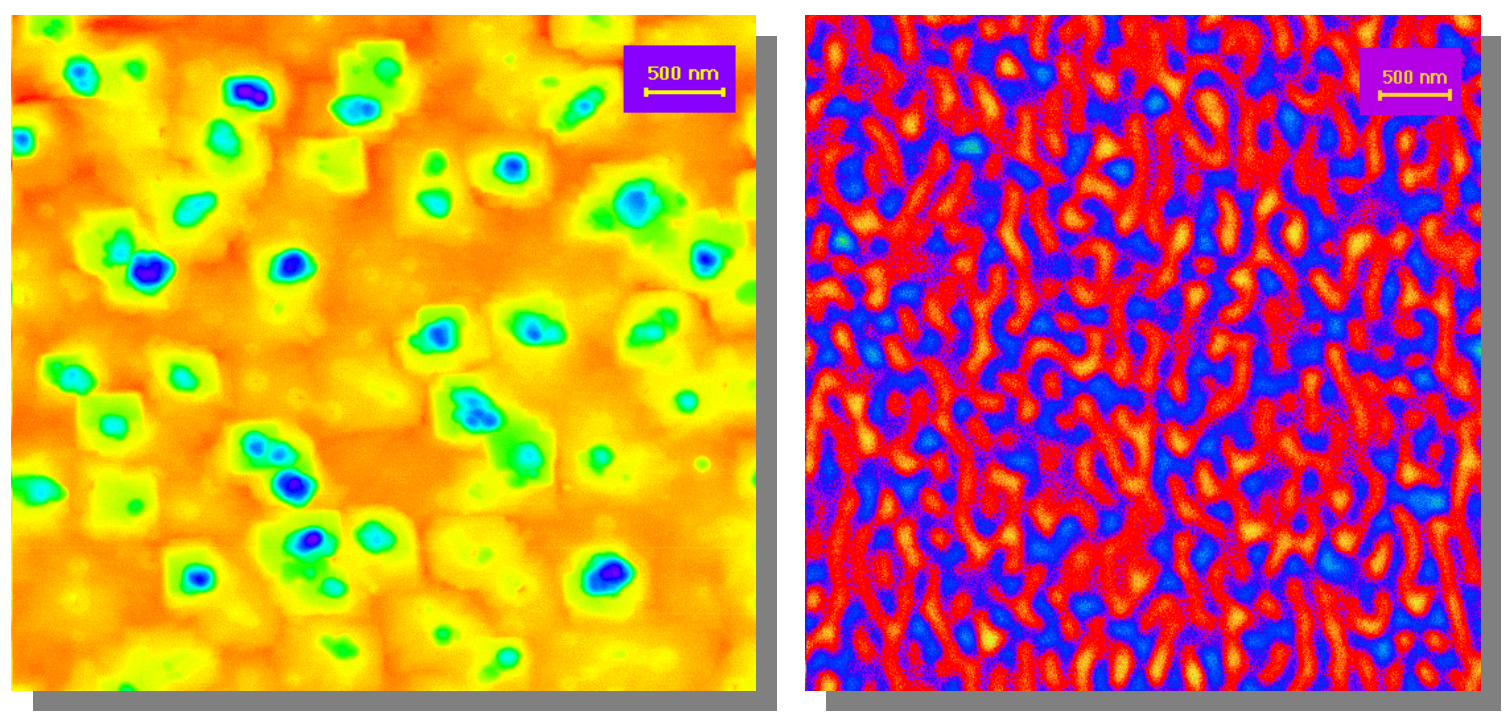

Figure 9.2: AFM (left) and MFM (right) images of a Sm-Fe film grown epitaxially by sputter deposition. The MFM image shows stripe domain patterns characteristic of magnetic films with strong perpendicular magnetic anisotropy. The epitaxial Sm-Fe film lacks domain wall pinning and is an ideal candidate base material for creating artificial pinning sites. The information provided by studies of domain wall-defect interaction would greatly benefit research on permanent magnet materials. (Ref. 19).

Recent advances in laser desorption mass spectroscopy have made possible real-time detection, sizing and chemical analysis of individual aerosols larger than $200 \mathrm{~nm}$ [22,23]. It is possible to develop a versatile system making use of various interfaces which deliver intense beams of sizeselected particles from ambient conditions into a vacuum chamber for analysis. The combination of differential mobility analyzers with such a system will make possible sizing and chemically characterizing individual aerosols in real-time.

Current $\mathrm{x}$-ray capabilities at third-generation synchrotron sources such as the Advanced Photon Source, as well as neutron-scattering capabilities being developed at The Spallation Neutron Source, facilitate new types of in situ studies of the synthesis and processing of nanostructured materials, and will clearly play an important role in establishing structure-property relationships for nanostructured materials. Direct radiation from synchrotron sources already provides x-ray beams with intensities capable of real-time (spatial average) monitoring of the formation, evolution, coating, and encapsulation of nanoparticles on substrates using techniques such as small-angle scattering, large-angle diffuse scattering, and direct Bragg diffraction measurements [24]. The application of significantly submicron focused beams can provide in situ, spatiallyresolved structure and property measurements during synthesis, processing, and physical testing. Real-time $\mathrm{x}$-ray diffraction measurement of solution-injection nucleation, growth, and evolution of thin films and precipitates is presently possible. For example, real-time $\mathrm{x}$-ray scattering techniques have been used recently to investigate the growth modes and surface structure of GaN thin films grown by metal-organic chemical vapor deposition [25,26]. Using microscale (i.e. laboratory on a chip) techniques [27] in combination with micro and nano x-ray beams, highresolution time-resolved measurements can be accomplished in steady state by controlling the stream velocity and varying the distance between the injection point and the measuring position. Scaling measurements to smaller sizes, to more complex geometries, and the use of combinatorial analysis techniques are obvious extensions of microbeam $\mathrm{x}$-ray techniques to the nanoscale. 


\section{Key Questions and Barriers}

A number of questions need to be addressed in developing the next generation of instruments capable of probing structure and properties at the nanoscale. Among these are:

1. Can new techniques be developed to determine local but non-surface structure and properties in composite materials? In such materials the local region of interest is of nanometer-size but is only a small fraction of the total sample volume. While techniques such as X-ray diffraction can provide global-averaged information, and techniques such as AFM can provide nearly atomic-scale information about surface structures and properties, currently only electron microscopy techniques are useful in probing local (nm-scale) nonsurface structures, and there are no techniques at all for investigating properties of nanometer-sized non-surface regions.

2. What materials and materials research are needed to develop the next generation of finescale instrumentation? MEMS devices currently are almost exclusively Si-based. Very short operating lifetimes due to poor friction and wear properties of Si severely limit their potential usefulness. Devices manufactured from alternative materials are clearly needed. One possibility is to develop new MEMS devices based on nanocrystalline diamond [1].

3. How small is too small? The push for miniaturization eventually will reach a quantummechanical limit where, for example, Newtonian mechanics will not be able to adequately predict behavior. Future instruments that manipulate individual atoms will need, for example, to take the Heisenberg uncertainty principle into account.

\section{Future Directions}

Properties of nanomaterials are exquisitely sensitive to details of their atomic structure, 3D shape, surface termination, surface and 'bulk' defects, impurity atoms and locations. New tools are required to map structure, chemistry and functionality, ideally at the atomic level. We need single-atom sensitivity to impurities; 3D shape determination with atomic accuracy; and the ability to find functional sites and determine the origin of that function. It is desirable to measure optical absorption and emission from individual elements, preferably with femtosecond time resolution. These tools will come from the further development of current microscopies including scanning tunneling microscopy, confocal and near-field optical microscopy, atomic resolution transmission and scanning transmission electron microscopy, electron energy loss spectroscopy, cathodoluminescence and electron-beam-induced-current imaging.

Traditionally, improved or new characterization tools have paved the way for increased understanding in materials science, e.g. electron microscopy. The same will be true for nanoscale materials science. Scanning probes already are making a huge impact. But new tools are needed to image and characterize buried interfaces with nanoscale resolution, and these must operate in a wide range of temperature and environments to be of greatest use.

While various forms of microscopy (TEM, SEM, AFM, STM, etc.) can furnish detailed information about morphology, size, and on occasion the composition of nanoparticles, none are capable of providing real-time, on-line information. There is a clear and pressing need for new 
techniques and instruments that allow for rapid detection and for chemical and physical characterization of nanoparticles, as well as for automated or quasi-automated systems that require a minimum of operator attention.

With the STM atoms can be manipulated [28], but only at low temperatures. We can manipulate molecules at room temperature but only two examples have been shown to date [29,30]. With scanning probes, we can fabricate nanoclusters containing under 10,000 atoms and nanowires less than $4 \mathrm{~nm}$ wide with great control, but at an unacceptable cost in time. The metallicity of a cluster can be determined with scanning tunneling spectroscopy, but we cannot determine its chemical identity or its magnetic properties. To address these shortcomings will require a broad program of technique and instrumentation development.

A major effort also must be devoted to invent communication and networking devices that operate at the nano-scale. The underlying methods and technologies may be targeted at noninvasive exploitation of indigenous media, and should include capabilities for interfacing with operators and other macro-scale devices in the outside world.

Scanning probe microscopes (SPM) are limited to analyzing surface or near-surface properties of solids with nanometer-scale spatial resolution. Sub-surface imaging and truly 3D microscopy with nanometer-scale resolution is not currently available but is extremely important for future development of nanotechnology. For example, most biological nanostructures are 3D and are currently imaged by $\mathrm{x}$-ray crystallography, which is expensive and time consuming. Even in micro/nano-electronics, which is progressing toward 3D multi-layer structures, 3D imaging would be very useful. Possible approaches for sub-surface imaging include ultrasonic echo imaging, non-linear (multi-photon) optical microscopy, or thermal spectroscopic imaging. It is unclear at present which technique will be most suitable, but this area of investigation needs to be emphasized.

Nano-sensors have a high potential for deployment in areas and media that are not readily amenable to probing by traditional devices. An example is medical operations requiring minimally invasive surgery. A collection of specialized devices may be needed to provide all of the necessary functionalities. One can imagine an ensemble of micro or nano-robots that cooperatively explore, assess, and perhaps operate on various locations in an organ. This poses formidable challenges in signal processing, control, and interconnectivity. (See box on "Networks of Nano-Sensors.")

The realization of new technologies based on nanostructured materials is limited in part by our ability to develop and control materials processing to achieve desired properties. However, revolutionary developments in $\mathrm{x}$-ray focusing optics, in connection with ultra-high brilliance third-generation synchrotron $\mathrm{x}$-ray sources, should produce rapid advances in materials probe capabilities that will complement TEM and SPM capabilities. Fresnel zone plates have been used to demonstrate intense, focused beams of hard $\mathrm{x}$-rays with resolution below $100 \mathrm{~nm}$ at the Advanced Photon Source [31,32] and hard $\mathrm{x}$-ray beams down to the $20 \mathrm{~nm}$ range now appear within reach. These $\mathrm{x}$-ray probes are complementary to the many powerful scanning probe and scanning electron microcopies in that they provide non-contact, nondestructive access to the depth dimension in harsh environments, and both physical properties and atomic structure information is available. (See box on "Synchrotron X-rays for Process Discovery and Control in Gaseous Environments"). Nanobeam $\mathrm{x}$-ray applications in the $10^{2}-10^{3} \mathrm{~nm}$ resolution range are 


\section{Networks of Nano-Sensors}

Conventional networking methods are inadequate for ensembles of nano-sensors and micro- or nano-robots because of limitations of size as well as the medium in which the devices may be deployed. Optical communication is not possible in opaque media nor is electromagnetic propagation possible in electrically conducting liquids. New methods are needed to exploit the native medium to communicate between sensors with minimal invasion. One example is to use tiny sonic bursts to communicate between sensors deployed in fluid or other media. Nanocommunication devices themselves have to be developed to meet limitations of size and power. Interconnectivity among nanosystems and $\mathrm{I} / \mathrm{O}$ with the macro world also are primary research challenges. Clearly, a major effort will be required to invent nanoscale communication and networking devices. Several research topics are illustrated here.

Transmitting and receiving devices. Seeking suitable transmitting and receiving device candidates is expected to be a major part of future research. Both electronic and optical devices have significant merits for communication. Optical devices have an advantage in speed and larger bandwidth and in higher radiated power. Combining nonlinear elements into arrays [38] may significantly enrich the performance of the communicating device. Single nonlinear devices (transmitters and receivers) produce only temporal waveform characteristics of the transmitted/received signal. Arrays of coupled devices will introduce an important additional characteristic to the communicating signal, namely a spatial waveform. Coupling the spatial with the temporal dimension will enhance the ability to decode and encode information for communication and position location.

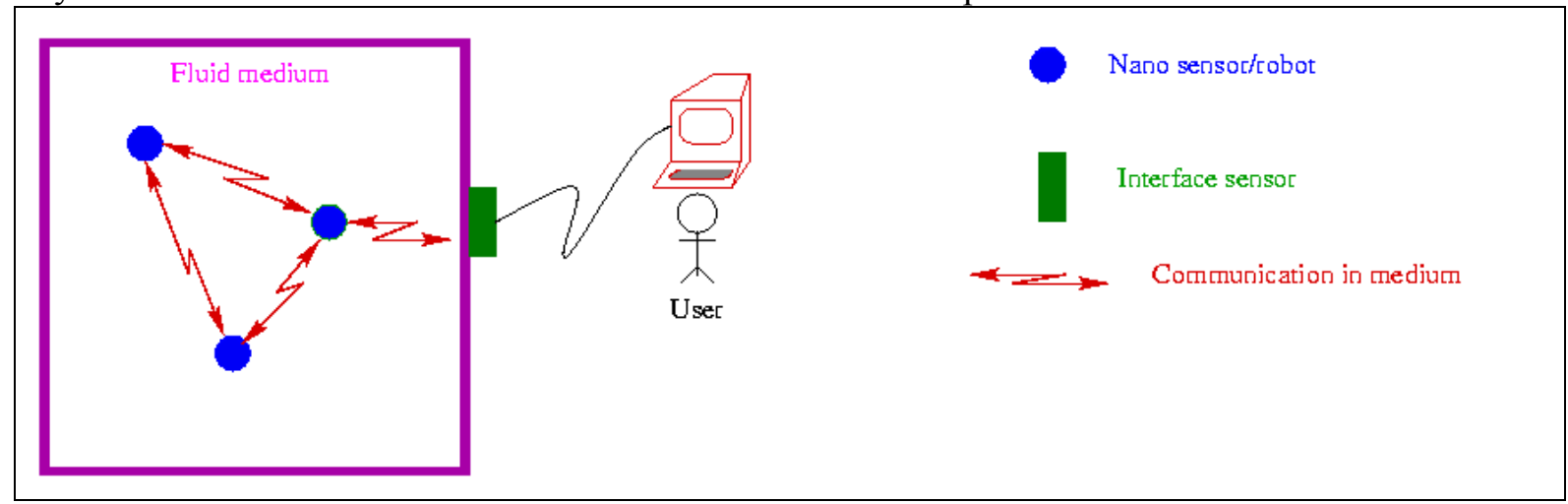

Schematic representation of a nanosensors / nanorobots network

Chaotic Communication. This new communication paradigm, in which the transmitted signal is chaotic, promises greater efficiency, greater number of channels, higher security, and potentially low cost $[39,40]$. The outstanding issue in chaotic communication is synchronization between the transmitter and the receiver [41-44]. Better ways must be identified for robust synchronization between devices and for synchronization of the oscillations in the same device. In addition to already established directions to synchronize chaotic systems [45] new and counterintuitive approaches such as using thermal noise [46] and quenched disorder [47,48] to synchronize and control chaotic systems should be explored. Using noisy and disordered arrays should lead to large savings in fabrication costs.

Biomimetic Communication. Here the goal is to adapt information processing techniques used in biological systems (such as the heart and/or brain) to enhance the capacity of sensing. The key issues requiring study are the ability to communicate by structures and to synchronize oscillations [49,50]. Communication paradigms exhibited by biological oscillators (both experimental and theoretical) may lead to pioneering ways of sensing, detection and transmission of information, and may contribute insights as to how to mimic the communications and interconnectivity of biological systems in man-made nanosystems.

Computational components. Research on communication at the nano-scale should be highly interdisciplinary, since it probes the foundations of physics, chemistry, biology, mathematics, electrical and optical engineering, and computer science. Computational modeling and simulations to interpret and predict experimental findings are essential. Verifying the validity of computational models describing nonlinear nanoscale elements, as well as the efficiency and speed of performing the computations, also will be crucial issues. 


\section{Synchrotron X-rays for Process Discovery and Control in Gaseous Environments}

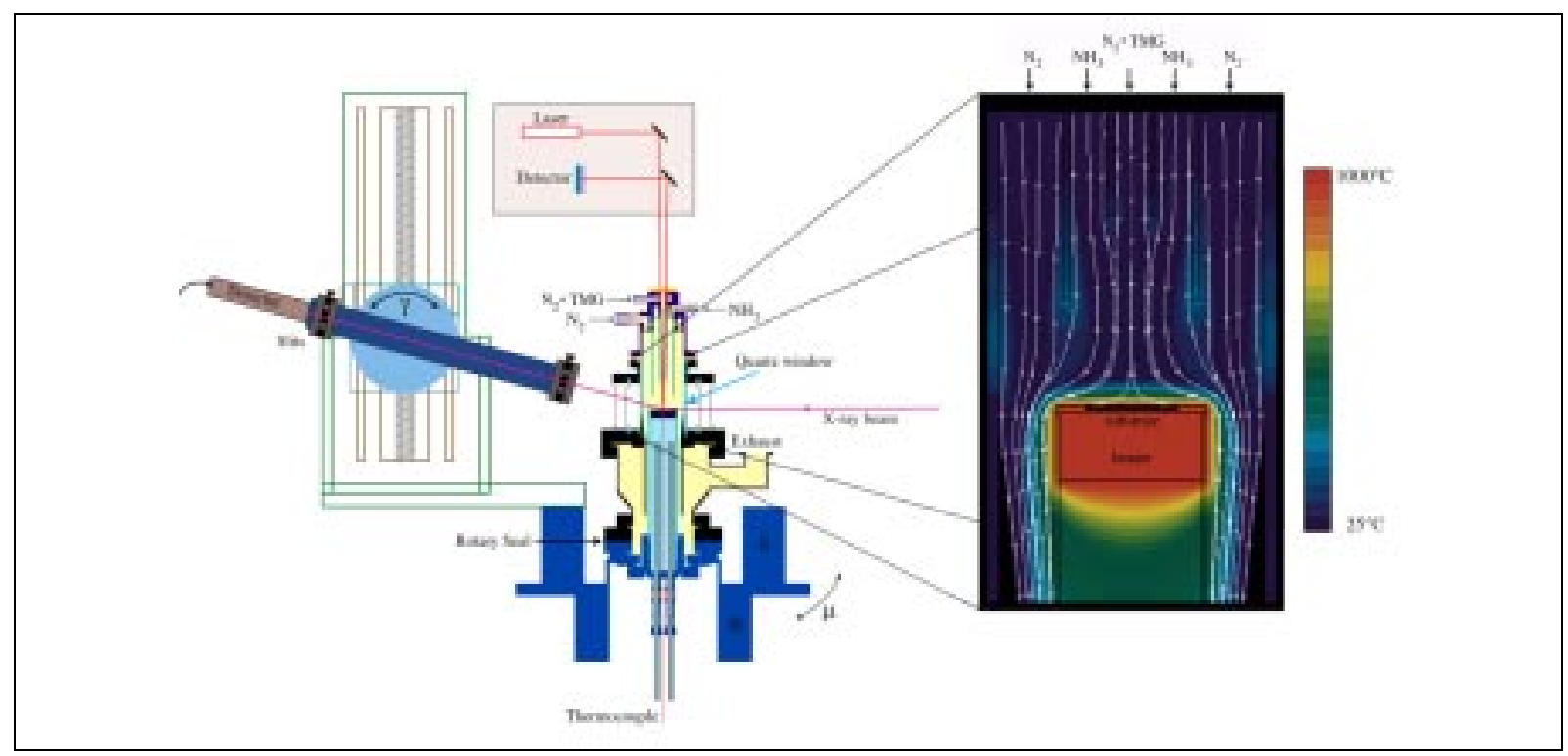

Schematic of the MOCVD real-time processing facility at the Advanced Photon Source. While currently used in studies of epitaxial growth of GaN, such a system is well-suited for future studies of MOCVD processing of oxide and nitride nanometer-scale thin-film heterostructures. The flow patterns and temperatures shown at the right of the figure are the results of unpublished calculations by R. Venkataramani and K.F. Jensen [MIT]. (Ref. 25)

Nanoscale heterostructures are being produced for increasingly complex multicomponent and multilayered oxide films. These oxide multilayers have great potential for device applications. But a major challenge to realize their potential lies in developing in situ diagnostics to control film thickness and nanostructure, including grain size, phase, composition, defect density, and homo- and hetero-phase interfacial properties. Because oxide-film synthesis usually takes place in non-vacuum environments, process monitoring using traditional electron-beam methods is difficult or impossible. In order to provide needed atomic-scale structural and chemical information in oxygen and other gaseous environments, real-time synchrotron x-ray diffraction (SXRD) techniques are currently being developed at the Advanced Photon Source by two national laboratory groups [25,26,33,34]

One group's research seeks to provide scientific understanding of the many coupled phenomena occurring during MOCVD processing of thin films. Current work is focused on $\mathrm{GaN}$ thin film growth behavior and surface structure. Future plans include studies aimed at understanding the physics of ferroelectric domains, particularly in ferroelectric capacitors of size comparable to the domain order parameter, i.e., $\leq 500 \mathrm{~nm}$. Their use of SXRD provides sophisticated measurements of atomic-scale structure, interface morphology, composition, phase content, and chemical states during MOCVD growth and processing (see figure) [25,26]. The other group's research exploits the intermittent nature of pulsed-laser deposition, together with the pulsed temporal structure of synchrotron x-ray beams, to experimentally separate the deposition and surface diffusion/aggregation steps in film growth. This makes possible real-time investigations of the non-equilibrium processes associated with the pulsed-deposition and surface-crystallization and relaxation steps of film growth, on time scales ranging from microseconds to seconds [34].

Support for the continuing development of facilities for in situ, real-time structure and property measurements is needed to speed scientific understanding and to develop advanced growth and processing methods for the production of nanoscale heterostructures. 
under development [33] and transfer of these techniques to still smaller length scales can be anticipated. Consequently, as non-destructive, real-time measurements of the physical properties of nanoparticles and nanostructured materials evolve, and as their use to monitor nanomaterials processing is developed, the field of nanobeam $\mathrm{x}$-ray investigations will provide exceptional opportunities for fundamental and applied investigations.

The challenge of efficient production of nanocluster arrays is most reasonably addressed through parallel processing or self-assembly approaches such as buffer-layer assisted growth [35] or strained-layer growth [36]. While current efforts are promising, they will still require a great deal of developmental effort.

The technology for fabricating thermal, optical, magnetic, or molecular probe structures has been demonstrated [37]. However, probes currently are made for an individual function. An important future goal is to make multi-functional probes for "laboratory on a tip," or "nanoscale total analysis." This will require integration of knowledge from engineering, chemistry, physics, and biology.

\section{References}

1. D. M Gruen, A. R. Krauss, and H. G. Bussmann, (1999), personal communication (unpublished).

2. B. S. Swartzentruber, "Direct measurement of surface diffusion using atom-tracking scanning tunneling microscopy," Phys. Rev. Lett. 76, 459 (1996).

3. D. Joy, personal communication (unpublished).

4. M. R. Stolzenburg, and P. H. McMurry, "An Ultrafine Aerosol Condensation Nucleus Counter," Aerosol Science and Technology 14, 48-65 (1991).

5. T. Seto, K. Okuyama, et al., "Condensation of supersaturated vapors on monovalent and divalent ions of varying size," J. Chem. Phys. 107 (5), 1576-1585 (1997).

6. D. R Chen, D. Y. H. Pui, et al., "Design and evaluation of a nanometer aerosol differential mobility analyzer (Nano-DMA)," Journal of Aerosol Science 29 (5-6), 497-509 (1998).

7. J. F. Fernandez de la Mora, L. de Juan, et al., "Differential mobility analysis of molecular ions and nanometer particles," Trends in Analytical Chemistry 17 (6), 328-338 (1998).

8. P. J. Ziemann, P. Liu, et al., "Particle beam mass spectrometry of submicron particles charged to saturation in an electron beam," Journal of Aerosol Science 26 (5), 745-756 (1995).

9. G. Binnig, H. Rohrer, C. H. Gerber, and E. Weibel, "Surface studies by scanning tunneling microscopy," Phys. Rev. Lett. 49, 57-61 (1982).

10. G. Binnig, C. F. Quate, and C. H. Gerber, "Atomic force microscope," Phys. Rev. Lett. 56, 930-933 (1986).

11. H. K. Wickramasinghe, "Scanned-Probe Microscopes," Scientific American 261, 98-105 (1989).

12. E. Betzig, J. K .Trautman, T. D. Harris, J. S. Weiner, and R. S. Kostelak, "Beating the diffraction barrier: Optical microscopy on a nanometer scale," Science 251, 1468-1470 (1991).

13. A. Majumdar, J. P. Carrejo, and J. Lai, "Thermal imaging using the atomic force microscope," Appl. Phys. Lett. 62, 2501-2503 (1993).

14. A. Majumdar, "Scanning Thermal Microscopy," to appear in Ann. Rev. Materials Science (1999). 
15. C. C. Williams, J. Slinkman, W. P. Hough, and H. K. Wickramasinghe, "Lateral dopant profiling with $200 \mathrm{~nm}$ resolution by scanning capacitance microscopy," Appl. Phys. Lett. 55, 1663-1664 (1989).

16. P. C. D. Hobbs, D. W. Abraham H. K. Wickramasinghe, "Magnetic force microscopy with 25 nm resolution,” Appl. Phys. Lett. 55, 2357-2359 (1989).

17. D. Rugar, C. S. Yannoni, J. A. Sidles, "Mechanical detection of magnetic resonance," Nature 360, 563-566 (1992).

18. A. J. Bard, F. R. F. Fan, D. T. Pierce, P. R. Unwin, , D. O. Wipf, and F. M. Zhou, "Chemical imaging of surfaces with the scanning electrochemical microscope," Science 254, 68-74 (1991).

19. J. S. Jiang, A. Inomata, C. H. Sowers, S. D. Bader, J. B.Patel, X. W. Wu, and C. Thompson, personal communication (unpublished) (1999).

20. J. E. Houston, and T. A. Michalske, "Interfacial-Force Microscope," Nature 356, 266 (1992).

21. A. R. Burns, J. E. Houston, R. W. Carpick, and T. A. Michalske, "Friction and Molecular Deformation in the Tensile Regime," Phys. Rev. Lett. 82, 1181 (1999).

22. S. H. Tolbert, and A. P. Alivisatos, Annu. Rev. Phys. Chem. 46, 595 (1995).

23. A. P. Alivisatos, J. Phys. Chem. 100, 13226 (1996).

24. R. Serna, C. N. Afonso, J. M. Gallesteros, A. Naudon, D. Babonneau, and A. K. PetfordLong, Appl. Surf. Sci. 139, 1 (1999).

25. G. B. Stephenson, J. A. Eastman, O. Auciello, A. Munkholm, C. Thompson, P. Fuoss, P. Fini, S. P. DenBaars, and J. S. Speck, MRS Bulletin 24 (1), 21-25 (1999).

26. G. B. Stephenson, J. A. Eastman, C. Thompson, A. Munkholm, O. Auciello, L. Thompson, P. Fini, S. P. DenBaars, and J. S. Speck, Appl. Phys. Lett. 74, 3326-3328 (1999). A. Munkholm, G. B. Stephenson, J. A. Eastman, C. Thompson, P. Fini, J. S. Speck, P. H. Fuoss, O. Auciello, and S. P. DenBaars, Phys. Rev. Lett., 83, (1999).

27. J. P. Kutter, R. S. Ramsey, S. C. Jacobson, and J. M. Ramsey, Journal of Microcolumn Separations 10, 313 (1998).

28. M. F. Crommie, D. P. Lutz, D. M. Eigler, Science 262, 218-220 (1993).

29. T. A. Jung, R. R. Schlittler, J. K. Gimzewski, H. Tang, C. Joachim, Science 271, 181-184 (1996).

30. W. W. Pai, Z. Zhang, J. Zhang, J. F. Wendelken, Surface Science 393, L106-L112 (1997).

31. B. Lai et al., Rev. Sci. Inst. 66, 2287 (1995).

32. Z. Chen et al., J. Vac. Sci. \& Tech. 15, 2522 ., (1997).

33. M. Yoon, B. C. Larson, J. Z. Tischler, J.-S. Chung, G. E. Ice, T. E. Haynes, and P. Zschack, Bulletin of the Am. Phys. Soc. 44, 1045 (1999).

34. J. Z. Tischler, M. Yoon, B. C. Larson, D. H. Lowndes, G. Eres, and P. Zschack, Bulletin of the Am. Phys. Soc. 44, 1210 (1999).

35. L. Huang, S. J. Chey, and J. H. Weaver, Phys. Rev. Lett. 80, 4095-4098 (1998),.

36. J. M. Moison, App. Phys. Lett. 64, 196-198 (1994).

37. A. Noy, D. V. Vezenov, C. M. Lieber, "Chemical force microscopy," Ann. Rev. Materials Science 27, 381-421 (1997).

38. Diode Laser Arrays, D. Botez and D. R. Scifres ed., Cambridge University Press, 1994.

39. "Towards a New Digital communication Technology Based on Nonlinear Dynamics and Chaos," Strategic Assessment Report, U.S. Army Research Office, Durham, NC, November 1996.

40. G. D. VanWiggen, R. Roy, “Communications with Chaotic Lasers," Science 279, 1198 (1998). 
41. Y. Braiman, V. Protopopescu, J. Barhen, "Chaotic Communication with Nonlinear Devices," Oak Ridge National Laboratory (in preparation, February 1999).

42. L.M. Pecora and T.L. Caroll, "Synchronization of Chaotic Systems," Phys. Rev. Lett. 64, 821 (1990).

43. H.D. Abarbanel and M. Kennel, "Synchronizing High-Dimensional Chaotic Optical Ring Dynamics," Phys. Rev. Lett. 80, 3153 (1998).

44. K.M. Cuomo and A.V. Oppenheim, "Circuit Implementation of Synchronized Chaos with Application to Communications," Phys. Rev. Lett. 71, 65 (1993).

45. G. Chen and X. Dong, From Chaos to Order, World Scientific, 1998; see also M. Lakshmanan and K. Murali, Chaos in Nonlinear Oscillators, Controlling and Synchronization, World Scientific, 1996.

46. J. F. Lindner et al, "Noise Enhanced Propagation," Phys. Rev. Lett. 23, 5048 (1998).

47. Y. Braiman et al, "Taming Spatiotemporal Chaos with Disorder," Nature 378, 465 (1995).

48. Y. Braiman et al, "Disorder-Enhanced Synchronization," Physics Letters A 206, 54 (1995).

49. E. Rodrigues et al., "Perception's Shadow: Long-Distance Synchronization of Human Brain Activity," Nature 397, 431 (1999).

50. W. H. R. Miltner et al, "Coherence of Gamma-Band EEG Activity as a Basis for Associative Learning," Nature 397, 434 (1999). 


\section{INFRASTRUCTURE AND FACILITIES FOR NANOSCALE SCIENCE AND TECHNOLOGY}

\section{Accomplishments and Current Status}

The national laboratories are uniquely poised to impact research on nanoscopic materials, due to their large-scale and multi-user neutron and $\mathrm{x}$-ray facilities. Argonne National Laboratory is the site of the Advanced Photon Source and the Intense Pulsed Neutron Source; Brookhaven National Laboratory is the home of the National Synchrotron Light Source and the High Flux Brookhaven Reactor; Oak Ridge National Laboratory houses the High Flux Isotope Reactor and will be the location of the Spallation Neutron Source; the Los Alamos Neutron Scattering Center, LANSCE, is located at the Los Alamos National Laboratory; the Lawrence Berkeley National Laboratory is the home of the Advanced Light Source; and the Stanford Synchrotron Radiation Laboratory is situated at the Stanford Linear Accelerator Center. Stewardship of these facilities is a specific mission of the Office of Science of the Department of Energy.

The wavelength or energy range of the radiation provided by these facilities is ideally suited for the reciprocal or real space characterization of nanoscopic structures, which have spatial dimensions from tenths to thousands of nanometers. X-rays in the 7-10 keV range are a perfect match for nanomaterials structural characterization. The high flux of these sources makes possible probing either the static structure or real-time changes in nanoscale structure (as described in the highlight box on "Synchrotron X-rays" in section 9). While softer x-rays can be used to this same end, soft $\mathrm{x}$-rays also can be used for real-space imaging through $\mathrm{x}$-ray microscopy. Although at present the spatial resolution is not sufficient, progress is being made to improve it.

The ability to change $\mathrm{x}$-ray energies continuously and precisely also affords other opportunities. Anomalous scattering methods, for example, are particularly well suited to the characterization of multicomponent inorganic materials, where the scattering from nanoscopic particles embedded in a host can be determined. Materials also can be characterized spectroscopically, which leads immediately to determining the electronic structure of materials, a property that is known to undergo substantial changes as the size scale is reduced. Both hard and soft $\mathrm{x}$-rays can be used for lithography, where the short wavelengths of $\mathrm{x}$-rays extend the spatial resolution (minimum feature size) to the nanometer level. Thus, from many perspectives, the match between existing synchrotron sources and nanoscale science is ideal.

Reactor and spallation neutron sources, providing neutrons with wavelengths from one-tenth to several nanometers in size, are similarly well-matched to the nanoscale. While neutrons can be used to study nanoparticles themselves, they also can be used to study protective coatings or dispersants that are placed on the nanoparticles to prevent oxidation or to disperse the particles in a solvent or host medium. These coatings are typically organic layers anchored to the surface of the particles. Alternatively, nanoscopic particles can be grown with a micellar structure or even in dendrimers, hyperbranched organic molecules. By selective labeling of the organic with deuterium, or by the use of deuterated solvents, the contrast between nanoparticle and coating can be optimized. This provides an unparalleled means of investigating the organic 
material surrounding the nanoparticle. The use of organic materials, self-assembled organic structures and organic hybrid materials is emerging as a simple yet robust route to the production of functional nanoscopic structures. The potential impact of neutron sources for the elucidation of these structures is tremendous.

The Department of Energy also supports microcharacterization and specialized single-purpose centers, including electron microscopy centers, microanalysis centers, and surface modification and characterization centers. Electron microscopy and electron diffraction have been indispensable for the visualization of nanoparticles and for quantitatively determining the crystal structures of nanoparticles. Ordered arrays of nanostructures are well suited for investigation by electron microscopic techniques.

\section{Barriers to Using National Facilities for Nanoscale Research}

The mere existence of superb facilities is not sufficient to ensure a productive research output. It is necessary to ensure that user facilities operate in a highly reliable manner, i.e. maximum availability and beam delivery. Reliability for an external "on-site" user is absolutely essential to ensure a viable research effort. For a facility to have an acceptable level of reliability, it is necessary to invest significantly in the maintenance and upgrading of the facilities. In addition, ancillary equipment at user facilities must be supported to permit full use of the facilities.

While the national facilities described above are important tools, even when flawlessly operated they cannot be used effectively without a dedicated team of scientists actively pursuing nanoscale research. Currently several national laboratories are conducting research in nanoscopic structures and are, to a greater or lesser extent, using some of the facilities available. However, the potential exists to much more effectively use these facilities by better focusing and consolidating the nanomaterials research effort. This requires an investment in nanomaterials characterization and synthesis facilities that are associated with existing major national photon and neutron sources and nanointegration facilities, and the concentration of the efforts of scientists at these laboratories into a cohesive unit. This can be accomplished by establishing Centers for Nanomaterials Research in the national laboratories in order to take full advantage of the most intense sources and capabilities, as described in the Appendix. This also will permit using facilities at these Centers to promote inter-laboratory collaborations and to build on already established expertise.

\section{Future Directions}

Establishing Centers for Nanomaterials Research will require a change in the infrastructure of national laboratories that deviates somewhat from their current mode of operation. At present the scientific effort of national laboratories tends to be broken down according to academic discipline, as opposed to a focused research thrust. However, by establishing Nanomaterials Centers (similar to the NSF Materials Research Science and Engineering Centers) the expertise from a broad range of backgrounds will be tapped. Research on nanoscopic structures involves chemistry, physics, materials science, chemical engineering, electronics, computation and biology. By focusing the research efforts of scientists in these different disciplines into interdisciplinary research groups that take advantage of the facilities, synergistic interactions will develop both within the research group and with other scientists at the facilities. While Centers will focus the research effort, they also will provide the infrastructure support (support 
personnel, technical support, laboratory support, common instrumentation needs, and close association with the major photon, neutron and nanointegration facilities) in order to perform research effectively and efficiently.

The question arises: "Should the research efforts of the Centers be focused only on utilizing the synchrotron, neutron or electron microscopy resources?" Absolutely not. The Centers must be driven by the need to carry out nanoscale research and technology development of the highest quality, be this chemistry, physics, biology or engineering, and should not be dominated by one or two techniques, in support of a nanotechnology initiative that promises great economic benefits. Nonetheless, the inherently unique capabilities for nanoscale research of these major facilities are resources that must be efficiently organized and tapped. 


\section{APPENDIX: NANOMATERIALS RESEARCH CENTERS}

The Department of Energy's multi-user synchrotron $\mathrm{x}$-ray and neutron facilities are unique tools that can have an unmatched impact on our understanding of nanoscale materials and processes and on their application for nanotechnology. The wavelength or energy range of the synchrotron $\mathrm{x}$-ray and neutron beams provided by the Advanced Light Source (ALS), the Advanced Photon Source (APS) and the Spallation Neutron Source (SNS) are ideally suited for both reciprocal and real space characterization of nanoscopic materials and structures, as described in section 10. The high flux of these sources makes possible probing both static structure or time-dependent changes in structure with nanoscale resolution.

The different characteristics and interaction mechanisms of $\mathrm{x}$-ray photons or neutrons with condensed matter confer unique strengths on each for investigations of particular types of materials and phenomena. For example, synchrotron $\mathrm{x}$-rays are well-suited to study complex, multicomponent inorganic materials, e.g. nanoscopic particles embedded in a host matrix. They also permit direct spectroscopic characterization of materials, leading to the determination of nanoscale changes in electronic structure. Furthermore, $\mathrm{x}$-rays currently provide the leading method for lithographic pattern transfer with nanometer (or slightly better) resolution. Neutrons, on the other hand, can be used to study nanoparticles themselves, as well as the protective coatings or dispersants that are placed on them, with very high contrast. Because of its sensitivity to atomic motions (vibrational modes) and to low- $Z$ atoms, neutron scattering has unparalleled strength for investigating biological molecules, as well as self-assembled organic molecules or hybrid structures that provide a promising route to the production of functional nanoscopic structures. Thus, the potential impact of intense $\mathrm{x}$-ray and neutron sources on nanotechnology is enormous.

The impact of nanoscience discoveries will depend on hierarchical linking across multiple length and complexity scales. This linking from molecular interactions to nanostructures to functional systems is a fundamental challenge of the first order, both scientifically and technologically. Emerging approaches of lithography and replication extend to $100 \mathrm{~nm}$ and lower, providing the opportunity to form a seamless integration of nano- and microtechnology. Bringing together the broad range of processing tools, characterization equipment, and technical expertise needed to span these length scales is a significant challenge. National laboratories provide the ideal setting and necessary facilities to accomplish this important task.

In order to fully exploit the nation's suite of synchrotron $\mathrm{x}$-ray and neutron facilities for the development of nanotechnology, it is proposed to establish several new Nanomaterials Research Centers. These Centers would be closely associated with the principal existing $\mathrm{x}$-ray and neutron sources and facilities for nanotechnology integration. The purpose of these Centers is to enable and optimize the use of national $\mathrm{x}$-ray and neutron user facilities for the characterization of nanomaterials, the fabrication of nanostructures, and to provide for the seamless integration of nanotechnologies. 
Nanomaterials Research Centers will provide a unique environment to explore new nanoscience developments and allow students, faculty, industrial researchers, and national laboratory scientists to work together to propose, design, and assemble these materials into useful devices. The need for Nanomaterials Research Centers has recently become widely recognized. The IWGN report calls for several new national user facilities focused on nanomaterials [1], while a recent report of the National Research Council calls for the DOE to "strengthen its support for state-of-the-art instrumentation and fabrication capabilities, including Centers for instrumentation $\mathrm{R} \& \mathrm{D}$, nanofabrication, and materials synthesis and processing at national laboratories" [2].

Each new Nanomaterials Research Center will be clearly distinguished by a materials-related "primary focus" that is based both on the type of radiation available and on existing national laboratory research strengths and characterization facilities. For example, focal research areas suitable for four Nanomaterials Research Centers could be: materials derived from or inspired by Nature; hard and crystalline materials, including the structure of macromolecules; magnetic and soft materials, including polymers and ordered structures in fluids (e.g. micelles, liquid crystals, etc.); and nanotechnology integration.

The principal barriers to applying our national synchrotron $\mathrm{x}$-ray and neutron sources for nanotechnology are discussed in section 10. The overriding need is to provide ancillary facilities and an organizational infrastructure that will focus a truly national nanomaterials research effort, thereby enabling breakthrough opportunities. The importance of Centers is that they permit bringing together the research mission of national laboratories with the educational role of universities and the problem-defining capabilities of industry [3]. The resulting partnerships across disciplines and among national laboratories, universities and industry are essential to leverage resources, to strengthen the interdisciplinary approach, and to ensure a connection to technology. Centers also provide a long-term commitment to applying intellectual excellence to significant research problems and to developing the expertise of a new generation of researchers [3]. Thus, four critical needs will be addressed by establishing Nanomaterials Research Centers in conjunction with the DOE's leading national $\mathrm{x}$-ray and neutron sources and nanointegration facilities:

1. Facilities. Investment in a suite of ancillary materials characterization and synthesis facilities is needed to permit full and efficient use of national $\mathrm{x}$-ray and neutron sources for investigations of nanoscale materials and structures, by both external users and resident scientists. Close proximity of these specialized nanomaterials research facilities to the radiation sources and integration capabilities is essential in order to maximize collaborative interactions between external users and resident scientists (see below) and to fully exploit the high-flux sources.

2. Research Organization. The nanomaterials research effort of scientists at the host laboratories (and probably elsewhere) requires some reorganization and consolidation, so that they can function as a more cohesive and interactive unit. Research on nanoscopic materials and structures involves chemistry, physics, materials science, biology, chemical engineering, electrical engineering and computation. By establishing Nanomaterials Research Centers, the research efforts of scientists at the host laboratories can be focused more effectively into highly interdisciplinary Center-based research groups that will be able best to take advantage of shared facilities and synergistic interactions, both within the 
Center and with external users. As part of this reorganization the Nanomaterials Research Centers will also establish connections with existing microscopy centers and other $\mathrm{x}$-ray and neutron user facilities at each of the national laboratories, so that these also can be fully utilized for nanotechnology.

3. Support for External Users. Nanomaterials Research Centers will provide the infrastructure support (scientific collaboration, technical support personnel, laboratory support, and common instrumentation) that visiting scientists require in order to efficiently conduct high quality nanomaterials research.

4. Education. The existence of Nanomaterials Centers equipped with facilities and interdisciplinary research teams will enable educating a new generation of young scientists, who will be the first to receive the truly interdisciplinary training that is needed for nanotechnology. Such intellectual capital is probably the most important long-term investment for science and technology [4]. The Centers as described here will be wellorganized for training graduate students and postdoctoral researchers and for strong, productive collaborations with university research groups, who will be among the principal scientific users.

\section{References}

1. This report, p. 1 .

2. Condensed Matter and Materials Physics: Basic Research for Tomorrow's Technology, p. 3, National Research Council, National Academy Press, Washington, D.C., 1999.

3. Ibid, p. 25.

4. Ibid, p. 28. 


\section{Contributors}

The BES Nanoscience/Nanotechnology Committee gratefully acknowledges the contributions of the following individuals who provided written materials or advice for this report:

Sean R. Agnew, Bill Appleton, Orlando Auciello, M. D. Barnes, P. Bellon, John Blair, Lynn A. Boatner, Yehuda Braiman, John D. Budai, George W. Crabtree, Leonard C. Feldman, C. P. Flynn, David B. Geohegan, E. P. George, Elias Greenbaum, Costas Grigoropoulos, Tony E. Haynes, Joachim Heberlein, James Hickman, O. Wayne Holland, S. Honda, Joe A. Horton, M. Z.-C. Hu, David E. Jesson, David C. Joy, Alan Krauss, W.-K. Kwok, Bennett C. Larson, D. J. Larson, Konstantin Likharev, Chain T. Liu, Arun Majumdar, P. J. Maziasz, Al Meldrum, John C. Miller, Frank A, Modine, Steve J, Pennycook, George M. Pharr, Simon Phillpot, David L. Price, Vladimir Protopopescu, David B. Poker, David Pui, J. Michael Ramsey, Nageswara Rao, Linda Reichl, Jim Roberto, Marie-Louise Saboungi, Michael Simpson, S. Strieffer, Thomas Thundat, Marty Wambsganss, John Wendelken, C. W. White, Gerry Wilemski, Steve P. Withrow, Dieter Wolf, J. H. Zhu, Ray A. Zuhr, Alex Zunger.

The Committee also thanks Sandralita Lowe for her expert assistance in preparing and editing this report. 


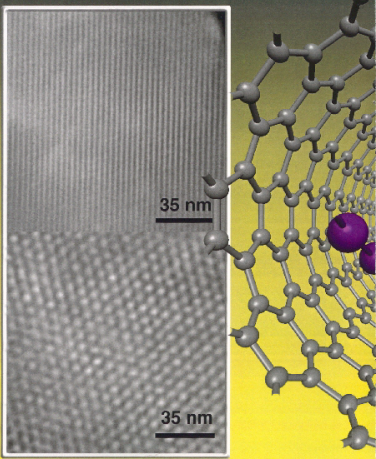

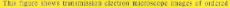

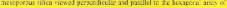

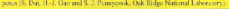

